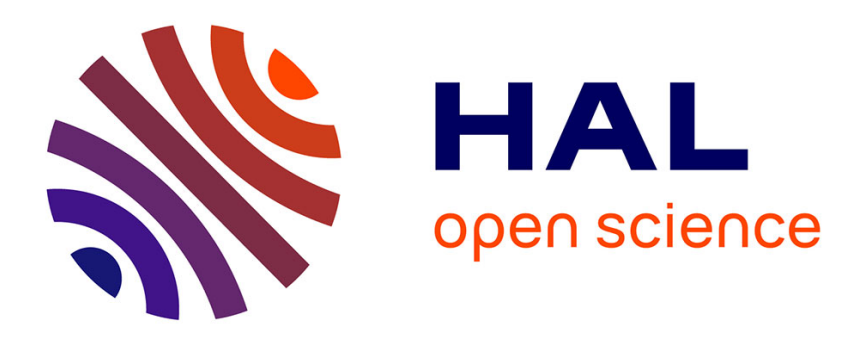

\title{
Analyse clinique, morphologique, phénotypique et moléculaire des adénocarcinomes nasosinusiens : à propos de 38 cas
}

Olivia Roels-Abramovici

\section{- To cite this version:}

Olivia Roels-Abramovici. Analyse clinique, morphologique, phénotypique et moléculaire des adénocarcinomes nasosinusiens : à propos de 38 cas. Médecine humaine et pathologie. 2012. dumas-00706064

\section{HAL Id: dumas-00706064 https://dumas.ccsd.cnrs.fr/dumas-00706064}

Submitted on 8 Jun 2012

HAL is a multi-disciplinary open access archive for the deposit and dissemination of scientific research documents, whether they are published or not. The documents may come from teaching and research institutions in France or abroad, or from public or private research centers.
L'archive ouverte pluridisciplinaire HAL, est destinée au dépôt et à la diffusion de documents scientifiques de niveau recherche, publiés ou non, émanant des établissements d'enseignement et de recherche français ou étrangers, des laboratoires publics ou privés. 


\section{THESE POUR LE DOCTORAT EN MEDECINE}

(Diplôme d'Etat)

PAR

ROELS-ABRAMOVICI Olivia

NEE LE 30 MAI 1982 A LILLE

PRESENTEE ET SOUTENUE PUBLIQUEMENT LE 12 AVRIL 2012

ANALYSE CLINIQUE, MORPHOLOGIQUE, PHENOTYPIQUE ET MOLECULAIRE DES ADENOCARCINOMES NASOSINUSIENS.

A PROPOS DE 38 CAS.

PRESIDENT DE JURY : Professeur Jean-Christophe SABOURIN DIRECTEUR DE THESE : Professeur Jean-Christophe SABOURIN 
DOYEN :

ASSESSEURS :

DOYENS HONORAIRES :

\section{Professeur Pierre FREGER}

Professeur Michel GUERBET

Professeur Benoit VEBER

Professeur Pascal JOLY

Professeur Bernard PROUST

Professeurs J. BORDE - Ph. LAURET - H. PIGUET C. THUILLEZ
MM. M-P AUGUSTIN - J.ANDRIEU-GUITRANCOURT M.BENOZIO- J.BORDE - Ph. BRASSEUR - R. COLIN E. COMOY - J. DALION -. DESHAYES - C. FESSARD - J.P FILLASTRE - P.FRIGOT -J. GARNIER - J. HEMET - B. HILLEMAND - G. HUMBERT - J.M. JOUANY - R. LAUMONIER - Ph. LAURET - M. LE FUR - J.P. LEMERCIER - J.P LEMOINE - Mle MAGARD - MM. B. MAITROT - M. MAISONNET - F. MATRAY - P.MITROFANOFF - Mme A. M. ORECCHIONI - P. PASQUIS - H.PIGUET M.SAMSON - Mme SAMSON-DOLLFUS - J.C. SCHRUB - R.SOYER - B.TARDIF -TESTART - J.M. THOMINE - C. THUILLEZ - P.TRON - C.WINCKLER L.M.WOLF

\section{I - MEDECINE}

\section{PROFESSEURS}

M. Frédéric ANSELME

M. Bruno BACHY

M. Fabrice BAUER

Mme Soumeya BEKRI

M. Jacques BENICHOU

M. Eric BERCOFF

M. Jean-Paul BESSOU

Mme Françoise BEURET-BLANQUART

M. Guy BONMARCHAND

M. Olivier BOYER

M. Jean-François CAILLARD

M. François CARON

M. Philippe CHASSAGNE

M. Alain CRIBIER (Surnombre)

M. Antoine CUVELIER

$\begin{array}{ll}\text { HCN } & \text { Cardiologie } \\ \text { HCN } & \text { Chirurgie pédiatrique } \\ \text { HCN } & \text { Cardiologie } \\ \text { HCN } & \text { Biochimie et Biologie Moléculaire } \\ \text { HB } & \text { Biostatistiques et informatique médicale } \\ \text { HCN } & \text { Médecine interne (gériatrie) } \\ \text { CRMPR } & \text { Médecine physique et de réadaptation } \\ \text { HCN } & \text { Réanimation médicale } \\ \text { UFR } & \text { Immunologie } \\ \text { HCN } & \text { Médecine et santé au Travail } \\ \text { HCN } & \text { Maladies infectieuses et tropicales } \\ \text { HB } & \text { Médecine interne (Gériatrie) } \\ \text { HCN } & \text { Cardiologie } \\ \text { HB } & \text { Pneumologie }\end{array}$


M. Pierre CZERNICHOW

M. Jean - Nicolas DACHER

M. Stéfan DARMONI

M. Pierre DECHELOTTE

Mme Danièle DEHESDIN

M. Philippe DENIS (Surnombre)

M. Jean DOUCET

M. Bernard DUBRAY

M. Philippe DUCROTTE

M. Frank DUJARDIN

M. Fabrice DUPARC

M. Bertrand DUREUIL

Mle Hélène ELTCHANINOFF

M. Thierry FREBOURG

M. Pierre FREGER

M. Jean François GEHANNO

M. Emmanuel GERARDIN

Mme Priscille GERARDIN

M. Michel GODIN

M. Philippe GRISE

M. Didier HANNEQUIN

M. Fabrice JARDIN

M. Luc-Marie JOLY

M. Pascal JOLY

M. Jean-Marc KUHN

Mme Annie LAQUERRIERE

M. Vincent LAUDENBACH

M. Alain LAVOINNE

M. Joël LECHEVALLIER

M. Hervé LEFEBVRE

M. Xavier LE LOET

M. Eric LEREBOURS

Mle Anne-Marie LEROI

M. Hervé LEVESQUE

Mme Agnès LIARD-ZMUDA
$\mathrm{HCH}$ Epidémiologie, économie de la santé

HCN Radiologie et Imagerie Médicale

HCN Informatique Médicale/Techniques de communication

HCN Nutrition

$\mathrm{HCN}$ Oto-Rhino-Laryngologie

$\mathrm{HCN}$ Physiologie

HB Thérapeutique/Médecine - Interne - Gériatrie.

CB Radiothérapie

HCN Hépato - Gastro - Entérologie

HCN Chirurgie Orthopédique - Traumatologique

$\mathrm{HCN}$ Anatomie-Chirurgie Orthopédique et Traumatologique

$\mathrm{HCN}$ Anesthésiologie et réanimation chirurgicale

HCN Cardiologie

UFR Génétique

$\mathrm{HCN}$ Anatomie/Neurochirurgie

HCN Médecine et Santé au Travail

$\mathrm{HCN}$ Imagerie Médicale

HCN Pédopsychiatrie

HB Néphrologie

$\mathrm{HCN}$ Urologie

$\mathrm{HCN}$ Neurologie

$\mathrm{CB}$ Hématologie

HCN Médecine d'urgence

HCN Dermato - vénéréologie

HB Endocrinologie et maladies métaboliques

$\mathrm{HCN}$ Anatomie cytologie pathologiques

$\mathrm{HCN}$ Anesthésie et réanimation chirurgicale

UFR Biochimie et biologie moléculaire

$\mathrm{HCN}$ Chirurgie infantile

HB Endocrinologie et maladies métaboliques

HB Rhumatologie

HCN Nutrition

HCN Physiologie

HB Médecine interne

$\mathrm{HCN}$ Chirurgie Infantile 
M. Bertrand MACE

M. Eric MALLET (Surnombre)

M. Christophe MARGUET

Mle Isabelle MARIE

M. Jean-Paul MARIE

M. LoÏc MARPEAU

M. Stéphane MARRET

M. Pierre MICHEL

M. Francis MICHOT

M. Bruno MIHOUT

M. Pierre-Yves MILLIEZ

M. Jean-François MUIR

M. Marc MURAINE

M. Philippe MUSETTE

M. Christophe PEILLON

M. Jean-Marc PERON

M. Christian PFISTER

M. Jean-Christophe PLANTIER

M. Didier PLISSONNIER

M. Bernard PROUST

M. François PROUST

Mme Nathalie RIVES

M. Jean-Christophe RICHARD (Mise en dispo)

M. Horace ROMAN

M. Jean-Christophe SABOURIN

M. Guillaume SAVOYE

M. Michel SCOTTE

Mme Fabienne TAMION

Mle Florence THIBAUT

M. LUC THIBERVILLE

M. Christian THUILLEZ

M. Hervé TILLY

M. François TRON (Surnombre)

M. Jean-Jacques TUECH

M. Jean-Pierre VANNIER
HCN Histologie, embryologie, cytogénétique

$\mathrm{HCN}$ Pédiatrie

HCN Pédiatrie

HB Médecine Interne

HCN ORL

HCN Gynécologie - obstétrique

$\mathrm{HCN}$ Pédiatrie

HCN Hépato - Gastro - Entérologie

$\mathrm{HCN}$ Chirurgie digestive

HCN Neurologie

HCN Chirurgie plastique, reconstructrice et esthétique

HB Pneumologie

$\mathrm{HCN}$ Ophtalmologie

HCN Dermatologie - Vénéréologie

$\mathrm{HCN}$ Chirurgie générale

$\mathrm{HCN}$ Stomatologie et chirurgie maxillo-faciale

$\mathrm{HCN}$ Urologie

HCN Bactériologie - Virologie

$\mathrm{HCN}$ Chirurgie vasculaire

$\mathrm{HCN}$ Médecine légale

$\mathrm{HCN}$ Neurochirurgie

$\mathrm{HCN}$ Biologie et méd. du dévelop. et de la reprod.

$\mathrm{HCN}$ Réanimation Médicale, Médecine d'urgence

HCN Gynécologie Obstétrique

$\mathrm{HCN}$ Anatomie-Pathologie

HCN Hépato-Gastro

$\mathrm{HCN}$ Chirurgie digestive

HCN Thérapeutique

HCN Psychiatrie d'adultes

HCN Pneumologie

HB Pharmacologie

$\mathrm{CB}$ Hématologie et transfusion

UFR Immunologie

HCN Chirurgie digestive

$\mathrm{HCN}$ Pédiatrie génétique 
M. Benoît VEBER

M. Pierre VERA

M. Eric VERSPYCK

M. Olivier VITTECOQ

M. Jacques WEBER

\section{MAITRES DE CONFERENCES}

Mme Noëlle BARBIER-FREBOURG

M. Jeremy BELLIEN

Mme Carole BRASSE LAGNEL

Mme Mireille CASTANET

M. Gérard BUCHONNET

Mme Nathalie CHASTAN

Mme Sophie CLAEYSSENS

M. Moïse COEFFIER

M. Vincent COMPERE

M. Manuel ETIENNE

M. Guillaume GOURCEROL

Mme Catherine HAAS-HUBSCHER

M. Serge JACQUOT

M. Joël LADNER

M. Jean-Baptiste LATOUCHE

Mme Lucie MARECHAL-GUYANT

M. Jean-François MENARD

Mme Muriel QUILLARD

M. Vincent RICHARD

M. Francis ROUSSEL

Mme Pascale SAUGIER-VEBER

Mme Anne-Claire TOBENAS-DUJARDIN

M. Eric VERIN
HCN Anesthésiologie Réanimation chirurgicale

$\mathrm{CB} \quad$ Biophysique et traitement de l'image

HCN Gynécologie obstétrique

$\mathrm{HB}$ Rhumatologie

HCN Physiologie

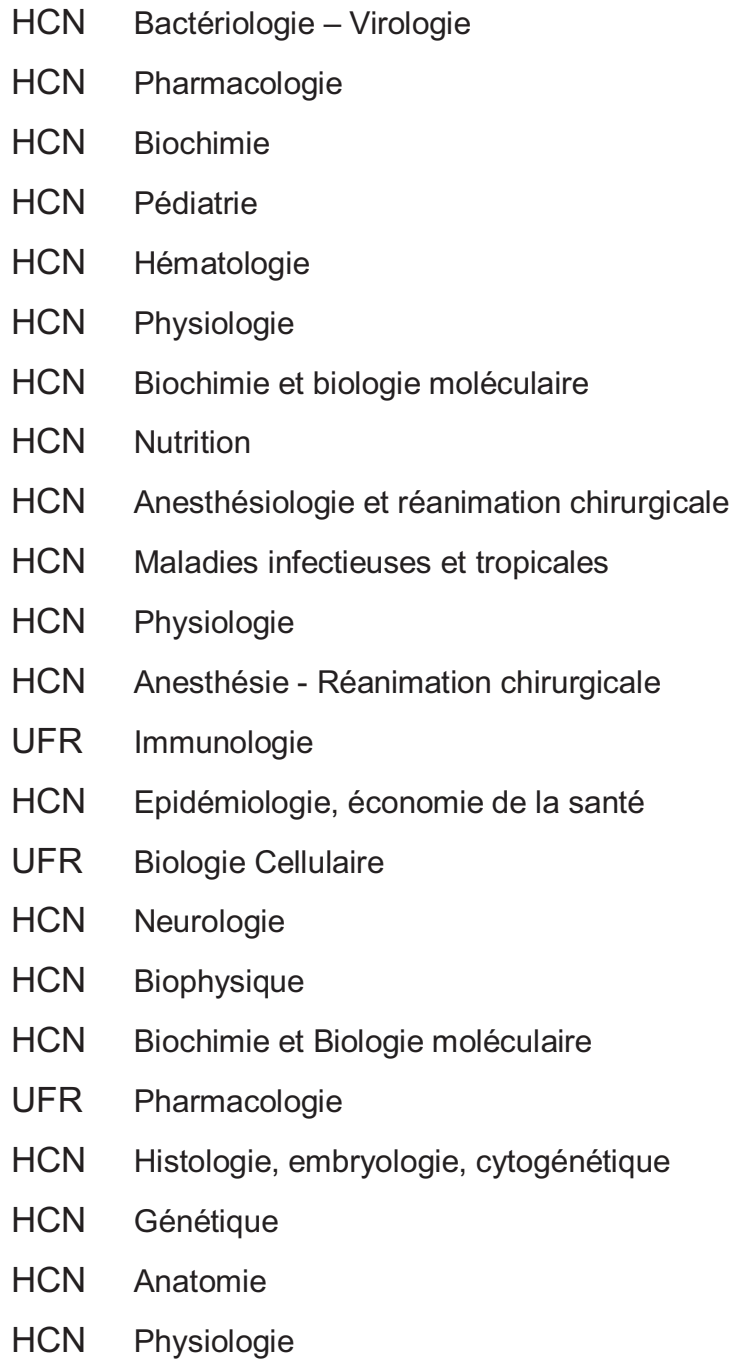

\section{MAITRE DE CONFERENCES ASSOCIE A MI-TEMPS}
M. Thierry LEQUERRE
HB Rhumatologie
M. Fabien DOGUET
HCN Chirurgie thoracique et cardio-vasculaire 


\section{PROFESSEUR AGREGE OU CERTIFIE}

Mme Dominique LANIEZ

Mme Michèle GUIGOT
UFR Anglais

UFR Sciences humaines - Techniques d'expression 


\section{PROFESSEURS}

M. Thierry BESSON

M. Jean-Jacques BONNET

M. Roland CAPRON (PU-PH)

M. Jean COSTENTIN (PU-PH)

Mme Isabelle DUBUS

M. LoÏc FAVENNEC (PU-PH)

M. Michel GUERBET

M. Olivier LAFONT

Mme Isabelle LEROUX

Mme Elisabeth SEGUIN

M. Marc VASSE (PU-PH)

$M$ Jean-Marie VAUGEOIS (Délégation CNRS)

M. Philippe VERITE

\section{MAITRES DE CONFERENCES}

Mle Cécile BARBOT

Mme Dominique BOUCHER

$M$. Frédéric BOUNOURE

Mme Martine PESTEL-CARON

M. Abdeslam CHAGRAOUI

M. Jean CHASTANG

Mme Marie Catherine CONCE-CHEMTOB

Mme Elizabeth CHOSSON

Mle Cécile CORBIERE

M. Eric DITTMAR

Mme Nathalie DOURMAP

Mle Isabelle DUBUC

Mme Roseline DUCLOS

M. Abdelhakim ELOMRI

M. François ESTOUR
Chimie Thérapeutique

Pharmacologie

Biophysique

Pharmacologie

Biochimie

Parasitologie

Toxicologie

Chimie organique

Physiologie

Pharmacognosie

Hématologie

Pharmacologie

Chimie analytique
Chimie Générale et Minérale

Pharmacologie

Pharmacie Galénique

Microbiologie

Physiologie

Biomathématiques

Législation pharmaceutique et économie de la santé

Botanique

Biochimie

Biophysique

Pharmacologie

Pharmacologie

Pharmacie Galénique

Pharmacognosie

Chimie Organique 
M. Gilles GARGALA (MCU-PH)

Mme Najla GHARBI

Mle Marie-Laure GROULT

M. Hervé HUE

Mme Hong LU

Mme Sabine MENAGER

Mme Christelle MONTEIL

M. Paul MULDER

M. Mohamed SKIBA

Mme Malika SKIBA

Mme Christine THARASSE

M. Rémi VARIN (MCU-PH)

M. Frédéric ZIEGLER

\section{PROFESSEUR ASSOCIE}

M. Jean-Pierre GOULLE

MAITRE DE CONFERENCE ASSOCIE

Mme Sandrine PANCHOU

PROFESSEUR AGREGE OU CERTIFIE

Mme Anne-Marie ANZELLOTTI

Anglais

\author{
Parasitologie \\ Chimie analytique \\ Botanique \\ Biophysique et Mathématiques \\ Biologie \\ Chimie organique \\ Toxicologie \\ Sciences du médicament \\ Pharmacie Galénique \\ Pharmacie Galénique \\ Chimie thérapeutique \\ Pharmacie Hospitalière \\ Biochimie
}

Toxicologie

Pharmacie Officinale

\title{
ATTACHE TEMPORAIRE D'ENSEIGNEMENT ET DE RECHERCHE
}
M. Bérénice COQUEREL
Chimie Analytique
M. Johann PELTIER
Microbiologie

CHEF DES SERVICES ADMINISTRATIFS : Mme Véronique DELAFONTAINE

HCN - Hôpital Charles Nicolle

HB - Hôpital de BOIS GUILLAUME

CB - Centre HENRI BECQUEREL

CHS - Centre Hospitalier Spécialisé du Rouvray

CRMPR - Centre Régional de Médecine Physique et de Réadaptation 


\section{III - MEDECINE GENERALE}

\section{PROFESSEURS}

M. Jean-Loup HERMIL UFR Médecine générale

\section{PROFESSEURS ASSOCIES A MI-TEMPS :}
M. Pierre FAINSILBER
UFR Médecine générale
M. Alain MERCIER
UFR Médecine générale
M. Philippe NGUYEN THANH
UFR Médecine générale

MAITRE DE CONFERENCES ASSOCIE A MI-TEMPS :

$\begin{array}{lcc}\text { M Emmanuel LEFEBVRE } & \text { UFR } & \text { Médecine générale } \\ \text { Mme Elisabeth MAUVIARD } & \text { UFR } & \text { Médecine générale } \\ \text { Mme Marie Thérèse THUEUX } & \text { UFR } & \text { Médecine générale }\end{array}$


Melle Cécile BARBOT

M. Thierry BESSON

M. Roland CAPRON

$M$ Jean CHASTANG

Mme Marie-Catherine CONCE-CHEMTOB

Mle Elisabeth CHOSSON

M. Jean COSTENTIN

Mme Isabelle DUBUS

M. LoÏc FAVENNEC

M. Michel GUERBET

M. Olivier LAFONT

M. Jean-Louis PONS

Mme Elisabeth SEGUIN

M. Mohamed SKIBA

M. Marc VASSE

M. Philippe VERITE
Chimie Générale et Minérale

Chimie thérapeutique

Biophysique

Mathématiques

Législation, Economie de la Santé

Botanique

Pharmacodynamie

Biochimie

Parasitologie

Toxicologie

Chimie organique

Microbiologie

Pharmacognosie

Pharmacie Galénique

Hématologie

Chimie analytique 


\section{ENSEIGNANTS MONO-APPARTENANTS}

\section{MAITRES DE CONFERENCES}

M. Sahil ADRIOUCH

Biochimie et biologie moléculaire

(Unité Inserm 905)

Mme Gaëlle BOUGEARD-DENOYELLE

Biochimie et biologie moléculaire

(Unité Inserm 614)

M. Antoine OUVRARD-PASCAUD

Physiologie (Unité Inserm 644)

\section{PROFESSEURS DES UNIVERSITES}

M. Mario TOSI

Biochimie et biologie moléculaire

(Unité Inserm 614)

M. Serguei FETISSOV

Physiologie (Groupe ADEN)

Mme Su RUAN 
Par délibération en date du 3 mars 1967, la faculté a arrêté que les opinions émises dans les dissertations qui lui seront présentées doivent être considérées comme propres à leurs auteurs et qu'elle n'entend leur donner aucune approbation ni improbation. 


\section{Monsieur le Professeur SABOURIN}

Vous aviez, dès les premières esquisses de ce travail, accepté de présider ce jury et je vous en remercie.

Je ne saurai assez vous remercier pour la qualité de vos enseignements, votre disponibilité et votre gentillesse.

C'est un honneur pour moi d'avoir été comptée parmi les internes de votre service.

Veuillez trouver, Monsieur, en ce travail, l'expression de ma plus profonde gratitude. 


\section{Madame le Professeur DEHESDIN}

C'est avec une extrême gentillesse que vous m'avez fait l'honneur d'accepter de siéger dans ce jury et je vous en remercie.

Veuillez trouver ici l'expression de mon profond respect. 


\section{Madame le Professeur GALATEAU-SALLE}

Vous avez accepté, avec beaucoup de courtoisie, de juger ce travail.

Veuillez trouver ici l'expression de mon profond respect et de ma sincère reconnaissance. 


\section{Madame le Docteur Florence Le Pessot}

C'est un honneur pour moi de te compter parmi les membres de ce jury.

Ta disponibilité, ta gentillesse et ta rigueur forcent l'admiration.

Que ce travail me permette de t'exprimer ma respectueuse reconnaissance. 
A Benichou, mon mari adoré et mon plus grand soutien. Tu es l'amour de ma vie, je suis fière d'être ta femme. Rock it all !!

A mes parents, Mireille et Luc, qui ont toujours su trouver les mots pour me réconforter et m'encourager. C'est grâce à vous si j'en suis là aujourd'hui. Je vous aime.

A Low, mon petit frère chéri, toujours dans mon cœur malgré la distance.

A Sarah, Julien et Ethanouche, mes Parisiens préférés ! Sarah, ma sœur mais aussi ma meilleure amie et confidente, je t'aime ma belle. Juju, merci de rendre ma petite sœur si heureuse et de lui avoir donné un fils si « chouchou »!

A mes grands-parents, Georgette et Maurice. Merci de nous avoir tant aimé et gâté depuis tout petit. Mes meilleurs vacances resteront celles passées à vos côtés à la piscine du Vulcano!

A ma grand-mère, Zarie. Merci pour ta présence, ton soutien et ton amour.

A Laurence, Vivi et mes cousines adorées et parfaites demoiselles d'honneurs : Julia et Anouk.

A Babeth, Valérie, Nathan, Marc et Stéphanie. Pour tous ces bons moments passés durant notre enfance.

A mes beaux-parents, Martine et Frédéric. Vous m'avez toujours accueillie avec joie. Merci pour votre soutien.

A Juliette, Sylvain et la petite Gina. Merci pour toute la fantaisie que vous mettez dans nos vies.

A Jean-Max et Julie, nos deux amis les plus fashion ! Merci pour toutes les soirées et virées effectuées en votre compagnie, vous nous manquez !!

A Yann, Helo et Fanny. En souvenir de notre road trip Argentin.

A Jenifer, Elsa, Emilie's, Tiphaine, Sonia, Fanny, Antho, Fixou... et tous les autres! Merci pour tous ces bons moments passés à Lille.

A Dalila et Solène, de véritables amies. Merci les filles pour tous ces bons moments passés à vos côtés. Dalila, merci pour ta bonne humeur si naturelle. Nos virées à Moving et nos concours de fentes vont me manquer! Solène, c'est avec joie que je te retrouve chaque jour. Je suis heureuse d'avoir passée toutes ces années avec vous. Ca risque d'être dur l'année prochaine...

A Moutaz et Emanuel. Vous égayer chaque jour le service. Moutaz, merci pour ta bonne humeur et ta gentillesse. Emanuel, tu as presque réussi à me faire aimer les autopsies !

A $M^{m e}$ Gobet et $M^{m e}$ Lemoine. Vous m'avez appris tant de choses... Merci pour tout !

A Arnaud François, Sophie Patrier, Mr Roussel, Mr Piquenot, Liana, Philippe Courville, Christelle Boivin, $\mathbf{M}^{\mathrm{me}}$ Mace, $\mathbf{M}^{\mathrm{r}}$ Landreat, $\mathbf{M}^{\mathrm{r}}$ de Kergal, $\mathbf{M}^{\mathrm{me}}$ Pellerin, $\mathbf{M}^{\mathrm{me}}$ Maillard. Merci pour votre disponibilité, votre gentillesse et votre enseignement.

A mes co-internes, Emilie, Fanny, Benjamin, Alexandre, François, Nicolas, Florent, Maxime, Hélène et Marie.

A toute l'équipe de la tumorothèque. Merci Aude pour tes conseils si précieux, ta bonne humeur et ton optimisme.

Merci à France, Jessie, Elodie, Marianne et Marion pour vos conseils et votre accueil.

Merci Elodie pour ton aide si précieuse pour de nombreuses techniques complémentaires.

A toute l'équipe d'anapath, qui rend si agréable nos journées de travail. Fabien, Annie, Martine, Rose, Michèle, Myriam, Damien, Carole, Marianne, Chantal, Marie-Noëlle, Nadine, Céline, Lucie, Alexandra, Stéphanie, Michèle, Lydie, Annie, Sylvie, Valérie, Christine, Florence, Julie, Adélaïde, Bénédicte, Céline, Dany, Sylvain...

A Alexandre Paviot et au Dr Choussy. Merci pour ce sujet passionnant. Sans vous, ce projet n'aurait jamais existé.

A Caroline Thill. Merci pour votre étude statistique si précieuse. 


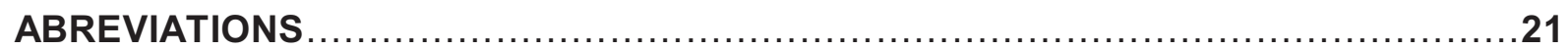

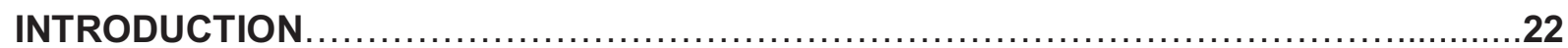

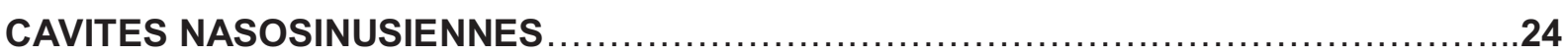

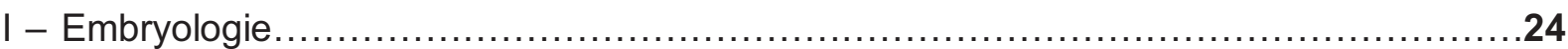

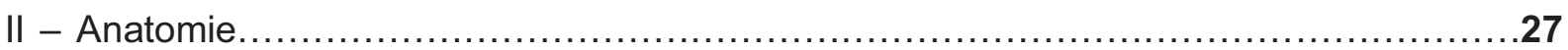

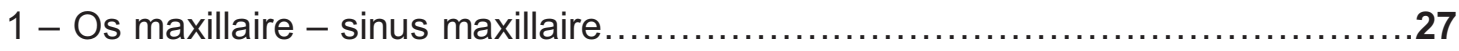

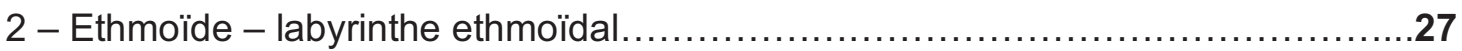

3 - Sphénoïde - sinus sphénoïdal - sinus frontal................................30

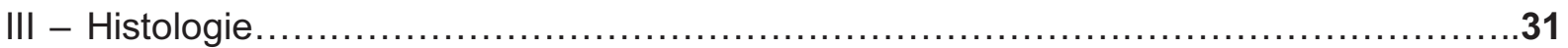

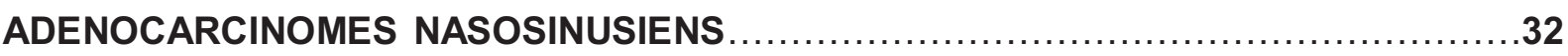

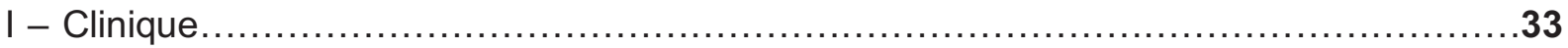

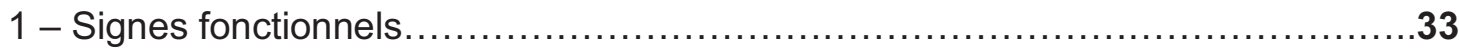

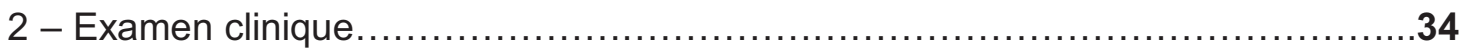

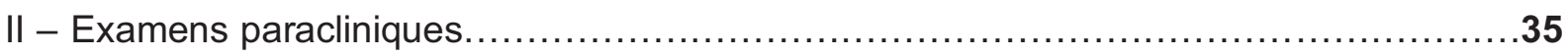

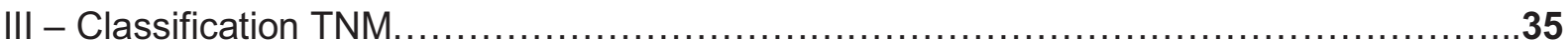

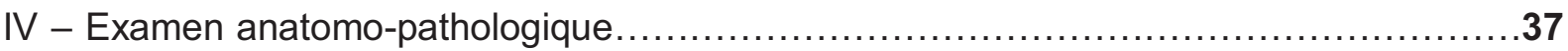

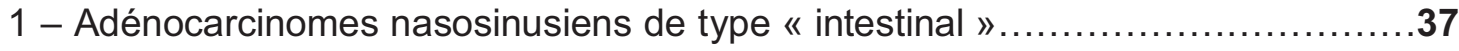

A - Epidémiologie, facteurs de risque et prévention........................37

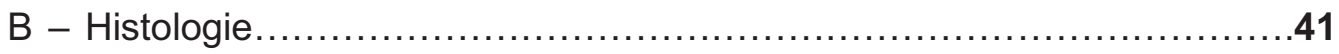

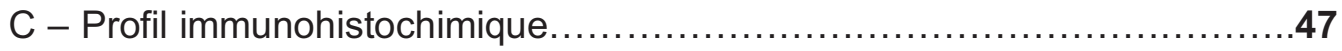

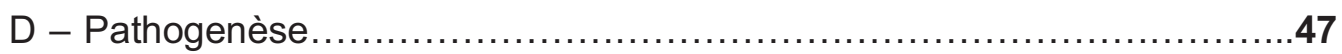

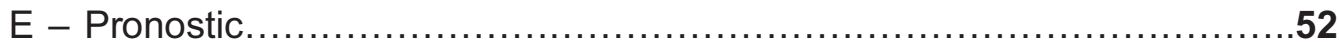

2 - Adénocarcinomes nasosinusiens de type « non intestinal $» \ldots \ldots \ldots \ldots \ldots \ldots \ldots . \ldots 53$

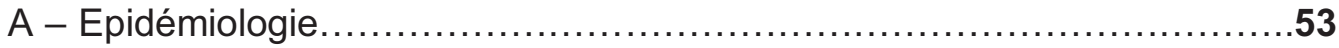

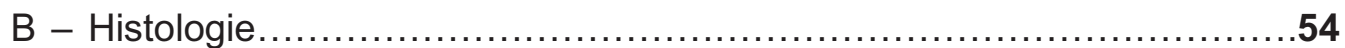

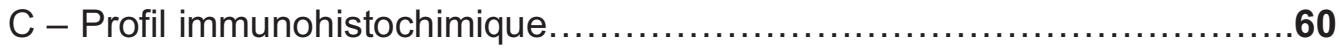

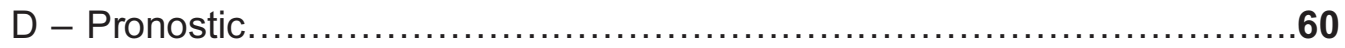

a - Adénocarcinomes nasosinusiens de bas grade .................60

b - Adénocarcinomes nasosinusiens de haut grade ...................61 
3 - Diagnostics différentiels des lésions glandulaires bien différenciées des cavités nasosinusiennes

A - Hamartome adénomatoïde respiratoire. 61

B - Papillome Schneidérien de type inversé....

C - Tumeurs développées au dépens des glandes salivaires annexées à la muqueuse nasosinusienne.

D - Métastase d'un adénocarcinome colorectal..........................66

$\mathrm{V}$ - Traitement. .67

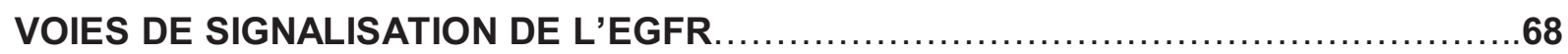

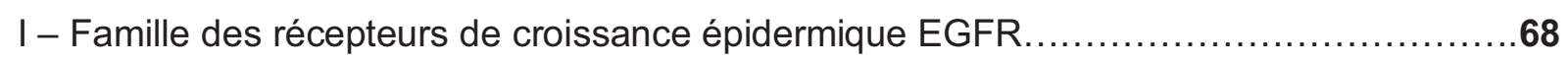

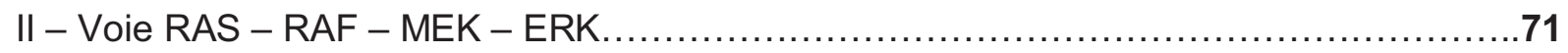

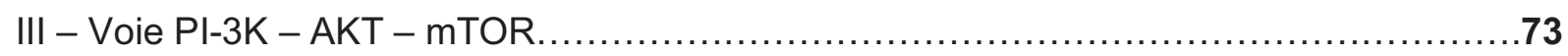

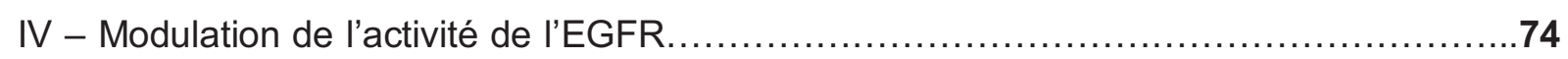

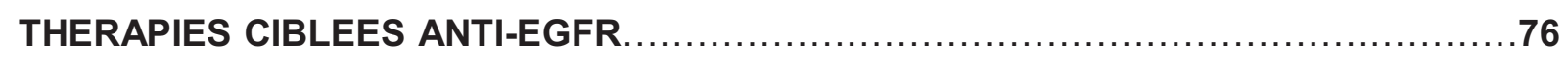

I - Les anticorps monoclonaux et les inhibiteurs de Tyrosine-Kinase ......................76

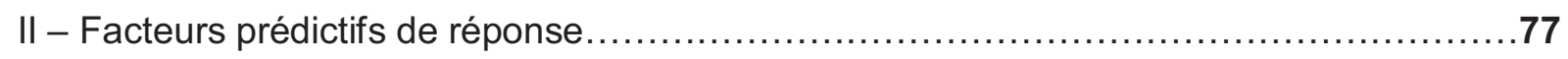

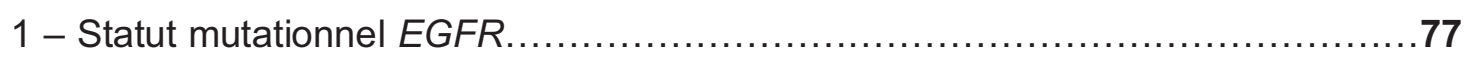

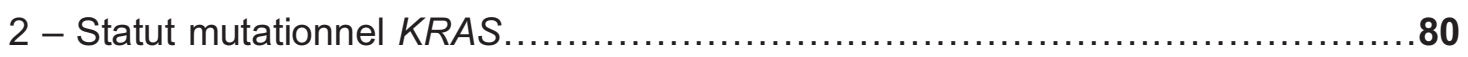

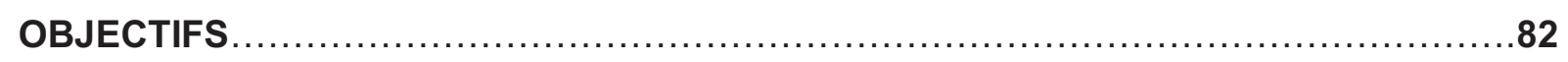

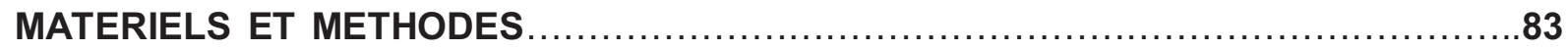

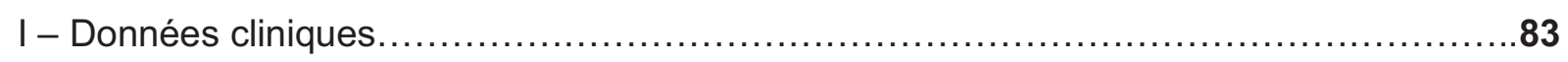

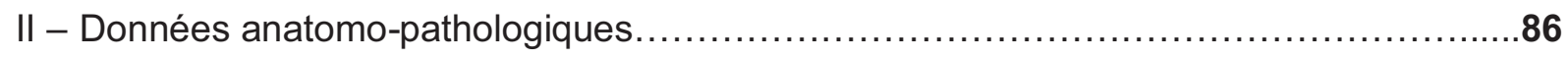

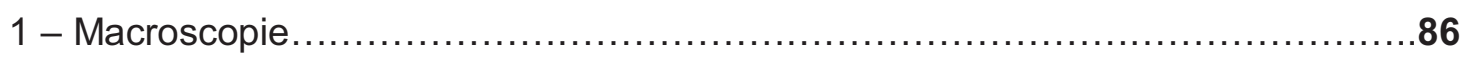

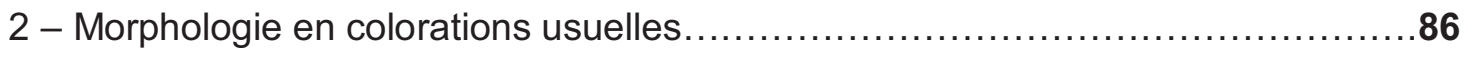

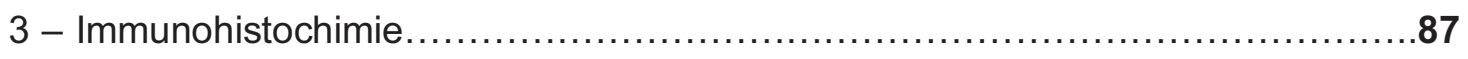

A - Le facteur de transcription CDX2 $\ldots \ldots \ldots \ldots \ldots \ldots \ldots \ldots \ldots \ldots \ldots \ldots \ldots \ldots . .87$

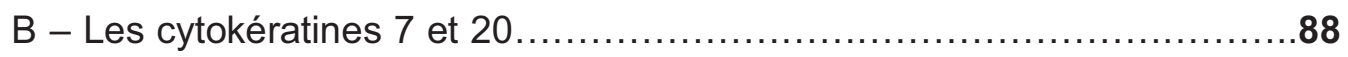

C - Le récepteur tyrosine kinase EGFR $\ldots \ldots \ldots \ldots \ldots \ldots \ldots \ldots \ldots \ldots \ldots \ldots$

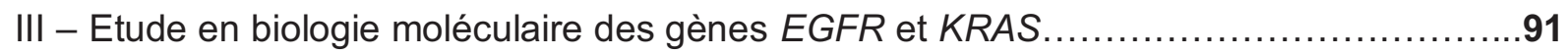

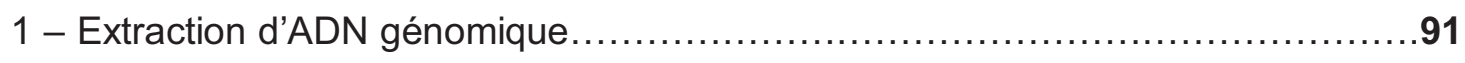

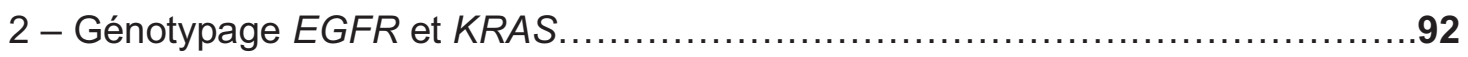

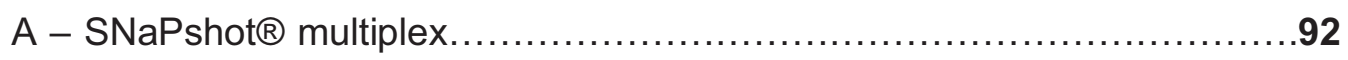




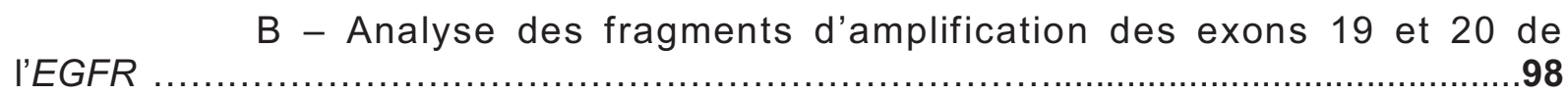

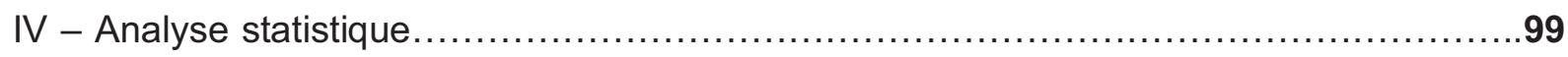

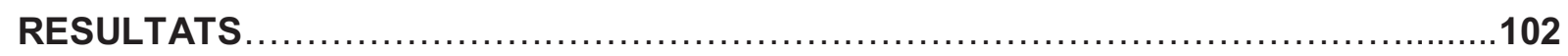

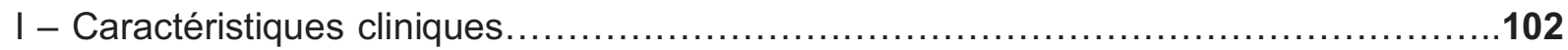

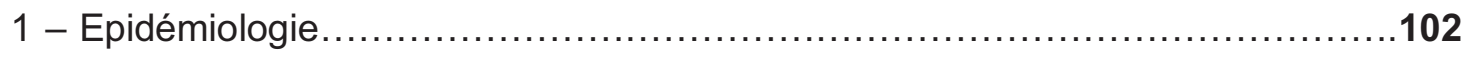

$2-$ Signes clinico-radiologiques et classification TNM.........................106

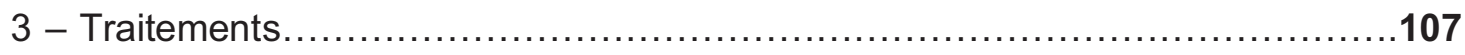

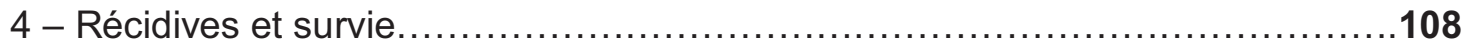

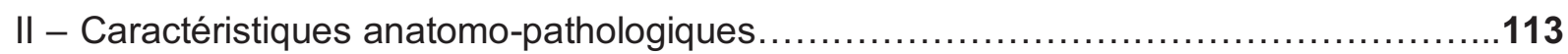

III - Caractéristiques immunohistochimiques.......................................118

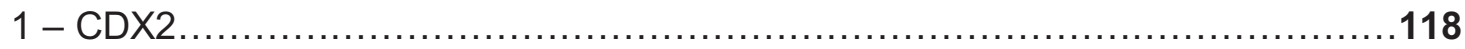

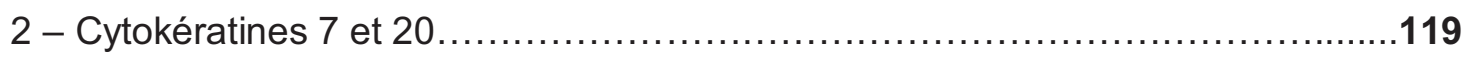

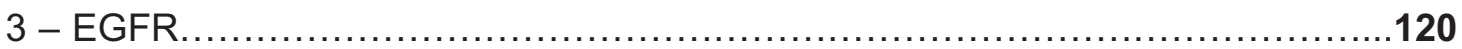

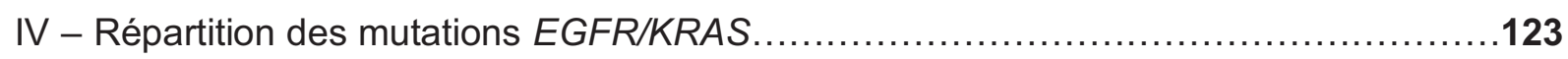

$\mathrm{V}$ - Recherche de corrélation entre le profil mutationnel KRAS et les données anatomocliniques

1 - Corrélation entre le statut mutationnel et les données cliniques. 129

2 - Corrélation entre le statut mutationnel et les données anatomopathologiques. 130

VI - Profil mutationnel tumeurs primitives/récidives locales/métastases. 131

DISCUSSION. 132

I - Analyse des caractéristiques épidémiologiques, cliniques et thérapeutiques. 132

II - Analyse des caractéristiques morphologiques..................................136

III - Analyse des caractéristiques immunohistochimiques.............................138

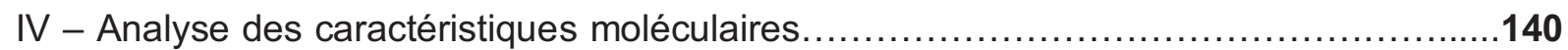

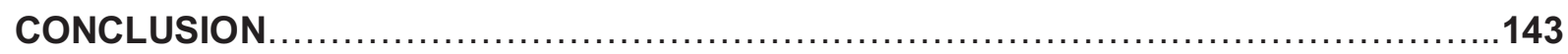

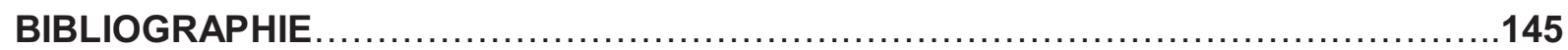

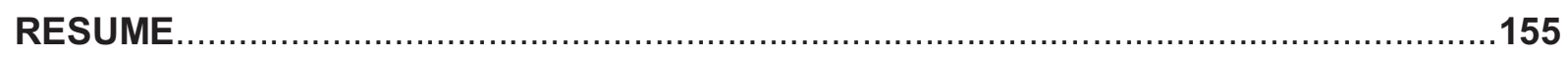




\section{ABREVIATIONS}

ADK : Adénocarcinome

CGH : Comparative Genomic Hybridization

CK : Cytokératine

EGFR = HER1 = erbB1 : Epidermal Growth Factor Receptor (Récepteur à l'EGF).

FISH : Fluorescent In Situ Hybridization

HES : Hématéine Eosine Safran

HPV : Human Papilloma Virus

IARC : International Agency for Research on Cancer

ITAC : Intestinal Type AdenoCarcinoma

MAP-K : Mitogen Activated Protein Kinase

MT : Profil muté

PAS : Periodic Acid Schiff

PCR : Polymerase Chain Reaction

PI-3K : Phosphatidylinositol 3-Kinase

TKI : Inhibiteurs de Tyrosine Kinase

WT : Wild Type (profil sauvage, non muté) 


\section{INTRODUCTION}

Les tumeurs malignes se développant dans les fosses nasales et les sinus para-nasaux sont rares. Elles représentent environ 0,2 à $0,8 \%$ de tous les cancers et 2,5 à $3 \%$ des tumeurs malignes des voies aéro-digestives supérieures.

Parmi ces tumeurs, la fréquence des adénocarcinomes est d'environ $20-25 \%$ tous sièges confondus, alors qu'elle est de $85 \%$ au niveau des sinus ethmoïdaux et affectent tout particulièrement les travailleurs du bois, figurant au tableau 47B des maladies professionnelles.

Deux principaux types histologiques ont été individualisés parmi ces adénocarcinomes ; le type "intestinal », du fait de la similitude de certaines de ces proliférations tumorales avec les adénocarcinomes intestinaux, les adénomes coliques, voire la muqueuse grêlique normale et le type « non intestinal ». Chacun de ces deux principaux types histologique est subdivisé en plusieurs sous-variétés, selon son degré de différenciation ou son aspect morphologique.

Cliniquement, ces lésions sont souvent révélées tardivement, du fait de l'apparition retardée de signes cliniques non spécifiques, dominés par des symptômes rhinologiques, suspects si unilatéraux, tels qu'une obstruction nasale, un épistaxis, une rhinorrhée ou des douleurs.

La prise en charge thérapeutique a évolué, reposant pour de nombreuses équipes sur l'association chirurgie et radiothérapie secondaire, associées parfois à une chimiothérapie .

Cependant, le pronostic de ces tumeurs est souvent défavorable, dépendant de leur contrôle locorégional, les métastases ganglionnaires ou viscérales à distance étant rares. En effet, la principale cause de décès est représentée par l'extension tumorale basi-crânienne et le taux de survie à 5 ans est estimé à $40 \%$, la plupart des décès survenant durant les 3 premières années.

De ce fait, il apparaît important de développer de nouvelles stratégies thérapeutiques. 
II est clairement établi que le développement d'un cancer est le résultat d'une combinaison entre, d'une part, l'activation de voies favorisant la prolifération cellulaire et, d'autre part, l'inhibition de signaux restreignant le potentiel prolifératif des cellules.

Parmi les voies de signalisations intracellulaires, certaines dépendent de récepteurs membranaires, appartenant à la famille erbB, comme le récepteur à l'Epidermal Growth Factor (EGF). II s'agit de récepteurs à tyrosine kinase transmembranaires qui, lorsqu'ils sont liés à leur ligand, forment un homo ou hétéro dimère conduisant à une cascade d'autophosphorylation et d'activations intracellulaires, impliquant notamment la voie de signalisation des MAP kinases (RAS-RAF-MEK-ERK) ayant pour cible des facteurs de transcription tel que C-MYC, induisant une prolifération cellulaire.

Dans de nombreux cancers, les récepteurs tyrosine kinase ou les protéines kinases en aval sont mutés ou surexprimés, ce qui active de façon non calibrée des cascades de transduction et provoque une prolifération cellulaire incontrôlée.

L'apparition de nouvelles thérapies ciblées « anti-EGFR », a largement amélioré la survie de patients atteints de pathologies carcinomateuses localement avancées ou à un stade métastatique, notamment pulmonaires et coliques.

En raison de la parenté morphologique des adénocarcinomes nasosinusiens avec ceux du tractus digestif, il pourrait être intéressant d'évaluer le potentiel d'efficacité de ces thérapies ciblées contre ces tumeurs, notamment pour des patients diagnostiqués à un stade avancé, non opérable. 
Les sinus de la face, ou sinus para nasaux constituent un ensemble de cavités aériennes développées au sein du massif facial.

Ces cavités présentent deux caractéristiques fondamentales : elles communiquent directement ou indirectement avec la cavité nasale, et sont tapissées par une même muqueuse de type respiratoire.

De ce fait, de part et d'autre de la cavité nasale, il existe des sinus maxillaires, frontaux, sphénoïdaux et des labyrinthes ethmoïdaux. Chacune de ces cavités porte le nom de la pièce osseuse au sein de laquelle elle s'est principalement développée.

\section{I - EMBRYOLOGIE}

Les gouttières olfactives apparaissent à la $5^{\circ}$ semaine chez l'embryon. Elles vont se creuser dans le massif facial pour donner la cavité nasale primitive vers la $9^{\circ}$ semaine [1].

L'hypothèse généralement admise pour la formation des sinus repose sur la muqueuse de cette cavité nasale primitive, qui, par une activité "lytique », détruirait les structures anatomiques adjacentes afin de "creuser » la cavité nasale puis les sinus para nasaux.

C'est cette origine qui explique que l'ensemble des sinus para nasaux se drainent dans la cavité nasale à partir de laquelle ils se sont développés. 
II existe plusieurs «bourgeons lytiques », ayant des trajets différents. Pour une même cavité nasale droite ou gauche, il est donc retrouvé plusieurs sous-ensembles de cavités sinusiennes qui ont un même bourgeon pour origine et qui se drainent en un même méat au sein de la cavité nasale.

Les deux sous-ensembles principaux sont:

- Sinus maxillaire, ethmoïde antérieur, sinus frontal.

- Ethmoïde postérieur, sinus sphénoïdal.

Il existe trois bourgeons principaux :

- Le bourgeon inférieur, le plus bas situé, va creuser le méat inférieur et la face inférieure du cornet inférieur.

- Le bourgeon intermédiaire, va, d'une part creuser la face supérieure du cornet inférieur et la face inférieure du cornet moyen (c'est-à-dire le méat moyen), d'autre part se diviser en :

Un bourgeon ascendant: le recessus ethmoïdo-frontal, qui creusera l'ethmoïde antérieur et le sinus frontal.

Un bourgeon descendant qui creusera le sinus maxillaire.

Le cul de sac entre ces deux bourgeons de division constitue l'infundibulum.

- Le bourgeon supéro-postérieur va creuser les cellules ethmoïdales postérieures, le sinus sphénoïdal et le méat supérieur. 


\section{Figure 1 : COUPE SAGITTALE MOYENNE DE LA VOUTE CRANIENNE ET DU MASSIF FACIAL}

(Franck H Netter, "Atlas of Human Anatomy », plate 3, 1991[2])

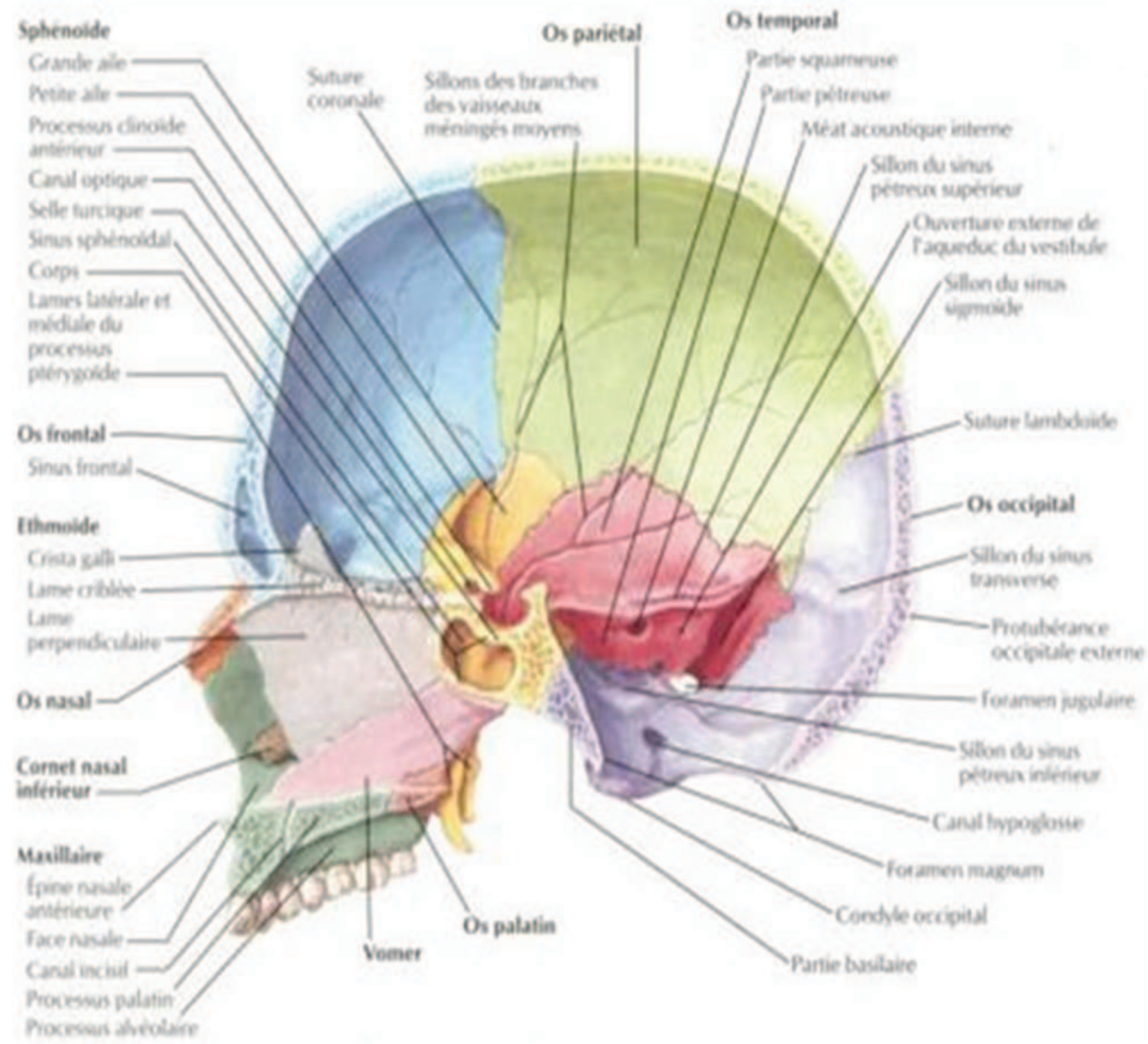




\section{II - ANATOMIE}

\section{1- Os maxillaire - sinus maxillaire}

L'os maxillaire est un os pair et symétrique. Sa morphologie globale est celle d'une pyramide dont le sommet est latéral et la base médiale. II présente un corps avec trois faces (jugale, orbitaire et infra-temporale) et quatre processus (frontal, palatin, zygomatique, alvéolaire).

La cavité creusée au sein de l'os maxillaire est le sinus maxillaire. Il est en rapport, à travers les différentes faces du corps maxillaire, avec la région jugale en avant et latéralement, avec l'orbite à travers sa face supérieure orbitaire et avec les espaces profonds de la face par son versant postérieur.

L'ostium du sinus maxillaire se situe dans la partie supérieure de la face médiale et ne s'ouvre pas directement dans la fosse nasale mais dans l'infundibulum ethmoïdal postérieur qui communique avec la cavité nasale par le méat moyen [1 - 2].

\section{2 - Ethmoïde - labyrinthe ethmoïdal}

L'ethmoïde est un os impair, médian et symétrique qui appartient à la fois à l'étage antérieur de la base du crâne et au massif facial.

II est composé de deux labyrinthes ethmoïdaux (ou masses latérales), reliés à leur partie supérieure par une lamelle osseuse horizontale nommée lame criblée. Cette dernière est percée de multiples orifices, permettant le passage des fibres olfactives de la première paire crânienne. 
A sa partie médiane, elle est divisée en deux par une lame osseuse sagittale : la lame perpendiculaire, qui participe au cloisonnement de la cavité nasale en une hémi cavité droite et gauche. En arrière et vers le bas, l'os vomer complète la lame perpendiculaire pour constituer la cloison nasale.

Les labyrinthes ethmoïdaux, présentent une morphologie quadrilatère. Ils sont creusés de cavités, appelées "cellules ethmoïdales", qui ne sont en fait pas des cellules isolées mais communiquent entre elles à la manière d'un authentique labyrinthe osseux.

Sur ses faces superficielles, dorsales et crâniales, le labyrinthe présente une particularité anatomique: les cavités les plus superficielles ne sont que des hémi cavités. Elles forment une cellule complète en se joignant avec une hémi cavité sphénoïdale (cellules dorsales) ou une hémi cavité frontale (cellules crâniales).

La face supérieure du labyrinthe ethmoïdal n'est pas directement en rapport avec la cavité crânienne, mais l'est au travers de l'os frontal.

Seule la lame criblée, qui se projette au niveau de l'incisure ethmoïdal de l'os frontal, se trouvera directement en rapport avec la cavité crânienne. Sur la partie endocrânienne de la lame criblée apparaît un élément osseux saillant antérieur qui prolonge la lame perpendiculaire, "l'apophyse Crista Galli".

La face latérale des labyrinthes éthmoïdaux est directement en rapport avec l'orbite, dont elle constitue un élément important de la paroi médiale. Cette lame osseuse particulièrement fine s'appelle « lame orbitaire » (ou "papyracée" ou "os planum").

La face médiale de chaque labyrinthe est directement en rapport avec les fosses nasales. Elle comporte d'importants reliefs constitués par des lamelles osseuses recourbées sur elles-mêmes : les cornets suprême, supérieur et moyen [1 - 2]. 
a

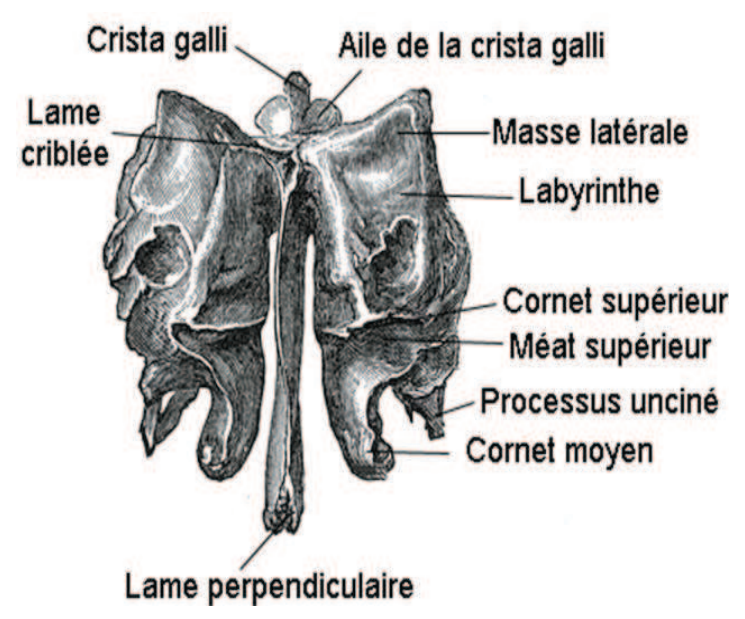

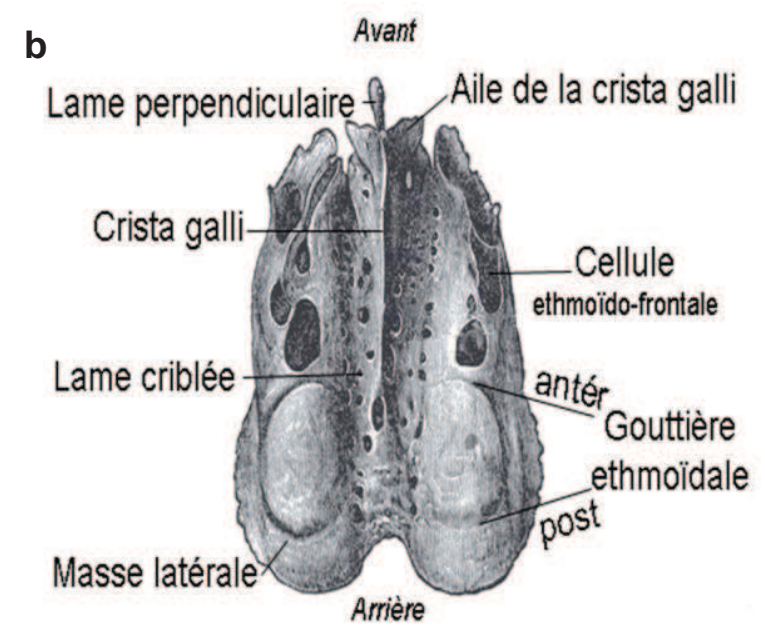

Figure $2_{A}$ : Vues postérieure (a) et supérieure (b)

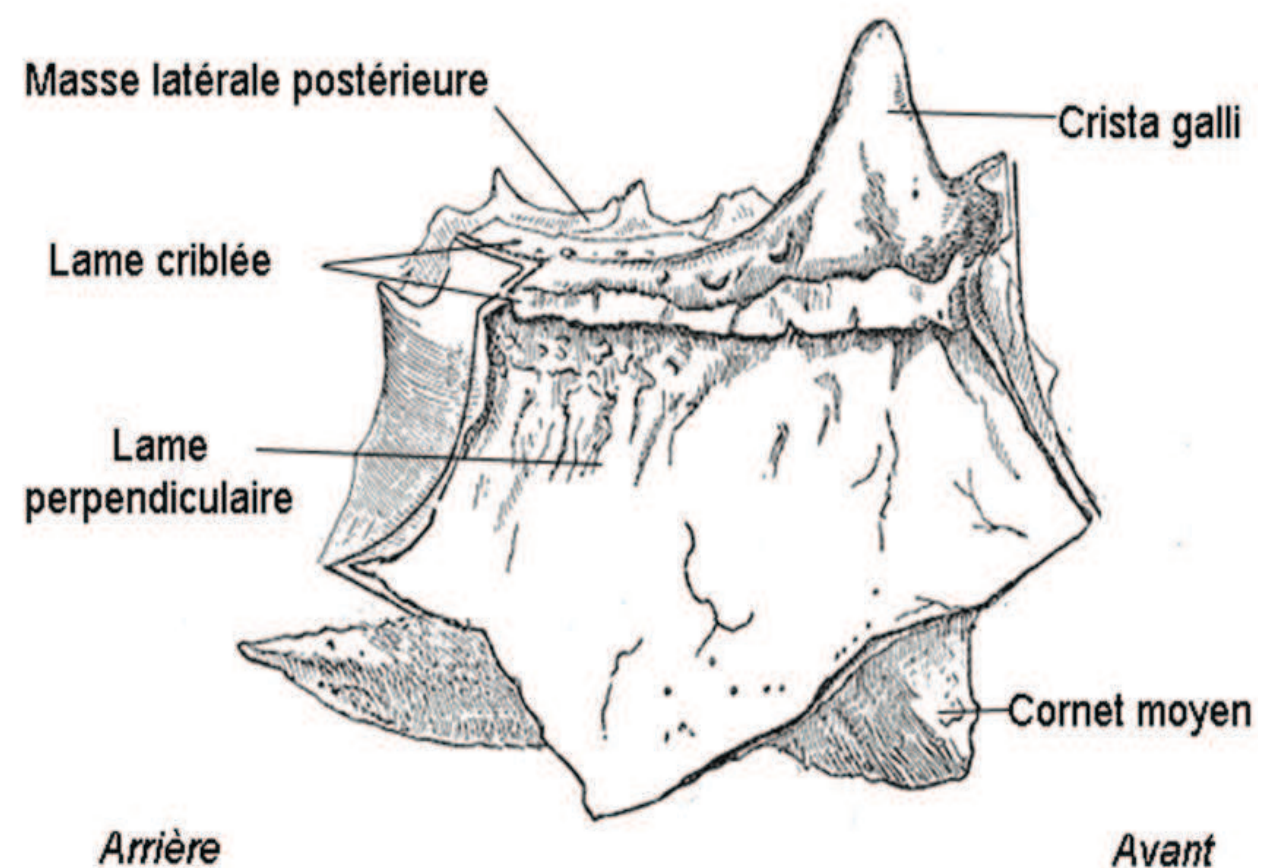

Figure $2_{B}:$ Vue latérale 


\section{3 - Sphénoïde - sinus sphénoïdal - sinus frontal}

Le sphénoïde est le siège des sinus sphénoödaux, situés en arrière des labyrinthes ethmoïdaux et qui sont les sinus para nasaux les plus postérieurs.

Le sphénoïde est un os qui participe, comme l'ethmoïde et l'os frontal, à la base du crâne.

Il est situé en arrière de l'ethmoïde. Seul le corps, partie centrale de cet os impair et médian, est intéressé par les sinus de la face.

Les rapports des sinus sphénoïdaux sont :

- En haut, le chiasma optique et la selle turcique.

- Latéralement, l'artère carotide interne et le sinus caverneux.

- En bas, l'os palatin.

Les sinus sphénoïdaux s'ouvrent dans les recessus sphéno-ethmoïdaux, bordant au dessus et en dedans le cornet supérieur.

Les sinus frontaux, de forme pyramidale, sont localisés dans la partie verticale de l'os frontal.

L'ostium des sinus frontaux s'ouvre dans la partie antérieure du méat moyen [1 - 2]. 


\section{III - $\underline{\text { HISTOLOGIE }}$}

Les cavités nasosinusiennes sont bordées par un épithélium pseudo stratifié, cilié, de type respiratoire.

Cet épithélium est essentiellement constitué de cellules cylindriques ciliées, entre lesquelles s'insèrent des cellules caliciformes mucipares.

Au sein du chorion, il est retrouvé quelques glandes séromuqueuses.

La muqueuse est séparée du périoste sous-jacent par une fine lame conjonctive [3].

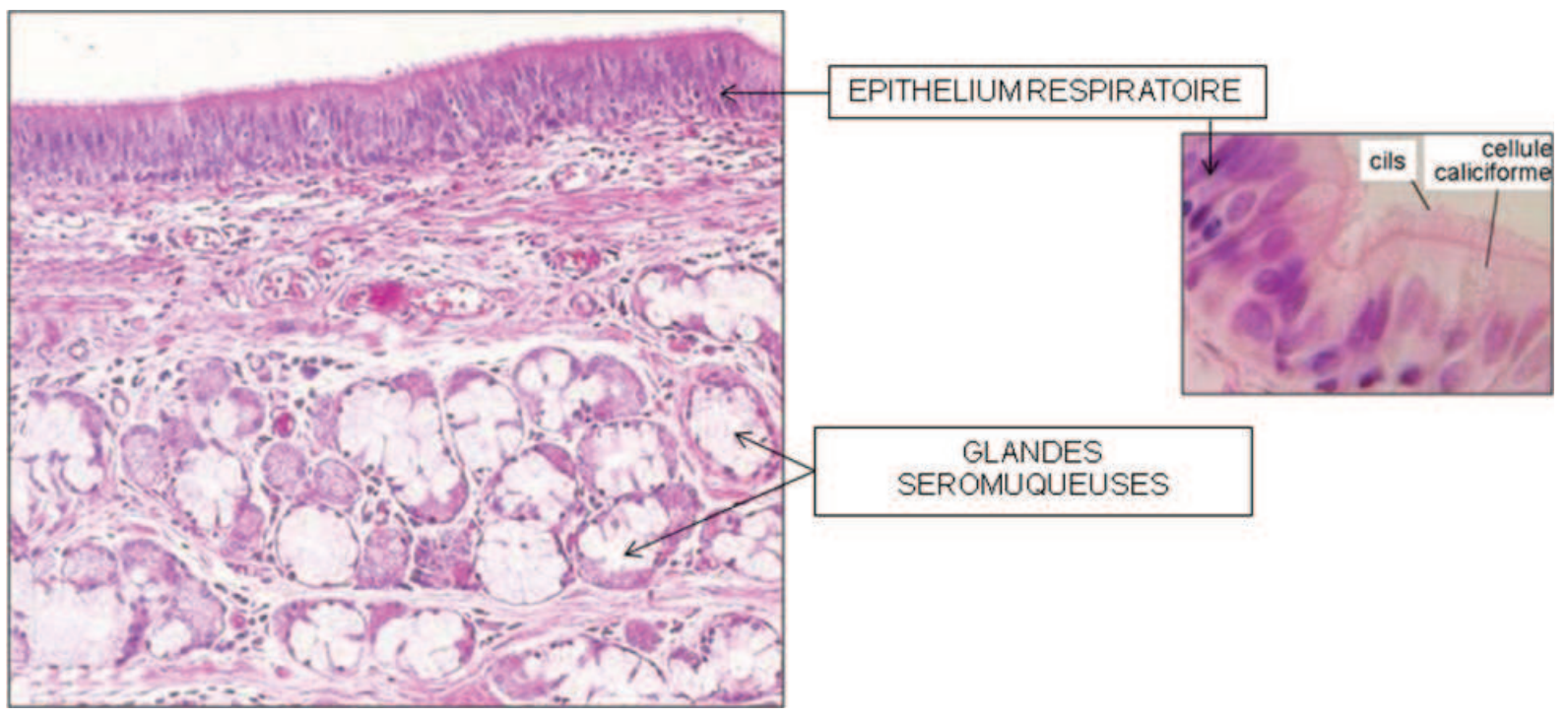

$\underline{\text { Figure } 3}$ : Muqueuse de type respiratoire 


\section{ADENOCARCINOMES NASOSINUSIENS}

Les tumeurs malignes primitives des sinus et des fosses nasales sont rares. Elles représentent environ 0,2 à $0,8 \%$ de tous les cancers et 2,5 à $3 \%$ des tumeurs malignes des voies aéro-digestives supérieures [4 - 6].

Parmi ces tumeurs, la fréquence des adénocarcinomes est d'environ $20-25 \%$ tous sièges confondus, alors qu'elle est de 80 à $85 \%$ au niveau des sinus ethmoïdaux [7].

L'incidence des adénocarcinomes nasosinusiens est relativement faible, estimée à moins de 1 cas pour 100000 habitants par an, en Europe [8].

Une très nette prédominance masculine est observée dans la majorité des séries et l'âge moyen de survenue se situe entre 40 et 70 ans, avec un pic à 60 ans [9-21].

La dernière classification OMS 2005 distingue deux types d'adénocarcinomes nasosinusiens [22] :

- Le type « intestinal », du fait de la similitude de certaines de ces proliférations tumorales avec les adénocarcinomes colorectaux, les adénomes coliques, voire la muqueuse grêlique normale.

- Le type « non intestinal ».

Chacune de ces deux principales entités histologiques est subdivisée en plusieurs sous-variétés, selon le degré de différenciation ou son aspect morphologique.

La distinction entre ces deux types d'adénocarcinomes est indispensable, puisqu'ils présentent des caractéristiques épidémiologiques et évolutives très différentes. 


\section{I - CLINIQUE}

\section{1 - Signes fonctionnels}

Les adénocarcinomes nasosinusiens sont généralement de révélation tardive, du fait de leur lente évolutivité et du caractère aspécifique des signes fonctionnels, ceux-ci se développant à un stade où la tumeur devient conséquente [9 - 21][24 29].

L'unilatéralité des symptômes devra attirer l'attention, évoquant une lésion potentiellement maligne.

Majoritairement, les signes cliniques sont dominés par une symptomatologie rhinologique : obstruction nasale et anosmie, douleur, épistaxis, rhinorrhée séreuse, purulente ou séro-sanglante.

Parfois, une symptomatologie ophtalmique peut être observée, associant une exophtalmie, une diplopie ou une baisse d'acuité visuelle, souvent tardives et devant faire suspecter une extension au sein de l'espace périorbitaire.

Des signes fonctionnels neurologiques peuvent être rapportés, dominés par des céphalées, un syndrome frontal, une détérioration intellectuelle, une hypoesthésie trijéminale ou une comitialité, suggérant une atteinte intracrânienne.

Un syndrome tumoral avec déformation de la face et une tumeur visible est également possible, traduisant souvent un envahissement massif. 


\section{2 - Examen clinique}

L'examen clinique externe est habituellement pauvre, en dehors d'une lésion évoluée avec un syndrome tumoral, une exophtalmie ou un syndrome neurologique.

Les différents paramètres suivant sont généralement explorés :

- Les fonctions oculomotrices, l'acuité visuelle, le champ visuel, la morphologie des paupières.

- Les paires crâniennes.

- Les déformations cutanées de la face.

- Les aires ganglionnaires.

L'examen rhinoscopique antérieur complété d'un examen à l'optique (souple ou rigide) après vasoconstriction permet de visualiser la tumeur lorsque celle-ci a un développement endonasale, sous forme d'un bourgeon charnu friable, saignant au contact des instruments et situé le plus souvent au niveau du méat moyen.

L'aspect peut cependant être celui d'un polype banal voire même d'une muqueuse inflammatoire.

De ce fait, toute formation tissulaire anormale des fosses nasales devra bénéficier d'une biopsie et d'un examen anatomo-pathologique.

Par ailleurs, la recherche de lésions métastatiques ganglionnaires ou viscérales à distance doit être systématique, bien qu'elles soient exceptionnelles au moment du diagnostic. En effet, les adénocarcinomes nasosinusiens présentent essentiellement une agressivité locale, avec des récidives fréquentes (50\% des cas), cause de la majorité des décès [30]. 


\section{II - EXAMENS PARACLINIQUES}

Le bilan para-clinique doit comporter au minimum [31 - 33] :

- Une tomodensitométrie (TDM) ou scanner du massif facial, permettant d'objectiver une ostéolyse et, après injection de produit de contraste, de délimiter l'extension tumorale.

- Une tomodensitométrie (TDM) ou scanner cervico-thoracique, pour évaluer l'atteinte loco-régionale et la présence d'éventuelles métastases viscérales à distance.

- Une imagerie par résonnance magnétique (IRM) de la face, permettant de définir les contours tissulaires de la tumeur, en différenciant la rétention sinusienne de l'extension tumorale. Cet examen permet également d'évaluer l'envahissement de la base du crâne, des méninges, du cerveau ou de l'orbite.

\section{III - CLASSIFICATION TNM}

La classification TNM clinico-radiologique de I'UICC 2010 (Union Internationale Contre le Cancer) [34], distingue au sein des tumeurs classées « T4 », des lésions au pronostic plus péjoratif : "T4b », contrairement à la classification de l'AJCC 1997 (American Joint Committee on Cancer) [35].

Ces deux classifications sont détaillées ci-après. 


\section{- T - TUMEUR PRIMITIVE}

- $\quad T_{1}$ : Tumeur limitée à un site de la fosse nasale ou du sinus ethmoïdal avec ou sans atteinte osseuse.

- $\quad T_{2}$ : Tumeur s'étendant à deux sites dans un seul siège ou envahissant un site voisin du complexe naso-ethmoïdal avec ou sans atteinte osseuse.

- $\quad T_{3}$ : Tumeur envahissant la paroi interne ou le plancher de l'orbite, le sinus maxillaire, le palais ou la lame criblée.

- $T_{4 a}$ : Tumeur s'étendant à l'une des structures suivantes : orbite antérieure, peau du nez ou de la joue, extension minime à l'étage antérieur de la base du crâne, apophyses ptérygoïdes, sinus sphénoïdal ou frontal.

- $T_{4 b}$ : Tumeur envahissant l'une des structures suivantes : toit de l'orbite, dure-mère, le cerveau, étage moyen de la base du crâne, les nerfs crâniens autres que le V2, le nasopharynx, le clivus.

\section{- $\quad \mathrm{N}$ - ADENOPATHIES REGIONALES}

- $\mathrm{N}_{0}$ : Pas de ganglion palpable.

- $\mathrm{N}_{1}$ : Un seul ganglion homolatéral de $3 \mathrm{~cm}$ maximum.

- $\mathrm{N}_{2}$ : a- Un seul ganglion homolatéral entre 3 et $6 \mathrm{~cm}$.

b- Plusieurs ganglions homolatéraux de moins de $6 \mathrm{~cm}$.

c- Ganglions bilatéraux ou ganglion controlatéral de moins de $6 \mathrm{~cm}$.

- $\quad \mathrm{N}_{3}$ : Ganglion de plus de $6 \mathrm{~cm}$.

- $\quad M-$ METASTASES A DISTANCE

- $M_{0}:$ Pas de métastase à distance.

- $M_{1}$ : Présence de métastase(s) à distance.

\section{Classification AJCC 1997}

$\mathrm{T}_{1}$ : Tumeur intra-ethmoïdale avec ou sans ostéolyse

$\mathrm{T}_{2}$ : Tumeur étendue aux fosses nasales

$\mathrm{T}_{3}$ : Tumeur érodant le mur orbitaire et/ou avec extension au sinus maxillaire

$T_{4}$ : Tumeur avec extension intracrânienne ou intra-orbitaire ou sphénoïdal et/ou le sinus frontal et la peau 


\section{IV - EXAMEN ANATOMOPATHOLOGIQUE}

La classification OMS 2005 [22] distingue deux principales entités histologiques : les adénocarcinomes nasosinusiens de type « intestinal » et « non intestinal ».

\section{1 - Adénocarcinomes nasosinusiens de type « intestinal » (ITAC)}

A - Epidémiologie, facteurs de risque et prévention

Par définition, les adénocarcinomes nasosinusiens de type "intestinal " sont des tumeurs dont l'aspect histologique rappelle celui des adénocarcinomes colorectaux.

La dénomination anglo-saxonne est "Intestinal Type AdenoCarcinoma " ou «ITAC ».

L'incidence est faible, estimée à moins de 1 cas par an pour 100000 habitants [36], néanmoins, ces lésions sont beaucoup plus fréquentes que les adénocarcinomes nasosinusiens de type «non intestinal ».

L'exposition aux poussières organiques de bois durs est reconnue comme facteur étiologique principal [12][25]. Les tanins, entrant dans leur composition, représenteraient le groupe le plus susceptible de jouer un rôle dans la genèse de ces lésions.

D'autres produits associés au bois peuvent être considérés comme cocarcinogènes, tels que colles et liants des contre-plaqués, ou encore peintures, solvants et vernis. 
Le lien de causalité entre les adénocarcinomes nasosinusiens de type « intestinal » et l'exposition aux poussières organiques de bois a été initialement établi en 1965, par Macbeth et Acheson [37-38]. En effet, c'est dans une étude réalisée en Angleterre, sur 68 cas de tumeurs nasosinusiennes, que Macbeth retrouve 20 cas d'adénocarcinomes dont 15 (75\%) sont survenus chez des patients travaillant dans une fabrique locale de chaises en bois. Depuis, de nombreux travaux ont prouvé le rôle favorisant de l'exposition au bois dans le développement des adénocarcinomes nasosinusiens de type intestinal, dont le risque relatif serait multiplié par 500 dans les populations exposées[39][40].

L'impact du travail du cuir dans la genèse des adénocarcinomes nasosinusiens a également été rapporté, tout particulièrement en Angleterre et en Italie, notamment dans l'industrie de la chaussure, où les sujets inhalaient des poussières contenant des tanins, provenant du tannage des cuirs par des tannates végétaux, extraits des bois feuillus [41-46].

L'effet carcinogène des tanins a été démontré expérimentalement en 1950, par l'équipe de Korpassy [47] où l'injection sous-cutanée d'acide tannique chez le rat entraînait le développement d'adénocarcinomes hépatiques, le rôle néfaste des tanins étant lié à leur capacité à entraîner la précipitation de protéines et au fait qu'il s'agisse de composés phénoliques, responsables d'irritation des muqueuses.

La durée, l'intensité et la latence de l'exposition aux poussières organiques de bois durs ou de cuirs auraient également leur importance [9-21]:

- Des excès de risque ont été rapportés dès que l'exposition était au moins égale à un an. Selon la littérature, l'exposition moyenne est comprise entre 22 et 31.5 années. De plus, le risque augmente progressivement pour devenir maximal après 30 années d'exposition. 
- Le risque serait augmenté dès que la concentration atmosphérique de poussières de bois ou de cuir est supérieure à $1 \mathrm{mg} / \mathrm{m}^{3}$.

- Des excès de risque ont été rapportés pour une latence au-delà de 20 ans, la latence moyenne étant de 40 ans.

D'autres agents tels que le nickel [48], le chrome, les poussières de charbon ou de farine, les formaldéhydes, les encres d'imprimerie contenant des tanins, le travail dans les fonderies, laminoirs et forges ainsi que l'industrie pétrolière ont été également signalés comme facteurs de risque [49]. Par ailleurs, le tabac ne serait pas un facteur carcinogène connu pour ce genre de tumeur [50].

Les adénocarcinomes nasosinusiens de type «intestinal » s'observent essentiellement chez le sujet de sexe masculin (85 à $95 \%$ selon les séries), élément probablement lié au caractère professionnel de ces tumeurs, les métiers du bois ou du cuir étant presque exclusivement exercés par des hommes.

L'âge moyen de survenue est relativement tardif, aux alentours de 60 ans, généralement dû à une durée d'exposition longue à un facteur de risque nécessaire au développement tumoral.

Ces lésions prédominent au niveau des sinus ethmoïdaux ( 80 à $85 \%$ ) et des cavités nasales [51]. Cela peut s'expliquer par le fait que la région du méat moyen et des cellules ethmoïdales moyennes correspond à la zone de turbulence du flux d'air inspiré et à la zone de drainage physiologique de la cavité nasale et des sinus paranasaux. Les particules de bois dont le diamètre est supérieur à $5 \mu$ seraient retenues par le filtre nasal et se déposeraient dans cette région.

Des cas plus sporadiques sont observés chez des sujets de sexe féminin, en particulier au niveau des sinus maxillaires (10\%) [11]. 
Devant l'important problème de santé publique posé par ce type de tumeur, la prévention est essentielle :

- Elle débute par l'information du sujet exposé au bois des risques encourus.

- Un arrêté du 28 février 1995 pris en application de l'article D461-25 du code de Sécurité Sociale prévoit la possibilité d'un examen spécialisé ORL tous les deux ans ainsi que des examens radiologiques pulmonaires et des sinus de la face.

- Une protection individuelle par port obligatoire d'un masque filtrant les petites particules est indispensable que ce soit pendant le travail professionnel ou le loisir.

- Sur le plan collectif d'autres mesures sont contrôlées par la médecine du travail, comme les aménagements des postes (hottes aspirantes, ventilations...), les mesures de concentrations pondérales en poussières de bois...

Le cancer de l'ethmoïde figure au tableau $\mathrm{n}^{\circ} 47 \mathrm{~B}$ des maladies à caractère professionnel, selon le décret 81-107 du 4 mai 1981.

Les professions concernées sont les travailleurs du bois et du cuir (fabrication et réparation des bottes et des chaussures).

II faut y associer le tableau $n^{\circ} 37$ ter concernant les travailleurs du nickel.

Le délai de prise en charge, c'est-à-dire le temps entre la fin de l'exposition et l'apparition du cancer, a été fixé par la commission des maladies professionnelles à 30 ans. 
$B-\underline{\text { Histologie }}$

De nombreuses classifications anatomo-pathologiques ont été décrites, dans le but d'établir des corrélations histopronostiques.

En l'absence de consensus, il est possible de dénombrer sur les dernières décennies presque autant de classifications que d'articles publiés sur le sujet [52-58].

Seules deux classifications sont reprises par l'OMS 2005 : celle de Kleinsasser et Schroeder [52] et celle de Barnes (figure 4) [53].

- La classification de KLEINSASSER, date de 1988 et identifie quatre sous-types d'adénocarcinomes :

- Les adénocarcinomes tubulo-papillaires à cellules cylindriques, en distinguant les formes:

Bien différenciées (grade l) : Cette sous-variété peut mimer un adénome tubulovilleux colique. La composante papillaire y est prédominante. Les axes conjonctivovasculaires des papilles sont bordés par un épithélium mono ou pseudo stratifié, composé de cellules cylindriques à plateau, auxquelles peuvent se mêler quelques cellules caliciformes ou de Paneth. Les cellules cylindriques possèdent un cytoplasme éosinophile contenant souvent au pôle apical des vacuoles de sécrétions PAS positives et en surface, des microvillosités. Leurs noyaux sont hyper chromatiques, non nucléolés, le pléomorphisme nucléaire est modéré. Au sein de ces adénocarcinomes, la sécrétion d'une substance mucineuse est habituellement peu marquée et est essentiellement intracellulaire. 
Moyennement différenciées (grade II), proche de l'adénocarcinome Lieberkühnien colique. Dans cette variante, la composante tubulaire est prédominante. Les structures glandulaires sont plus irrégulières que dans le grade I. Elles sont bordées par des cellules cuboïdales ou cylindriques atypiques, dont les noyaux souvent nucéolés, perdent leur polarité.

Peu différenciées (grade III), d'architecture trabéculaire ou solide.

- Les adénocarcinomes alvéolaires à cellules caliciformes. Cette variante tumorale prendrait naissance dans le chorion, à partir des glandes séromuqueuses et ressemble au carcinome colloïde muqueux colique. Elle est caractérisée par son importante production de mucus, pénétrée par de fins septa fibreux, responsables de l'architecture alvéolaire. La paroi de ces structures alvéolaires est bordée par une assise de cellules tumorales, qui flottent parfois au sein de plages de mucus. Les cellules tumorales sont cuboïdales, possèdent un noyau rond, hyperchrome et un cytoplasme éosinophile, contenant un produit de sécrétion ( = vacuole de mucus).

- Les adénocarcinomes à cellules en baques à chaton. Cette tumeur, identique aux carcinomes à cellules en bagues à chaton digestifs, est composée de petits amas ou d'éléments tumoraux isolés, mucosécrétants et peu atypiques, disposés au sein de plages de mucus. Le cytoplasme de ces cellules contient une vacuole de sécrétion PAS positive, correspondant à du mucus et déjetant le noyau à l'autre pôle de la cellule.

- Les adénocarcinomes transitionnels, pouvant être composés des différentes variantes décrites ci-dessus. En général, l'une des composantes est fortement majoritaire et la tumeur est rattachée à cette variante. Parfois, ces deux composantes sont représentées de façon équitable ; ces tumeurs sont dites « mixtes » ou « transitionnelles ». 
- La classification de BARNES, identifie 5 sous-types d'adénocarcinomes, quasiment superposables à ceux de la classification de Kleinsasser :

Papillaire, correspondant aux adénocarcinomes tubulo-papillaires à cellules cylindriques de grade I.

Colique, correspondant aux adénocarcinomes tubulo-papillaires à cellules cylindriques de grade II.

Solide, correspondant aux adénocarcinomes tubulo-papillaires à cellules cylindriques de grade III.

Mucineux, réunissant les adénocarcinomes alvéolaires à cellules caliciformes et à cellules en bagues à chaton.

Mixte, correspondant aux adénocarcinomes transitionnels.

Plusieurs études font état de l'aspect ultra-structural de ces tumeurs. En microscopie électronique, elles sont constituées, en proportions variables, de quatre types cellulaires: les cellules cylindriques absorbantes, les cellules caliciformes, les cellules de Paneth et les cellules argentaffines [52] [59].

La sous-variété d'adénocarcinomes nasosinusiens "intestinaux» la plus fréquemment retrouvée est de type "colique » $(40 \%)$, suivie par les sous-groupes « solide » $(20 \%)$, et « papillaire » $(18 \%)$ [22]. 
Les sous-types « mucineux » et " mixtes » sont plus rares, représentant à eux deux $22 \%$ des cas [32].

L'adénocarcinome nasosinusien « intestinal » de type «papillaire » bénéficie d'un pronostic plus favorable que les sous-types "colique » ou "solide », leur survie respective à 3 ans étant estimée à $82 \%, 54 \%$ et $36 \%$ [22].

Les adénocarcinomes "mucineux » ont un pronostic particulièrement péjoratif par rapports aux autres sous-types histologiques. L'étude de Franchi et al. [58] confirme cette hypothèse, rapportant un comportement plus agressif, ces adénocarcinomes ayant une incidence de métastase à distance de $23 \%$ et un risque de décès 11 fois plus important que les adénocarcinomes de type « papillaire ».

\section{Tableau 1}

Comparaison des classifications histologiques de Barnes et de Kleinsasser [22][51].

\begin{tabular}{|c|c|c|}
\hline BARNES & KLEINSASSER ET SCHROEDER & DESCRIPTION HISTOLOGIQUE \\
\hline PAPILLAIRE (18\%) & $\begin{array}{l}\text { TUBULO-PAPILLAIRE } \\
\text { GRADE I }\end{array}$ & $\begin{array}{c}\text { Architecture tubulo-papillaire, épithélium mono } \\
\text { ou pseudostratifié composé de cellules } \\
\text { cylindriques associées à quelques cellules à } \\
\text { mucus, polymorphisme nucléaire minime, } \\
\text { mitoses rares, atypies cytonucléaires } \\
\text { modérées. }\end{array}$ \\
\hline COLIQUE $(40 \%)$ & $\begin{array}{l}\text { TUBULO-PAPILLAIRE } \\
\text { GRADE II }\end{array}$ & $\begin{array}{l}\text { Architecture tubulo-glandulaire, épithélium } \\
\text { cylindrique ou cubique, perte de la polarité } \\
\text { nucléaire, atypies nucléaires avec nucléoles } \\
\text { proéminents, mitoses fréquentes. }\end{array}$ \\
\hline SOLIDE (20\%) & $\begin{array}{l}\text { TUBULO-PAPILLAIRE } \\
\text { GRADE III }\end{array}$ & $\begin{array}{l}\text { Architecture en nappes ou îlots cellulaires } \\
\text { avec quelques différenciations glandulaires, } \\
\text { cellules polygonales avec volumineux noyaux } \\
\text { proéminents, cytoplasme finement vacuolisés, } \\
\text { atypies sévères et mitoses fréquentes. }\end{array}$ \\
\hline MUCINEUX & $\begin{array}{c}\text { ALVEOLAIRE } \\
\text { A CELLULES EN BAGUE A CHATON }\end{array}$ & $\begin{array}{l}\text { Flaques de mucus limitées par un épithélium } \\
\text { monostratifié cubique mucosécrétant, } \\
\text { d'architecture alvéolaire. }\end{array}$ \\
\hline MIXTE & TRANSITIONNEL & $\begin{array}{l}\text { Association équilibrée de deux contingents } \\
\text { histologique }\end{array}$ \\
\hline
\end{tabular}




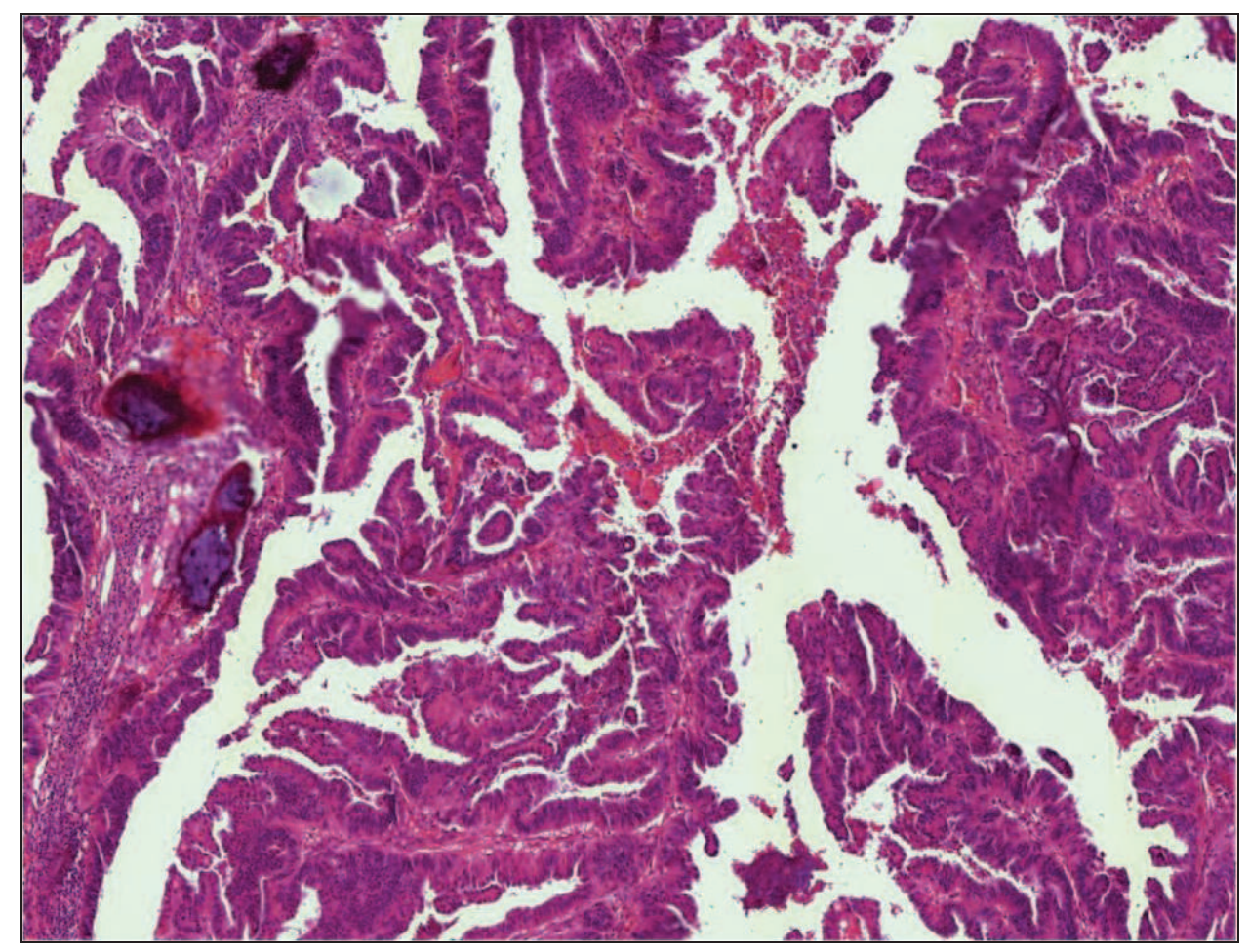

Figure $4_{A}$ : Sous-type papillaire.

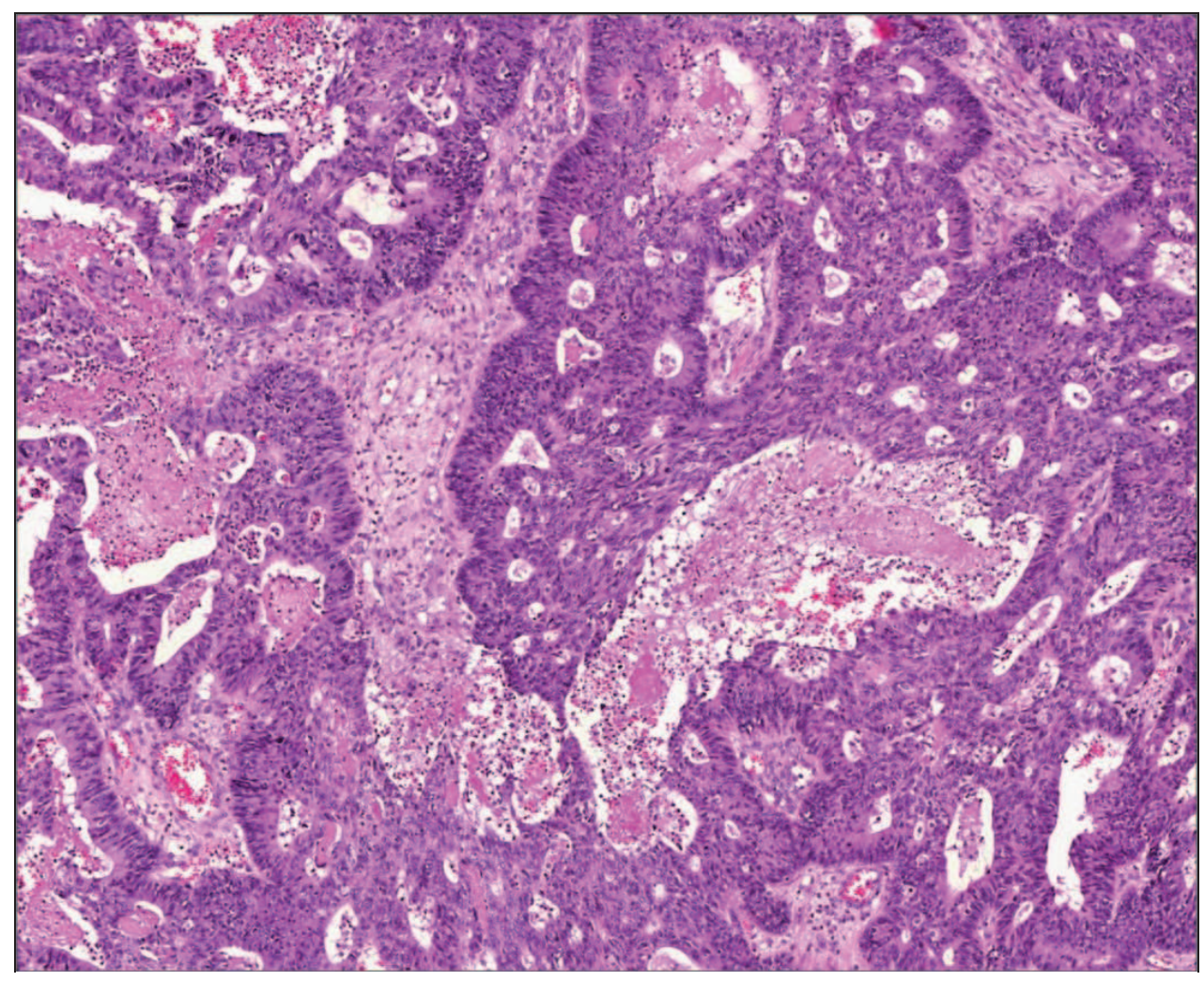

Figure $4_{\mathrm{B}}$ : Sous-type colique. 
FIGURE 4 : ADENOCARCINOMES NASOSINUSIENS DETYPE INTESTINAL

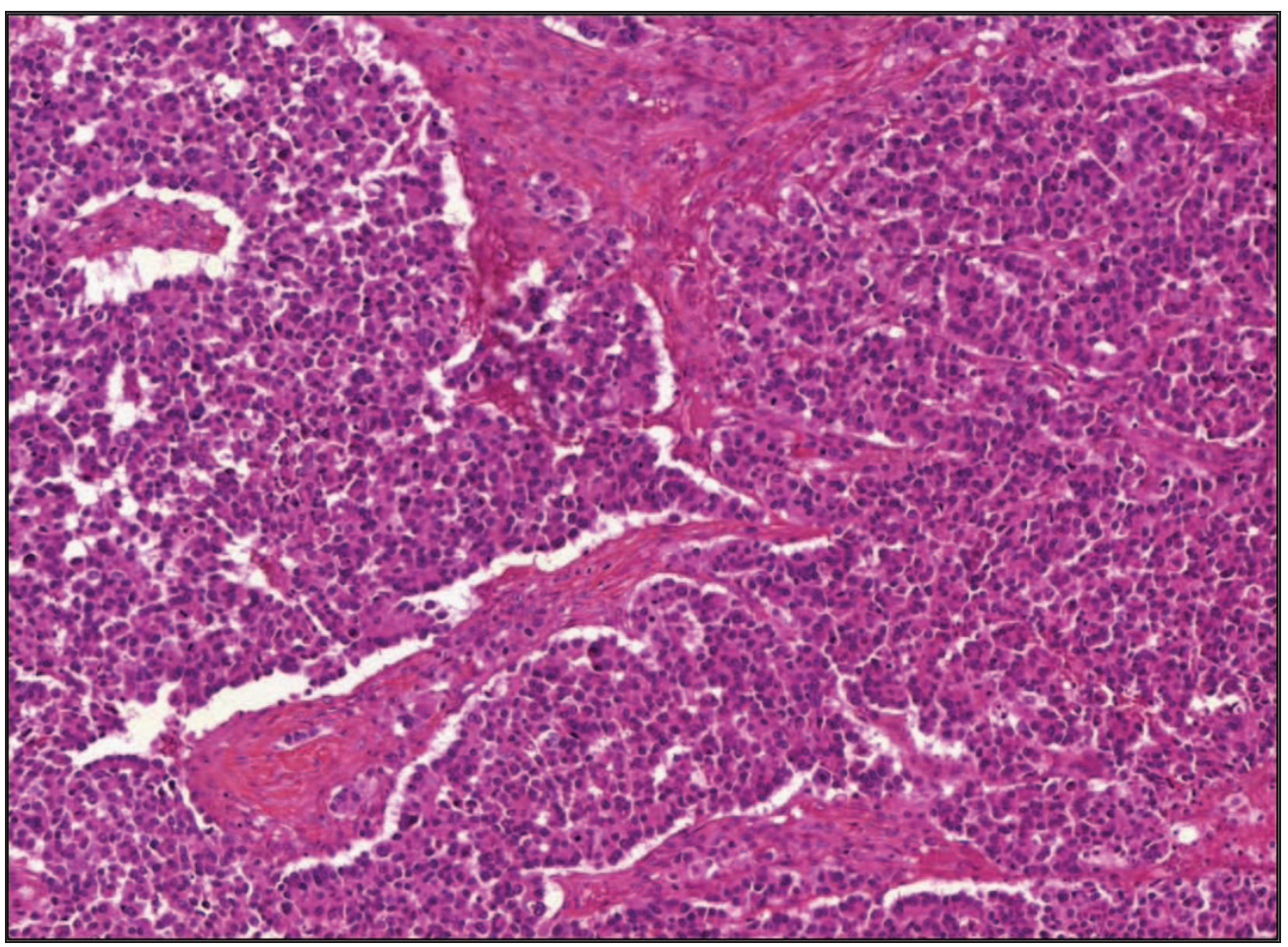

Figure $4 \mathrm{c}$ : Sous-type solide.

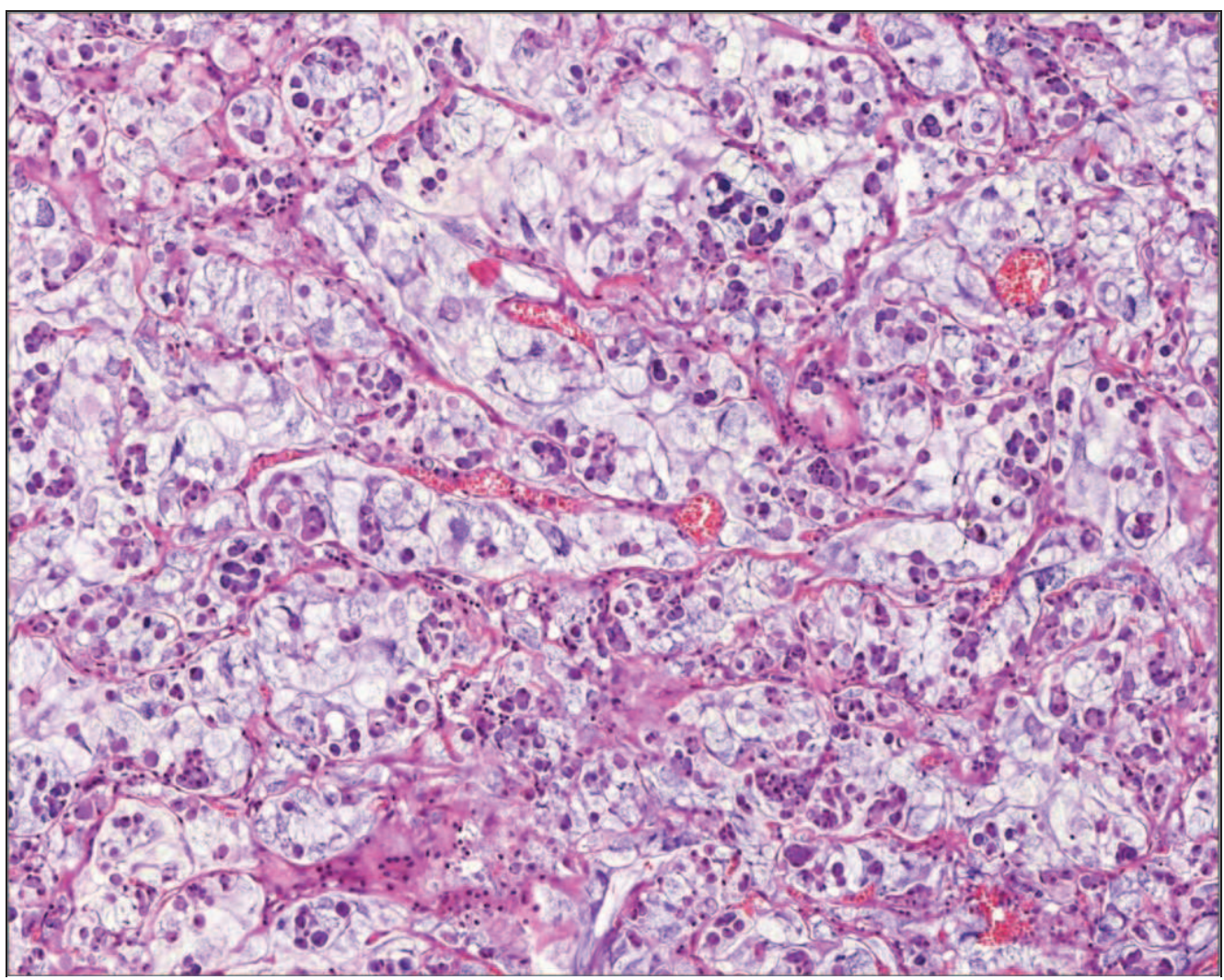

Figure $4_{D}$ : Sous-type mucineux. 
C- Profil immunohistochimique

Ces adénocarcinomes montrent généralement un profil phénotypique de type intestinal, avec positivité de la cytokératine 20 (70 à $98 \%$ selon les séries) et négativité de la cytokératine 7 (43 à 90\% selon les séries) [60-69].

Les cellules tumorales peuvent exprimer également le CDX 2 (facteur de transcription intestinal spécifique) et la villine (protéine du cytosquelette présente au niveau des microvillosités des cellules intestinales) [69].

Les adénocarcinomes nasosinusiens de type « intestinal " semblent présenter une différenciation neuroendocrine : $70 \%$ des cas dans l'étude de Bashir et al. [62] et $77 \%$ des cas dans l'étude d'Abecassis et al. [64], cette particularité ayant à priori un impact pronostic péjoratif.

$D$ - Pathogenèse

Les similitudes morphologique et immunohistochimique des adénocarcinomes nasosinusiens de type " intestinal » et des adénocarcinomes colorectaux ont poussé de nombreux auteurs à penser que ces deux processus néoplasiques partageaient les mêmes voies de tumorogenèse.

Néanmoins, leur histogenèse est sujette à des controverses.

En 1945, Järvi et al. supportait I'hypothèse d'une origine à partir de tissu gastrointestinal ectopique [70]. 
Depuis, cette hypothèse a été démentie et plusieurs théories reposant sur les données embryologiques ont été proposées.

Tôt dans le développement fœtal, la jonction entre ectoderme oronasal et endoderme pharyngé serait marquée par la membrane oropharyngée bi lamellaire qui disparaîtrait à la $4^{\text {ème }}$ semaine de gestation. La frontière entre ces deux lignées germinales serait mal définie, mais elle semblerait être immédiatement au-delà du choane postérieur, en avant de l'arche glosso-palatine. Les adénocarcinomes nasosinusiens auraient donc une origine endodermique, comme les adénocarcinomes colorectaux, à partir d'un déplacement endodermique à l'intérieur du tractus nasosinusien, durant le développement embryonnaire précoce [53].

Certains auteurs ont proposé une origine de ces tumeurs à partir de la différenciation multidirectionnelle d'une même cellule souche, qui serait une cellule cylindrique mucosécrétante apocrine, capable de donner naissance aux différentes variétés cellulaires (cellules de Paneth, endocrines, caliciformes et absorbantes) observées dans les adénocarcinomes de type intestinal. Ces mêmes auteurs ont également spéculé que cette cellule souche, par transformation directe ou par induction du mésenchyme adjacent, pourrait donner naissance à la musculaire muqueuse observée dans certaines lésions tumorales [52][71].

Par ailleurs, en fonction du type histologique, des points de départ tumoraux distincts pourraient être proposés : les tumeurs papillaires, coliques et solides pourraient se développer à partir de l'épithélium de surface et en particulier des glandes muqueuses intra épithéliales et les adénocarcinomes mucineux à partir des glandes séromuqueuses du chorion [52][53].

Certaines équipes se sont intéressées à l'aspect de la muqueuse nasosinusienne de travailleurs de bois ou de cuir, afin d'identifier des "marqueurs morphologiques" de sujets à risque. 
Plusieurs publications parlent de "lésions précancéreuses", telles qu'une métaplasie squameuse, avec ou sans dysplasie. Cependant, ces lésions sont peu spécifiques et pourraient être induites par de nombreux agents pathogènes [50][63][72-76].

L'équipe de Franchi et al. [75] a étudié la muqueuse nasosinusienne de 139 travailleurs du cuir. Une métaplasie squameuse (plus ou moins associée à des lésions dysplasiques) était notée dans $2 / 3$ des cas. Dans $20 \%$ des cas, il était également retrouvé une hyperplasie des cellules à gobelet associée à une expression de MUC-2 (apomucine glycosylée, représentant l'un des constituants majeurs du mucus), ces aspects étant liés de manière significative à une longue exposition aux poussières de cuir.

Des études ont montré que les particules de bois dont le diamètre était supérieur à $5 \mu$ seraient retenues par le filtre nasal. Leur présence serait responsable d'une métaplasie cuboïdale de l'épithélium (substitution d'un épithélium cilié par un épithélium non cilié) qui entraînerait secondairement une diminution de la clairance muco-ciliaire, aboutissant à un contact prolongé entre agent carcinogène et muqueuse [11][53].

Hermsen et al. [77] et Holmila et al. [78] ont étudié la muqueuse nasosinusienne de travailleurs $\mathrm{du}$ bois et ont également montré que l'inhalation de particules supérieures à $5 \mu$ aboutirait à un phénomène irritatif, qui stimulerait le " turn-over » cellulaire via l'activation de certaines voies de l'inflammation. De ce fait, l'exposition aux poussières de bois serait responsable d'une inflammation chronique de la muqueuse nasosinusienne avec métaplasie de l'épithélium respiratoire.

L'activation du CDX2 pourrait induire et maintenir une métaplasie intestinale avec acquisition d'un phénotype intestinal. 
D'autres évènements génétiques semblent nécessaires au développement des adénocarcinomes comme la mutation de TP53 [79-80] et la surexpression de la protéine COX2, tel que l'évoque l'étude de Kennedy et al.[65].

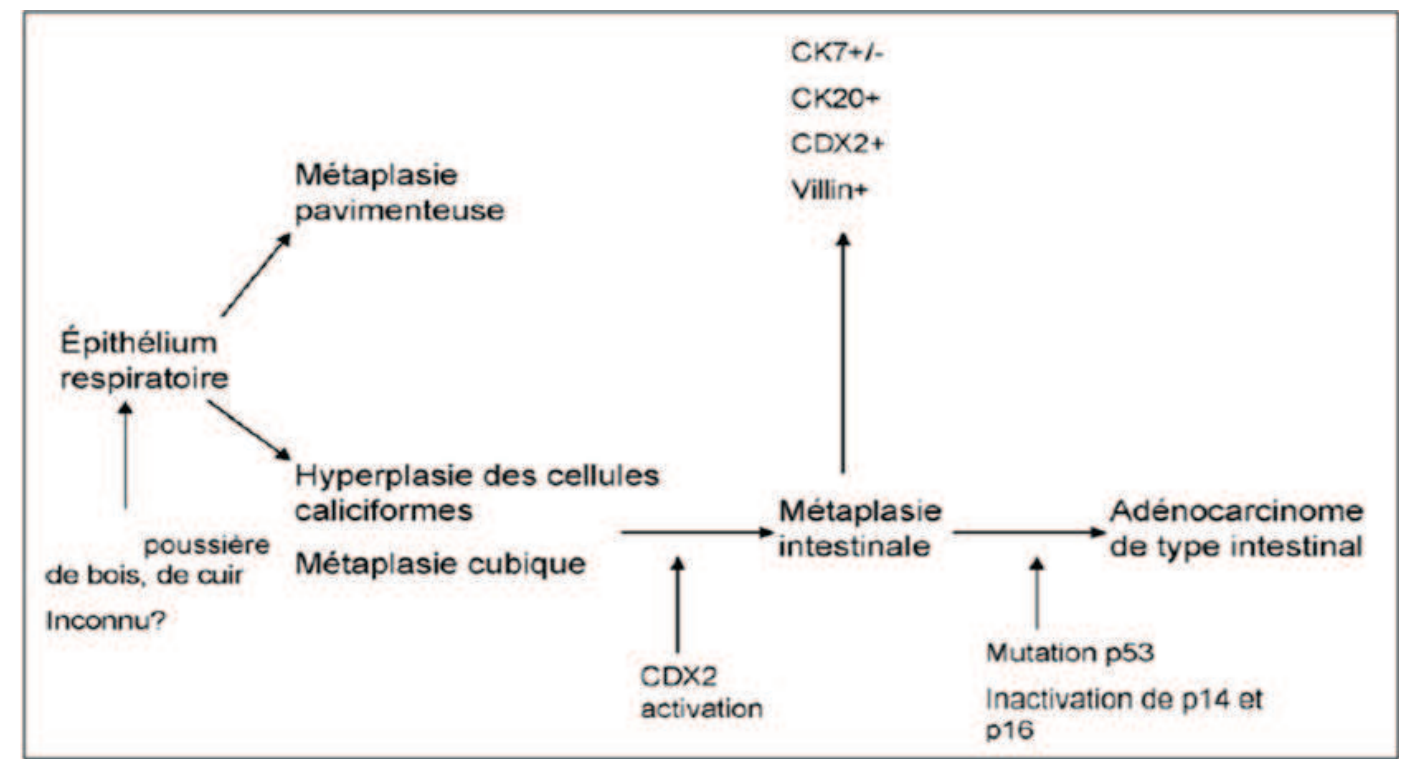

Figure 5 : Pathogenèse des adénocarcinomes de type intestinal, (Kennedy et al [65]).

Néanmoins, aucune lésion « morphologique » précancéreuse n'a été identifiée avec certitude. D'autres études ont tenté de déterminer quelles anomalies moléculaires étaient susceptibles d'être impliquées dans la pathogenèse de ces adénocarcinomes.

Valente et al. [81] a retrouvé, sur une série de 60 travailleurs du bois, une surexpression significative de la protéine p53 à la fois au niveau des territoires de métaplasie squameuse mais aussi au niveau de l'épithélium cilié et du revêtement des glandes séro-muqueuses du chorion. Des résultats similaires étaient notés dans une étude de Franchi et al. [82].

De ce fait, une surexpression de la protéine p53 en immunohistochimie pourrait aider à la distinction de sujets à risque de développer un ITAC. 
En raison de l'importante similitude morphologique entre les ITAC et les adénocarcinomes colorectaux, d'autres auteurs se sont penchées sur les gènes et les protéines connus pour être impliqués dans la croissance des tumeurs malignes digestives basses. Les principales anomalies étudiées sont:

- Les mutations du gène KRAS par des techniques de biologie moléculaire, avec des résultats très contradictoires en fonction des études.

- L'instabilité des microsatellites, observée dans les syndromes de Lynch ou HNPCC («Hereditary Non Polyposis Colorectal Cancer») et dans 5 à 10\% des formes sporadiques de cancers colorectaux. Aucune des études ne retrouve d'instabilité des microsatellites sur des séries d'adénocarcinomes nasosinusiens allant de 10 à 41 cas.

- Les altérations de la voie de signalisation Wnt, et notamment d'APC et de la $\beta$-caténine, non retrouvées dans les séries.

\section{Tableau 2 :}

Récapitulatif des études réalisées sur la carcinogenèse des ITACs.

\begin{tabular}{|c|c|c|}
\hline Auteurs & Nombre de cas & Mutations étudiées \\
\hline Wu et al. [83] & 11 cas & $\begin{array}{l}\text { KRAS : pas de mutation } \\
\text { P53: } 2 \text { cas mutés }\end{array}$ \\
\hline Saber et al. [84] & 28 cas & KRAS : 4 cas mutés \\
\hline Perez et al. [85] & 31 cas & $\begin{array}{c}\text { HRAS : } 5 \text { cas mutés } \\
\text { KRAS : pas de mutation }\end{array}$ \\
\hline Yom et al. [86] & 8 cas & $\begin{array}{c}\text { KRAS : } 2 \text { cas mutés } \\
\text { Instabilité des microsatellites : } 1 \text { cas }\end{array}$ \\
\hline Perez et al. [87] & 10 cas & Pas d'instabilité des microsatellites \\
\hline Frattini et al. [88] & 20 cas & $\begin{array}{c}\text { KRAS : } 9 \text { cas mutés } \\
\text { Instabilité des microsatellites : } 1 \text { cas }\end{array}$ \\
\hline Bornholdt et al. [89] & 58 cas & KRAS : $13 \%$ mutations \\
\hline Hermsen et al. [77] & 22 cas & $\begin{array}{c}\text { Pas d'instabilité des microsatellites } \\
\text { Pas d'anomalie chromosomique } \\
\text { spécifique }\end{array}$ \\
\hline Martinez et al. [90] & 41 cas & Pas d'instabilité des microsatellites \\
\hline Franchi et al. [91] & 62 cas & $\begin{array}{l}\text { P53 : surexpression dans } 60 \% \text { des cas } \\
\text { P16: altération dans } 60 \% \text { des cas } \\
\text { B-caténine / APC : pas d'altération }\end{array}$ \\
\hline
\end{tabular}




\section{E - Pronostic}

Les adénocarcinomes nasosinusiens de type «intestinal » présentent essentiellement une agressivité locale, avec des récidives fréquentes ( $50 \%$ des cas), cause de la majorité des décès.

Les métastases ganglionnaires sont rares (10\%). Les métastases à distance surviennent dans $20 \%$ des cas.

Le taux de survie à 5 ans, tout type histologique confondu, est d'environ $40 \%$, la plupart des décès survenant dans les 3 ans [92-93].

Aucune étude n'a réellement montré que le stade clinique pouvait avoir une valeur pronostique significative ; cela s'explique par le fait que la majorité des patients sont diagnostiqués à un stade avancé.

II semble néanmoins que les tumeurs diagnostiquées à un stade plus précoce puissent bénéficier d'un meilleur contrôle local et donc avoir un pronostic plus favorable. 


\section{2 - Adénocarcinomes nasosinusiens de type « non intestinal »}

\section{A - Epidémiologie}

Par définition, il s'agit d'adénocarcinomes primitifs du tractus nasosinusien qui n'ont pas la morphologie d'ITAC ou de tumeur développée aux dépens des glandes salivaires.

Ces tumeurs occupent l'ethmoïde dans $30 \%$ des cas, les cavités nasales dans $22 \%$ des cas, le septum nasal dans $17,5 \%$ des cas, le maxillaire dans $13 \%$ des cas et sont d'origine indéterminées dans $17,5 \%$ des cas [36].

Ces lésions surviennent majoritairement chez l'adulte, mais ont été identifiés chez des patients de 9 à 80 ans (âge moyen : 53 ans).

Aucune étude ne met en évidence de prédominance ethnique ou sexuelle et, contrairement aux ITAC, l'exposition aux poussières de bois ne constitue pas un facteur de risque.

Il n'y a pas d'association connue avec le tabac ou la consommation d'alcool.

De même, aucun facteur de risque environnemental, occupationnel ou génétique n'a été mis en évidence. 


\section{B - $\underline{\text { Histologie }}$}

Dans la classification OMS 2005 [22], deux groupes d'adénocarcinomes de type « non intestinal » sont individualisés, en fonction du degré des atypies cytonucléaires, de l'index mitotique et des remaniements nécrotiques: " l'adénocarcinome de bas grade » et «l'adénocarcinome de haut grade ».

\section{- L'adénocarcinome de bas grade :}

Ce sous-type histologique a été décrit et individualisé en 1982 par Heffner et al. [94].

Plusieurs auteurs suggèrent leur développement à partir des tubules terminaux des glandes séromuqueuses du chorion.

Dans la littérature, il est retrouvé de nombreuses descriptions histologiques, relativement comparables d'un point de vue morphologique mais répondant à des dénominations différentes : l'adénocarcinome du tubule terminal de Kleinsasser [95], l'adénocarcinome séro-muqueux de Neto et al. [96], l'adénocarcinome sinonasal tubulopapillaire de bas grade de Skalova et al. [97] et Luna [98] et l'adénocarcinome de bas grade de Heffner et al. [94] et Cathro et Mills [67].

II s'agit de tumeurs circonscrites ou infiltrantes d'architecture le plus souvent tubulopapillaire, parfois purement tubulaire ou compacte. 
Les glandes sont de petites taille, uniformes, accolées les unes aux autres de façon coalescente, sans ou avec très peu de stroma interposé entre elles. Elles sont tapissées par une simple assise de cellules non ciliées, cubiques ou cylindriques basses dotées d'un noyau rond, le plus souvent régulier, uniforme, en position basale, sans nucléole visible. Le cytoplasme est éosinophile. Les cellules ne montrent pas d'atypie cytonucléaire. Les mitoses sont rares. II n'y a pas de nécrose, pas d'engainement péri-nerveux ni d'invasion vasculaire.

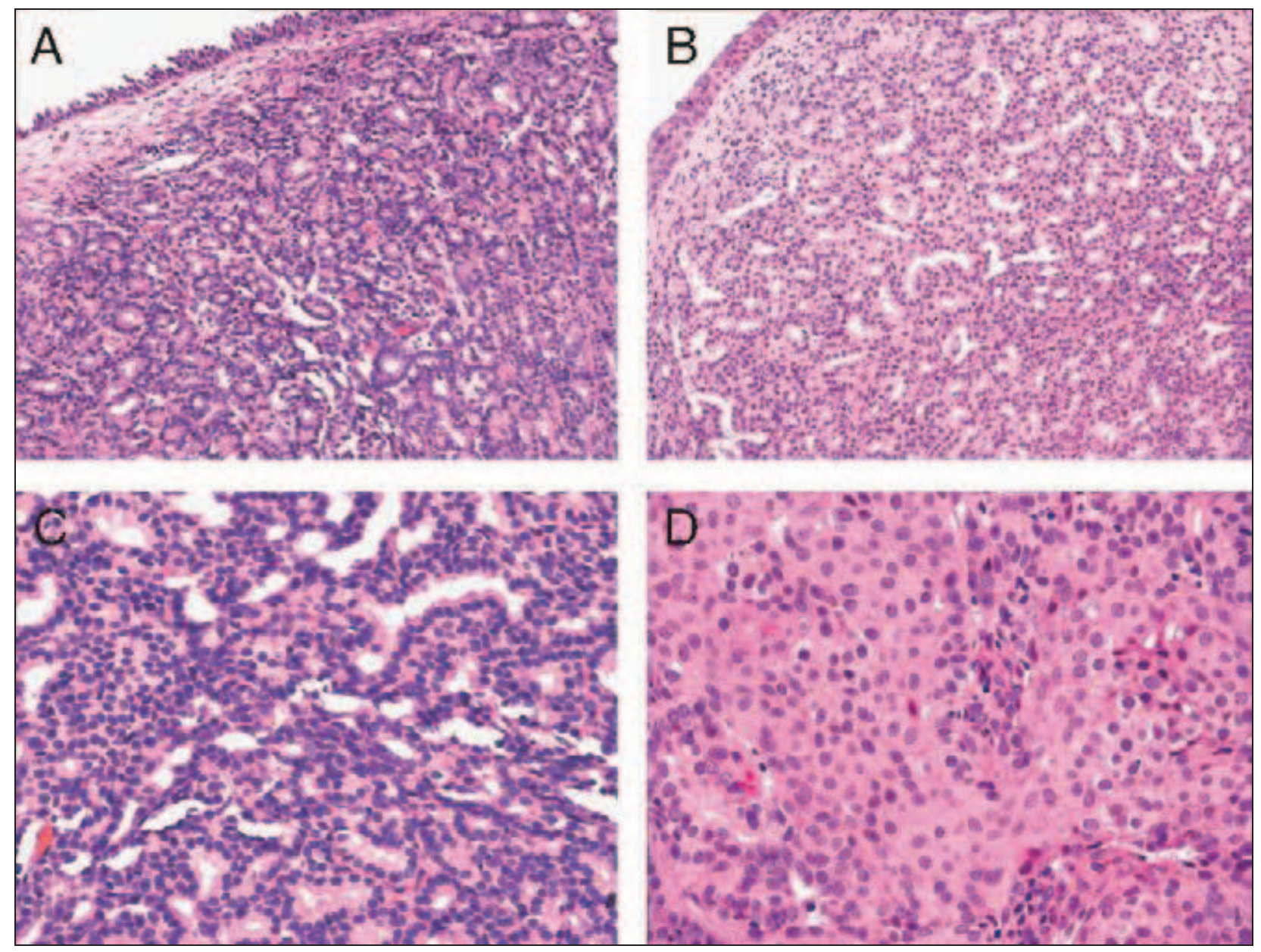

Figure 6 : Adénocarcinome nasosinusien « non intestinal de bas grade »

Architecture tubulo-papillaire $(A, B, C)$. Glandes adossées, tapissées par un épithélium uni stratifié, constitué de cellules éosinophiles non atypiques.

Une métaplasie squameuse (D) peut parfois être observée

(Jo $V$ et al.[99]). 


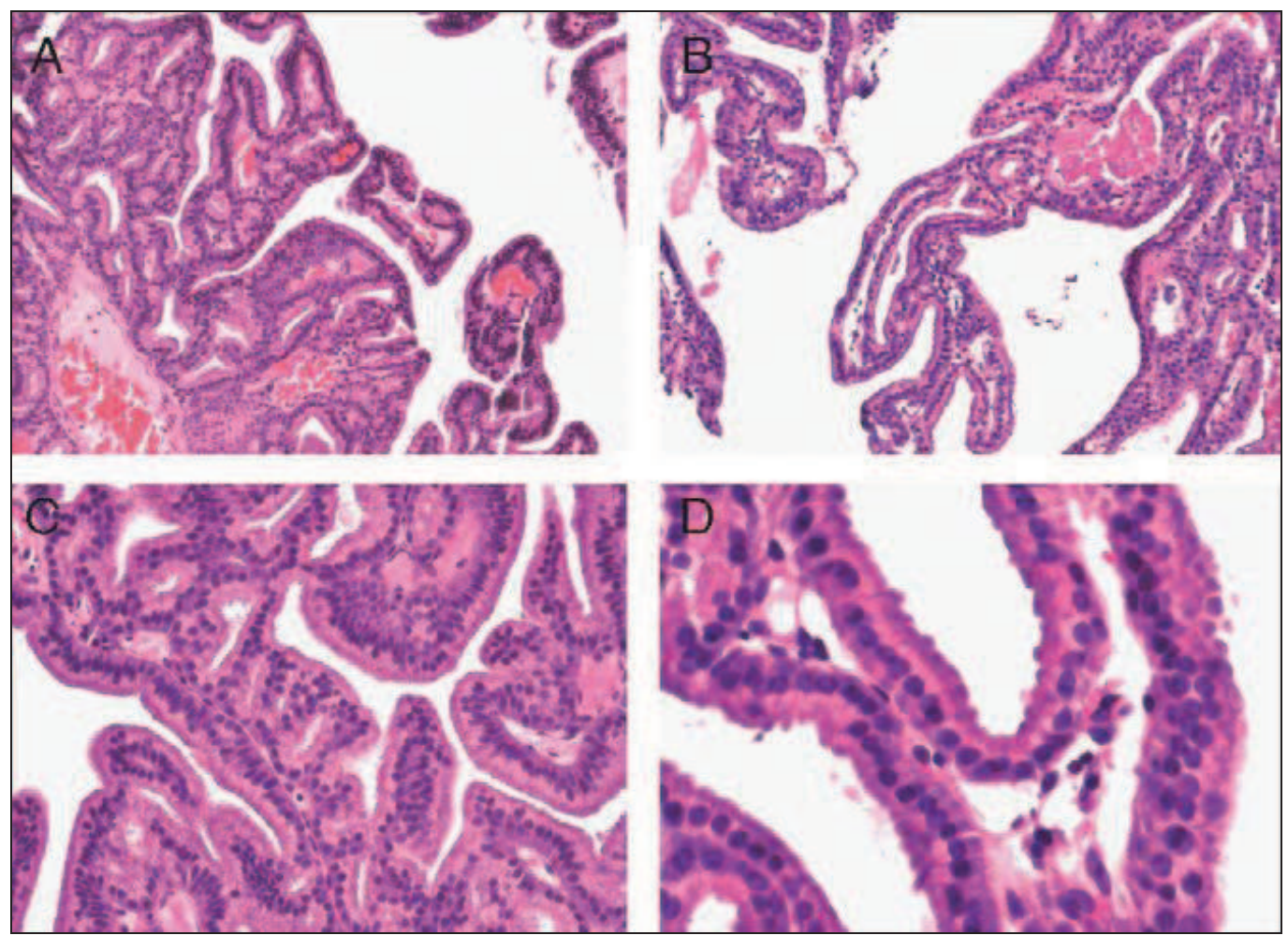

Figure 7 : Adénocarcinome nasosinusien «non intestinal de bas grade » (A-D).

Les papilles sont bordées par une simple assise de cellules cubiques dotées d'un noyau rond, régulier, en position basale et d'un cytoplasme éosinophile. (Jo V et al.[99]).

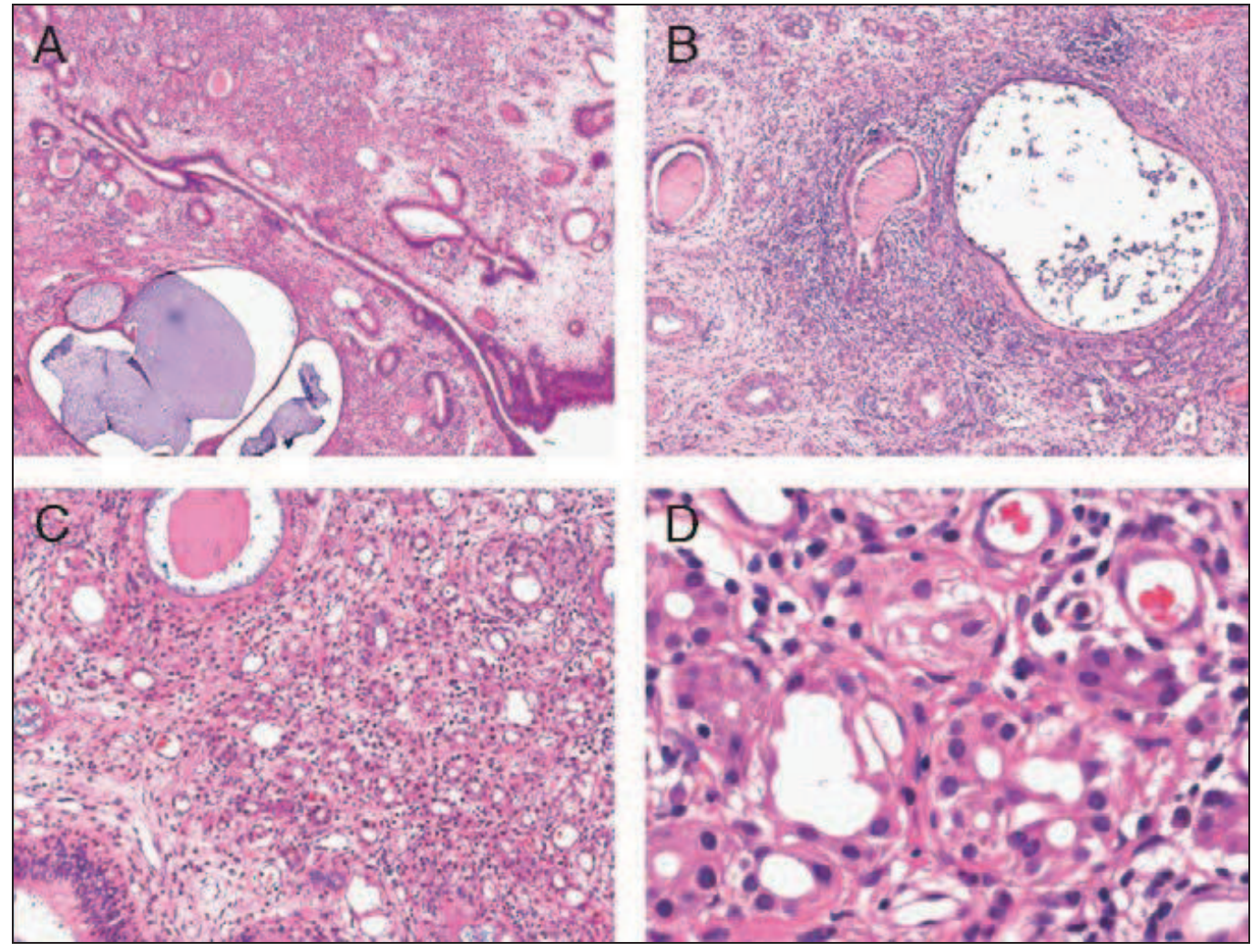

Figure 8: Adénocarcinome nasosinusien « non intestinal de bas grade » (A-D).

Rarement, les structures glandulaires sont le siège de dilatations kystiques. (Jo V et al.[99]). 


\section{- L’adénocarcinome de haut grade :}

II s'agit de tumeurs plus rares, peu différenciées, d'architecture massive, présentant des remaniements nécrotiques, des atypies marquées et de nombreuses figures mitotiques.

Stelow et al. [100] ont étudié 27 cas d'adénocarcinomes nasosinusiens de type non intestinal de haut grade et décrivent plusieurs aspects histologiques : blastomateux, apocrine, oncocytique et peu ou pas différenciés (figures 9 à 13).
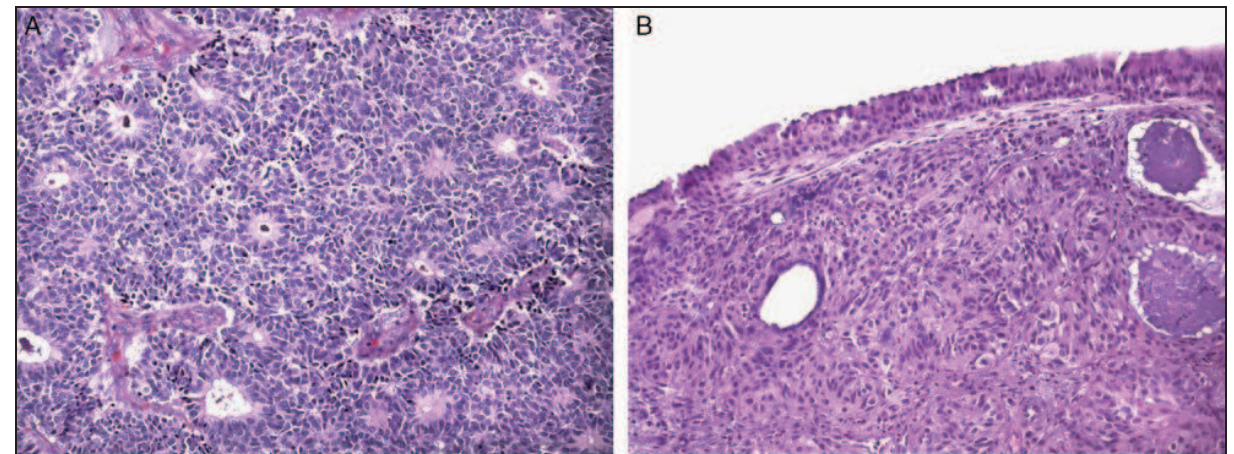

c
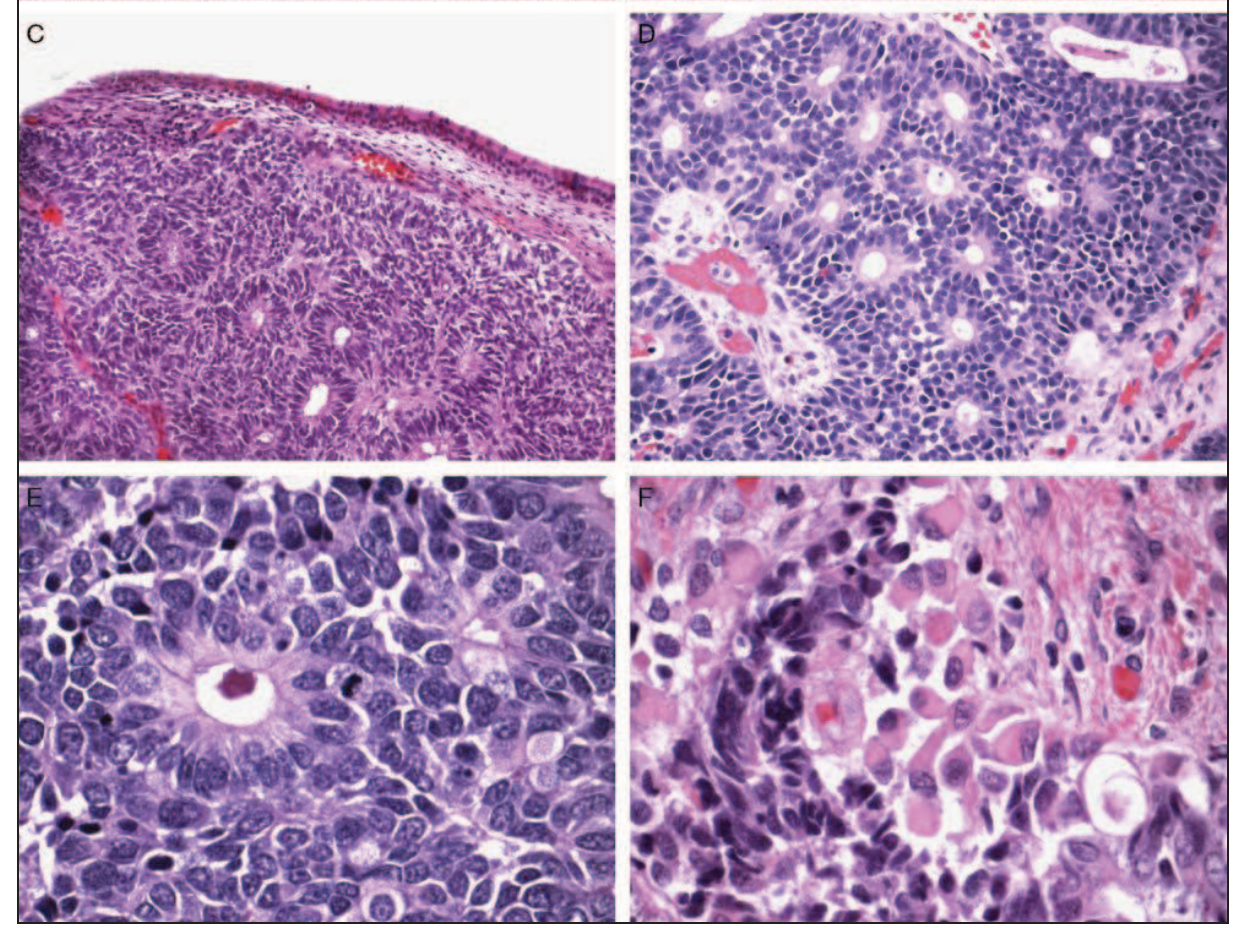

Figure 9 : Adénocarcinome nasosinusien « non intestinal de haut grade », d'aspect blastomateux. (Stelow et al. [100]). 

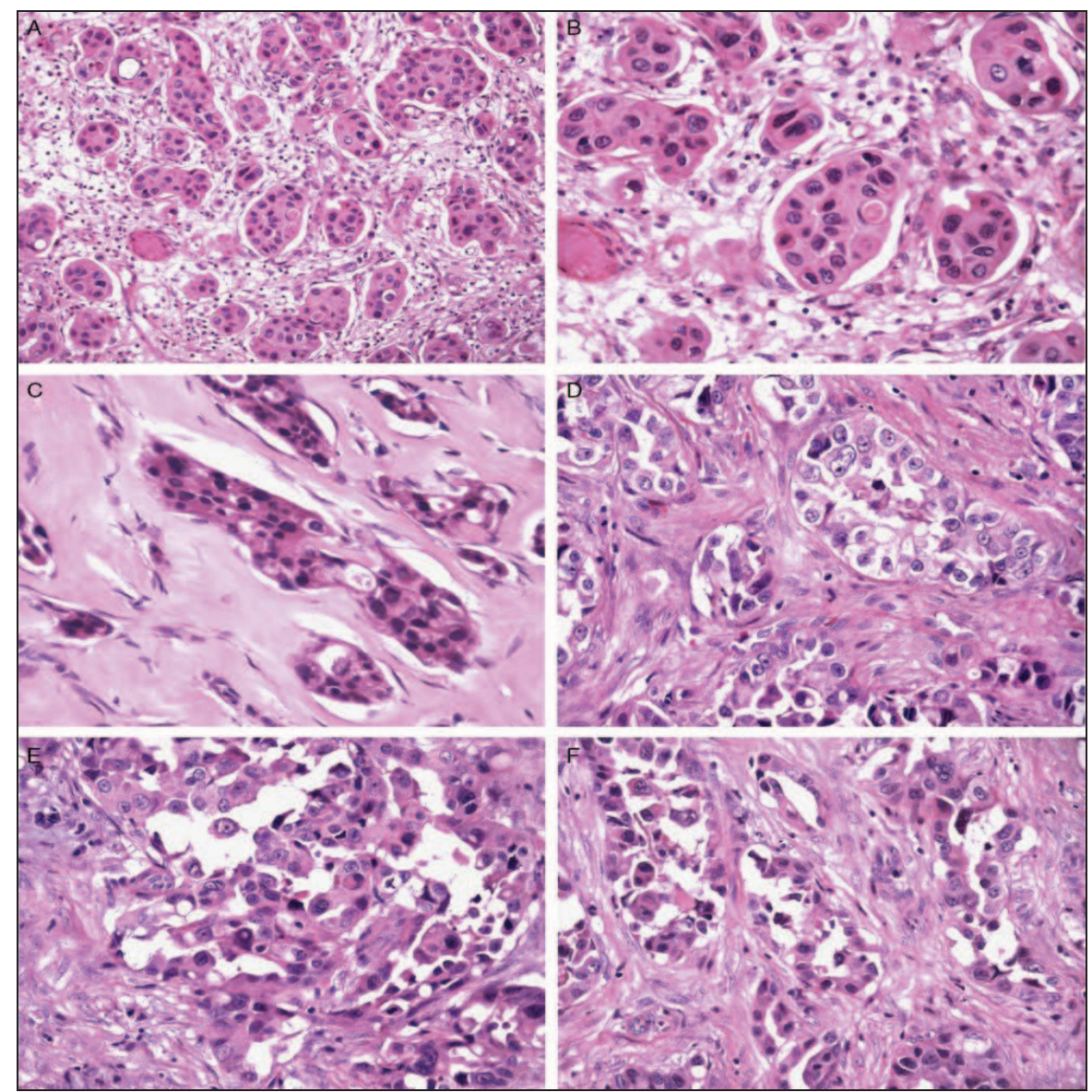

Figure 10: (A-F)

Adénocarcinome

nasosinusien « non intestinal de haut grade », présentant une différenciation apocrine.

(Stelow et al. [100])
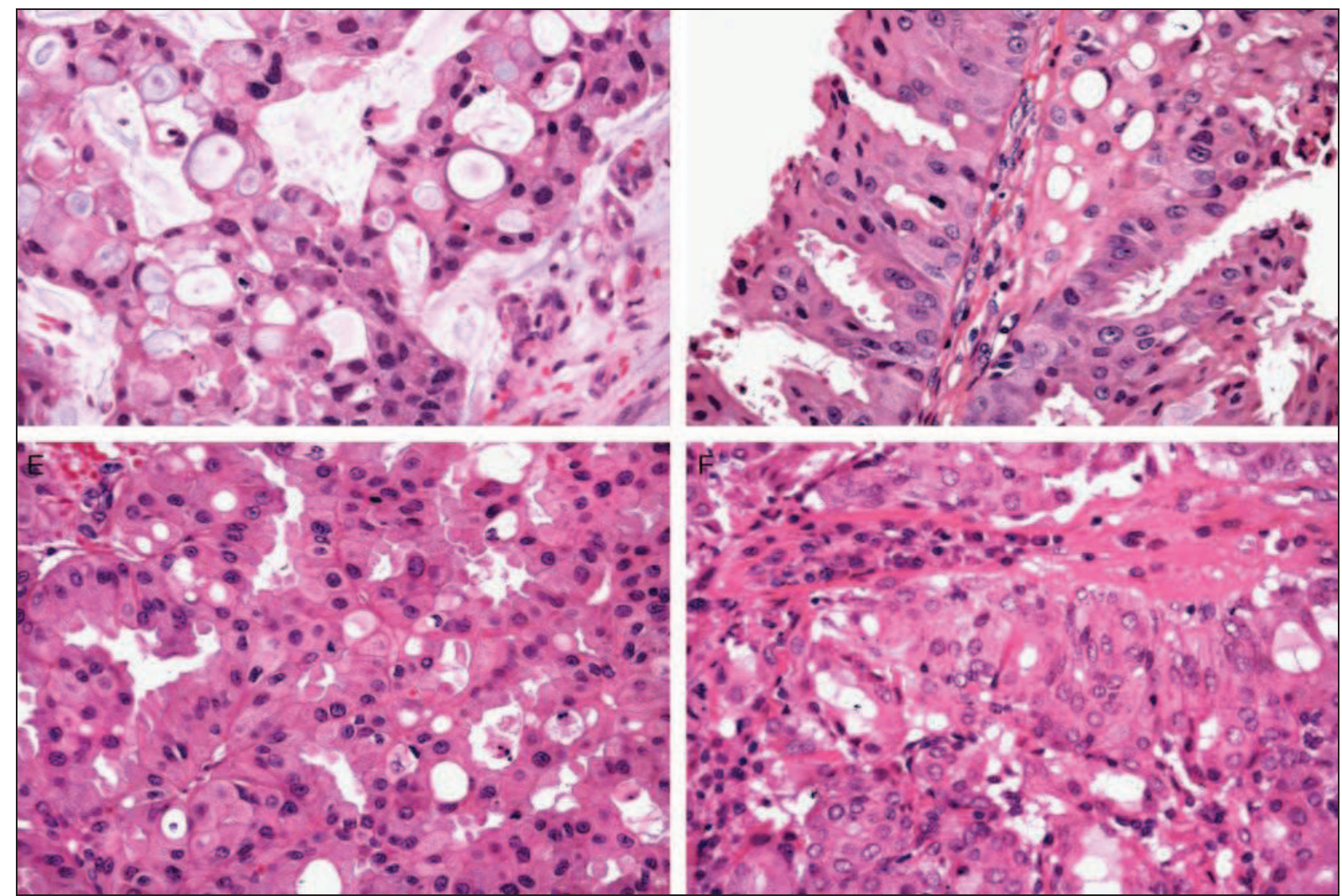

Figure 11: Adénocarcinome nasosinusien « non intestinal de haut grade », oncocytaire. 


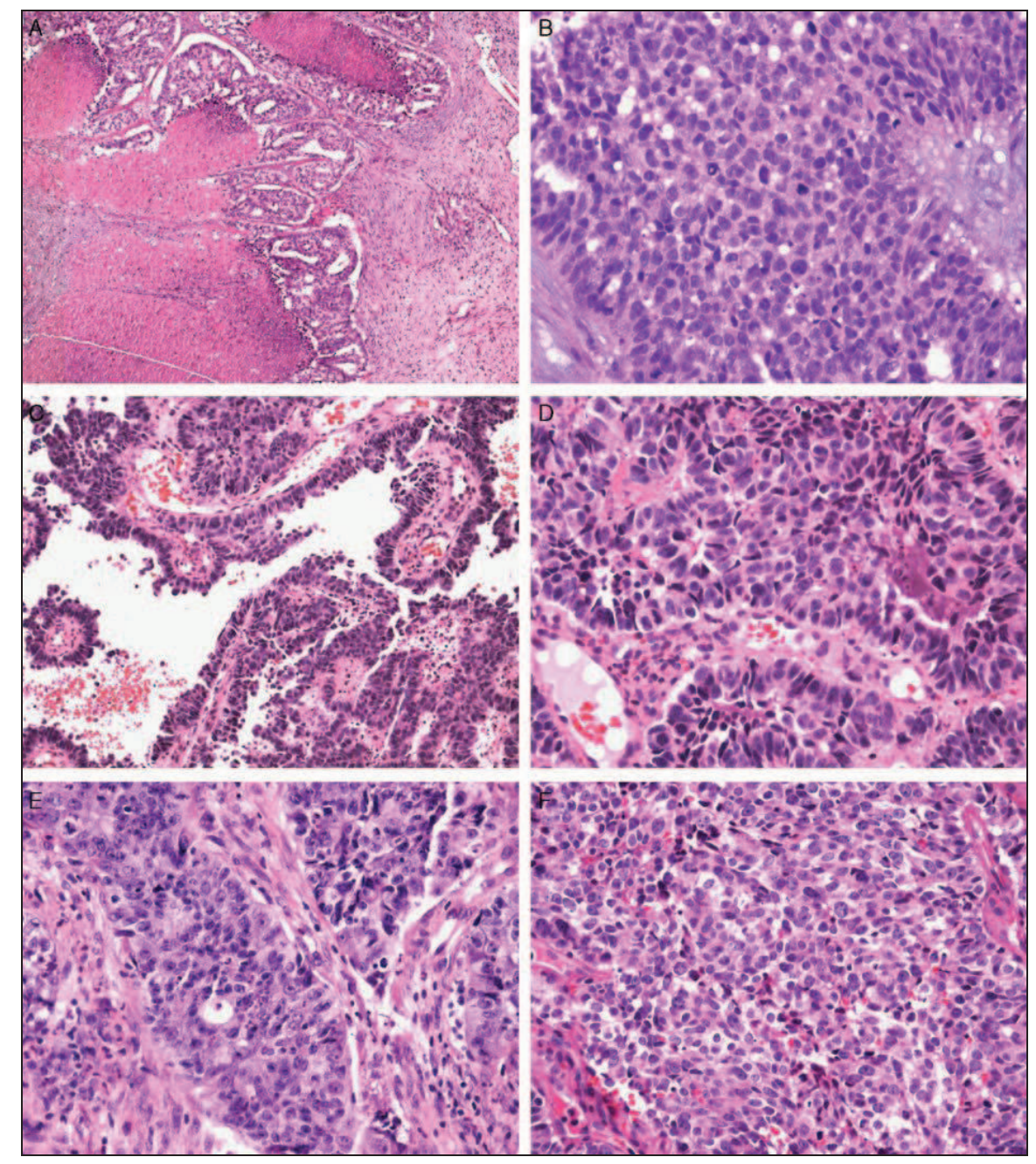

Figure 12: Adénocarcinome nasosinusien «non intestinal de haut grade », peu ou pas différenciés (Stelow et al. [100]).

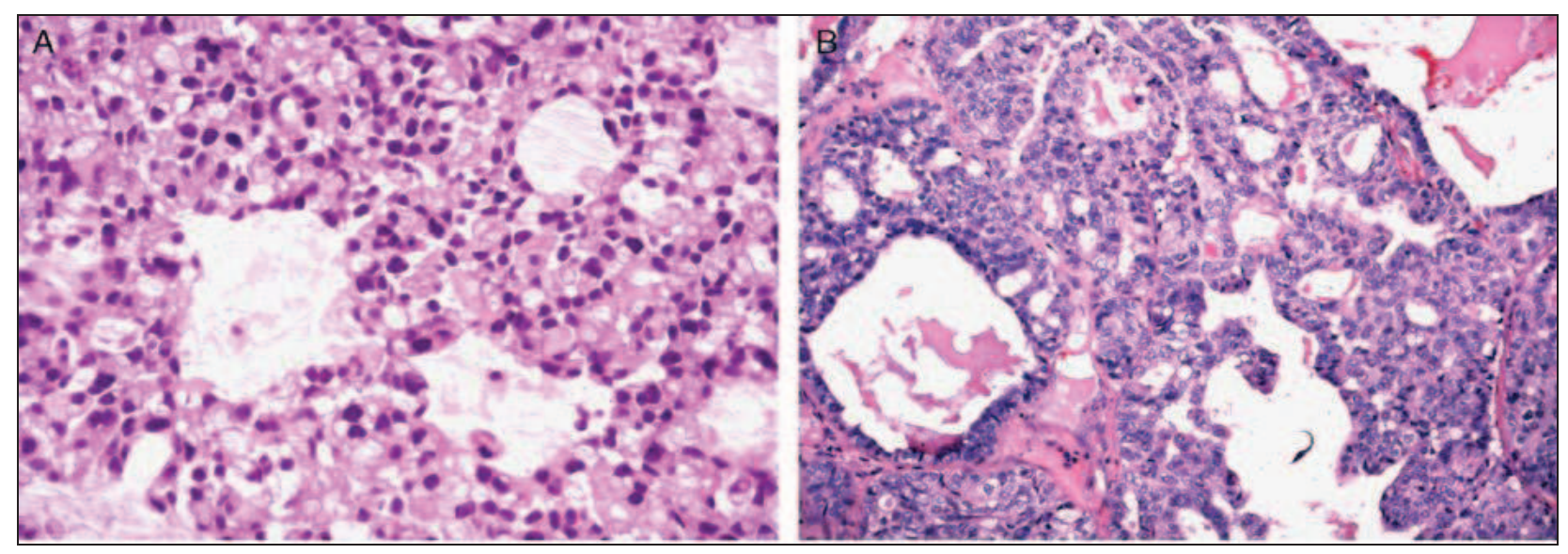

Figure 13: Adénocarcinome nasosinusien « non intestinal de haut grade ».

Rarement, il est observé des territoires à cellules claires $(A)$ ou micro kystiques (B).

(Stelow et al. [100]) 
C - Profil Immunohistochimique

Les adénocarcinomes de type «non intestinal » expriment diffusément la cytokératine 7 (CK 7+) sans expression de la CK 20, du CDX2 ou de la villine.

La négativité des marqueurs myoépithéliaux (actine muscle lisse, calponine) est un argument contre une origine myoépithéliale de ces tumeurs [63][66][67][69].

D - $\underline{\text { Pronostic }}$

a - Adénocarcinomes nasosinusiens de bas grade

Il s'agit de tumeurs beaucoup moins agressives que les ITAC.

Elles peuvent récidiver dans environ $30 \%$ des cas [93 -101].

Les séries les plus récentes d'adénocarcinomes nasosinusiens de type «non intestinal », de bas grade, ne retrouvent pas d'évolution létale.

Tableau 3 : Evolution des adénocarcinomes nasosinusiens de bas grade.

\begin{tabular}{|c|c|c|c|}
\hline Auteurs & Récidives & Métastases & $\begin{array}{c}\text { Décédés de la } \\
\text { maladie }\end{array}$ \\
\hline Heffner et al. [94] & $7 / 23$ & $1 / 23$ & $2 / 23$ \\
\hline Kleinsasser [95] & $1 / 7$ & $1 / 7$ & $1 / 7$ \\
\hline Neto et al. [96] & $3 / 12$ & $0 / 12$ & $0 / 12$ \\
\hline Cathro et Mills [67] & $0 / 7$ & $0 / 7$ & $0 / 7$ \\
\hline Skalova et al. [97] & $2 / 6$ & $0 / 6$ & $0 / 6$ \\
\hline Orvidas et al. [93] & $2 / 5$ & $0 / 5$ & $0 / 5$ \\
\hline Franchi et al. [101] & $1 / 2$ & $0 / 2$ & $0 / 2$ \\
\hline
\end{tabular}


b - Adénocarcinomes nasosinusiens de haut grade

Compte tenu de leur caractère rare, très peu d'études sont rapportées dans la littérature.

Selon l'OMS 2005 [22], ces adénocarcinomes auraient un pronostic plus réservé, avec un taux de survie à 3 ans estimé à $20 \%$.

Heffner et al. [94] recense 14 cas d'adénocarcinomes de haut grade. Douze sont décédés dans les suites de leur pathologie.

Orvidas et al. [93] en rapporte 9. Trois cas ont récidivé et quatre sont décédés.

3 - Diagnostics différentiels des lésions glandulaires bien différenciées des cavités nasosinusiennes

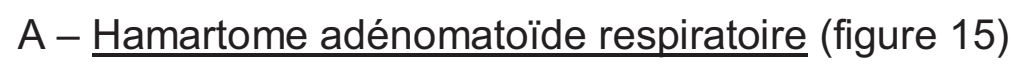

De description récente, il correspond à une lésion polypoïde développée essentiellement au niveau de la portion postérieure du septum nasal, découverte le plus souvent chez le sujet de sexe masculin de 58 ans en moyenne [102-103]

L'axe du polype renferme des glandes de taille petite à moyenne, ramifiées, montrant une architecture lobulaire, sans adossement. Parfois, les glandes semblent s'invaginer à partir du revêtement de surface. 
Elles sont limitées par un épithélium de type respiratoire le plus souvent uni stratifié, par places en métaplasie mucineuse, reposant sur une membrane basale épaissie hyalinisée.

II existe fréquemment dans le stroma au contact, volontiers inflammatoire, quelques glandes séromuqueuses, en continuité avec les glandes à revêtement respiratoire [104-105].

Lorsque les glandes séromuqueuses sont très nombreuses, on parle d'hamartome « séromucineux » (figure 14) [106].

L'épithélium glandulaire montre un phénotype respiratoire : CK7+/CK20-.

II s'agit d'une lésion bénigne qui ne récidive pas après exérèse.

Une étude récente a montré dans 6/18 cas d'adénocarcinomes de bas grade, la présence d'un contingent de type hamartome épithélial adénomatoïde [99]. Pour ces auteurs, ces lésions correspondent plus à des adénomes qu'à des hamartomes et seraient de possibles précurseurs des adénocarcinomes nasosinusiens «non intestinaux » de bas grade.

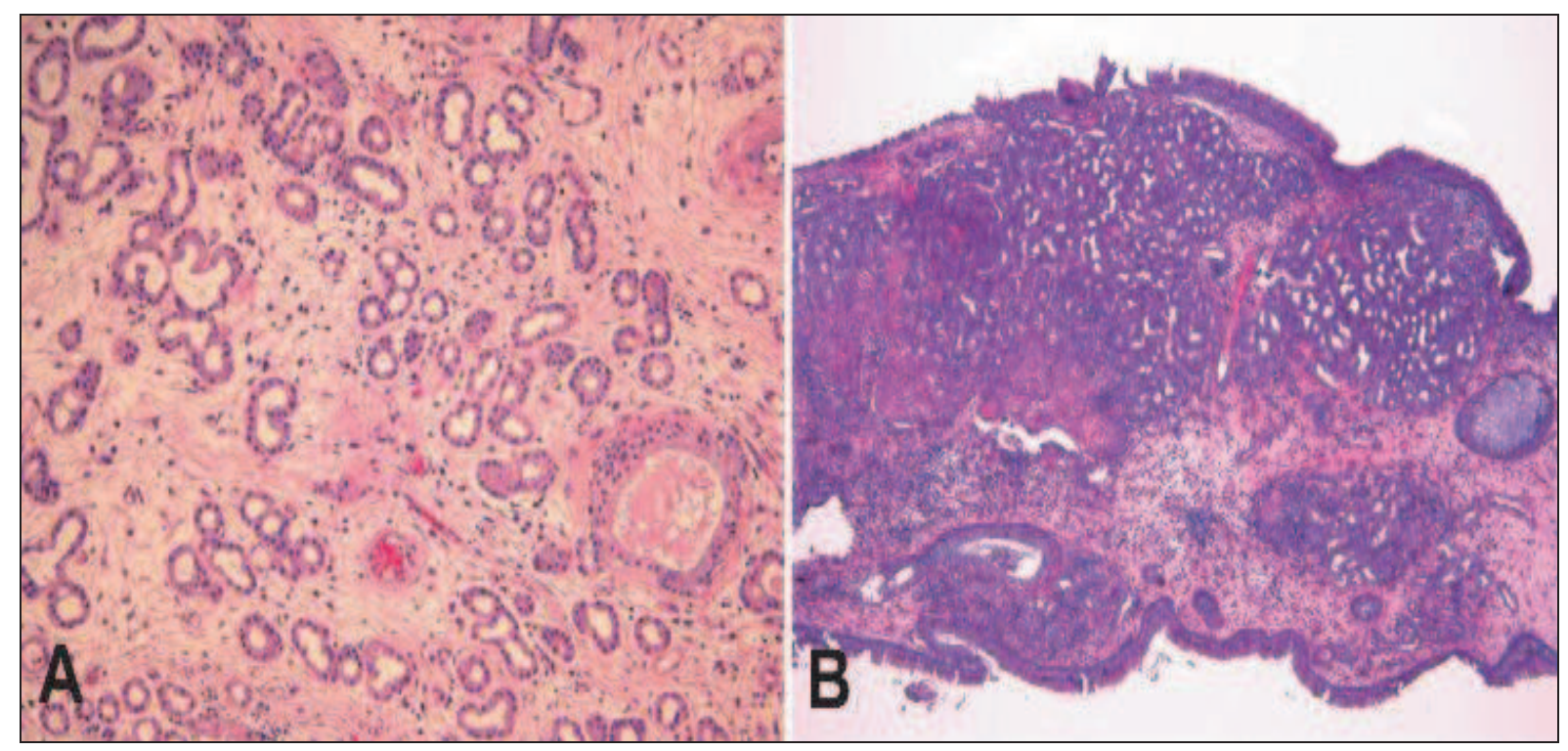

Figure 14: Hamartome séromucineux.

(A) : Nombreuses formations glandulaires de petite taille, disposées au sein d'un chorion œdémateux.

(B) : Architecture globale lobulaire, sans invasion des structures de voisinage.

(Perez-Ordonez [104]). 


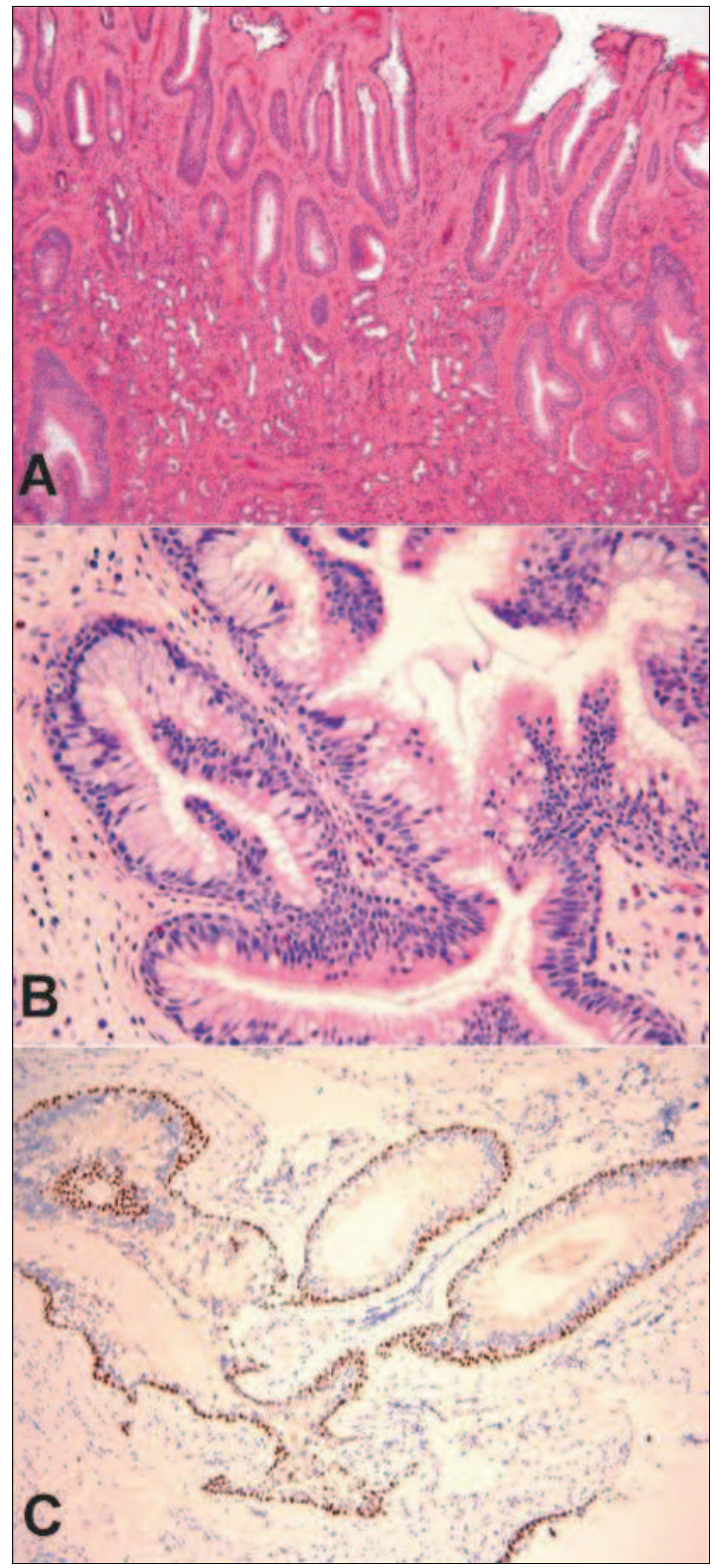

Figure 15:

Hamartome adénomatoïde

respiratoire

(A) : Lésion polypoïde constituée de petites glandes tapissées par un épithélium respiratoire, en continuité avec le revêtement de surface.

(B) : Territoires de métaplasie mucineuse.

(C) : Positivité des cellules basales pour la p63.

(Perez-Ordonez [104]). 


\section{B - Papillome Schneidérien de type inversé (figure 16)}

Cette lésion peut comporter, surtout en surface et sur les papilles exophytiques, un épithélium cylindrique cilié. La composante inversée montre un épithélium toujours épaissi nettement hyperplasique (6 à 30 assises cellulaires) de type transitionnel ou plus rarement malpighien.

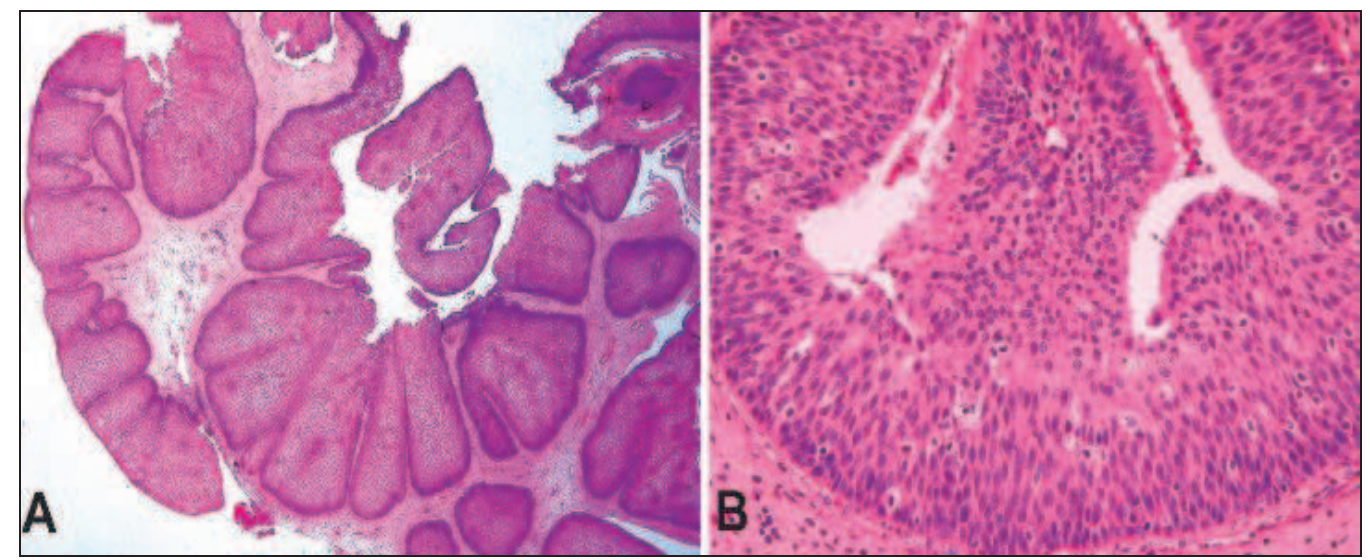

Figure 16 : Papillome Schneidérien inversé

(A) Massifs limités par un épithélium hyperplasique, de type transitionnel, sans axe fibrovasculaire

(B) Microkystes et exocytose d'éléments inflammatoires au sein de l'épithélium. (Perez-Ordonez [104]).

Dans la variante oncocytaire (figure 17), beaucoup plus rare, le revêtement est pluristratifié, fortement éosinophile.

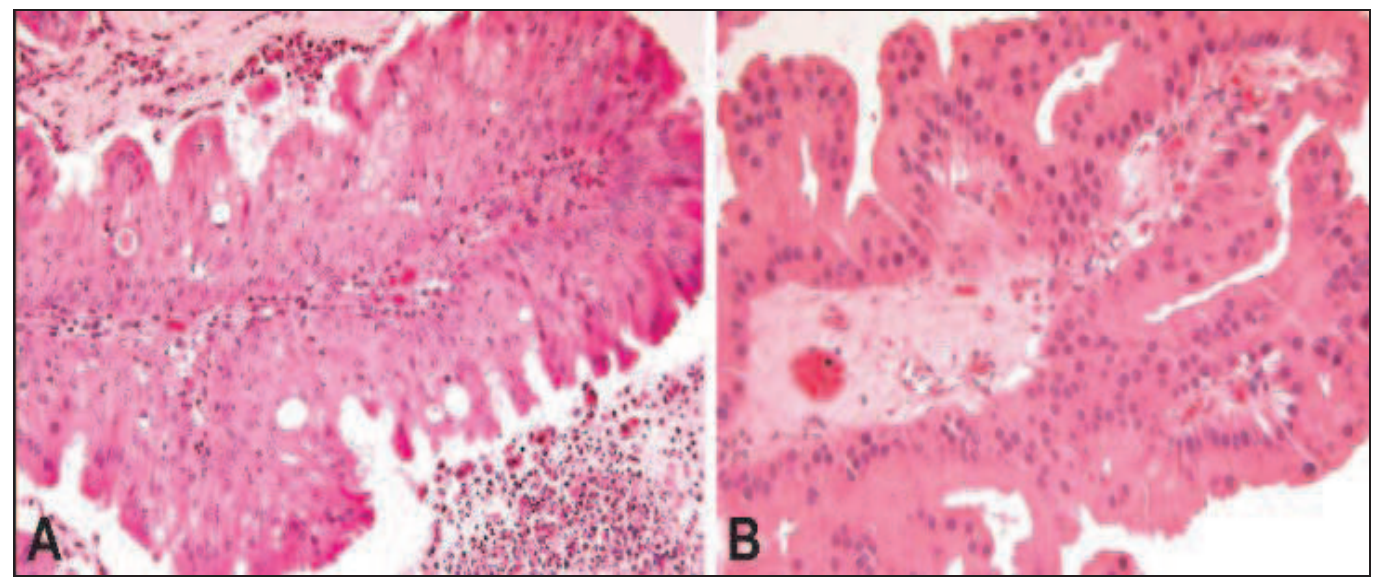

Figure 17: Papillome oncocytique au revêtement pluristratifié, aux cellules éosinophiles, comportant des noyaux ronds réguliers. (Perez-Ordonez [104]). 
Dans ces deux types de papillome Schneidériens, des microkystes intraépithéliaux sont fréquents ainsi qu'une exocytose transépithéliale d'éléments inflammatoires [51].

Les papillomavirus sont surtout impliqués dans les formes exophytiques de papillomes (types 6 et 11).

Néanmoins, le Human Papilloma Virus (HPV) 16 semble présent dans un certain nombre de papillomes inversés et pourrait jouer un rôle dans la transformation de ces lésions [107].

C - Tumeurs développées au dépens des glandes salivaires annexées à la $\underline{\text { muqueuse nasosinusenne }}$

Les plus fréquentes sont l'adénome pléomorphe et le carcinome adénoïde kystique.

La mise en évidence d'un contingent myoépithélial, exprimant l'actine muscle lisse , est un argument pour une tumeur à point de départ salivaire.

Le carcinome à cellules acineuses peut parfois poser des problèmes de diagnostic différentiel avec un adénocarcinome nasosinusien de bas grade : la présence de cellules aux cytoplasmes tantôt clairs, tantôt basophile granuleux, PAS positifs, est un argument pour le carcinome à cellules acineuses [108].

Le carcinome mucoépidermoïde se reconnaît à la présence de différents types cellulaires limitant les cavités glandulaires: cellules claires, malpighiennes et intermédiaires. 


\section{D - Métastase d'un adénocarcinome colorectal}

Les métastases nasosinusiennes sont exceptionnelles: de très rares cas, principalement d'origine rénale (adénocarcinome à cellules claires) et mammaire (adénocarcinome canalaire invasif) ont été rapportés. Elles siègent essentiellement au niveau du sinus maxillaire [109].

Bien que rares, quelques cas de métastases nasosinusiennes d'adénocarcinomes colorectaux ont été décrits. De ce fait, en dehors d'un contexte d'exposition au bois, la découverte d'un ITAC devrait inciter à réaliser, à titre systématique, une exploration endoscopique colorectale.

\section{Tableau 4 :}

Diagnostics différentiels des lésions glandulaires bien différenciées des cavités nasosinusiennes.

\begin{tabular}{|c|c|c|c|c|c|}
\hline Diagnostic & Contexte & Topographie & $\begin{array}{l}\text { Revêtement } \\
\text { glandulaire }\end{array}$ & Particularités & Evolution \\
\hline ITAC & $\begin{array}{c}\mathrm{H}>\mathrm{F}, 60 \text { ans, } \\
\text { exposition } \\
\text { aux } \\
\text { poussières } \\
\text { de bois }\end{array}$ & Ethmoïde (80\%) & $\begin{array}{c}\text { Epithélium } \\
\text { cylindrique mono-ou } \\
\text { pseudo stratifié, avec } \\
\text { cellules } \\
\text { mucosécrétantes }\end{array}$ & $\begin{array}{c}\text { Atypies, mitoses } \\
\text { CK20+, CDX2+, } \\
\text { CK } 7+/-\end{array}$ & $\begin{array}{l}\text { Extensif, } \\
\text { récidive } \\
\text { fréquente, } \\
\text { métastases } \\
\text { rares }\end{array}$ \\
\hline $\begin{array}{l}\text { ADK non } \\
\text { intestinal de } \\
\text { bas grade }\end{array}$ & $\begin{array}{l}\mathrm{H}=\mathrm{F} \\
53 \text { ans }\end{array}$ & $\begin{array}{c}\text { Ethmoïde }(30 \%) \\
\text { Fosse nasale } \\
(20 \%)\end{array}$ & $\begin{array}{l}\text { Epithélium cubique } \\
\text { monostratifié, noyau } \\
\text { régulier en position } \\
\text { basale }\end{array}$ & $\begin{array}{c}\text { Glandes adossées, } \\
\text { parfois kystiques } \\
\text { CK20-, CK7+ }\end{array}$ & $\begin{array}{l}\text { Extensif, } \\
\text { récidive } \\
\text { fréquente, } \\
\text { métastases } \\
\text { rares }\end{array}$ \\
\hline $\begin{array}{l}\text { Hamartome } \\
\text { respiratoire } \\
\text { adénomatoïde }\end{array}$ & $\begin{array}{l}\mathrm{H}>\mathrm{F}, \\
60 \text { ans }\end{array}$ & $\begin{array}{c}\text { Polype de la } \\
\text { partie postérieure } \\
\text { du septum nasal }\end{array}$ & $\begin{array}{l}\text { Epithélium de type } \\
\text { respiratoire, uni ou } \\
\text { pseudo stratifié }\end{array}$ & $\begin{array}{c}\text { Membrane basale } \\
\text { épaissie, glandes en } \\
\text { continuité avec des } \\
\text { acini séromuqueux, } \\
\text { stroma inflammatoire } \\
\text { CK20- , CK7+ }\end{array}$ & $\begin{array}{l}\text { Pas de récidive } \\
\text { après exérèse } \\
\text { simple }\end{array}$ \\
\hline $\begin{array}{c}\text { Papillome } \\
\text { Schneidérien } \\
\text { de type inversé } \\
\text { ou oncocytaire }\end{array}$ & $\begin{array}{l}3 \mathrm{H} / 1 \mathrm{~F} \\
\mathrm{HPV}\end{array}$ & $\begin{array}{l}\text { Paroi latérale des } \\
\text { fosses nasales }\end{array}$ & $\begin{array}{c}\text { Epithélium } \\
\text { acanthosique, de } \\
\text { type respiratoire en } \\
\text { surface, de type } \\
\text { transitionnel (ou } \\
\text { pluristratifié } \\
\text { oncocytaire) dans les } \\
\text { lobules invaginés }\end{array}$ & $\begin{array}{c}\text { Exocytose } \\
\text { d'éléments } \\
\text { inflammatoires, } \\
\text { microkystes intra- } \\
\text { épithéliaux. } \\
\text { CK20-, CK7+ }\end{array}$ & $\begin{array}{c}\text { Extensif, } \\
\text { récidive } \\
\text { fréquente (30\%), } \\
\text { nécessitant une } \\
\text { chirurgie } \\
\text { d'exérèse } \\
\text { complète. } \\
\text { Risque de } \\
\text { transformation } \\
\text { en carcinome } \\
\text { transitionnel }\end{array}$ \\
\hline
\end{tabular}




\section{V - $\underline{\text { Traitement }}$}

Pour de nombreuses équipes [9-21], le traitement repose sur l'association chirurgie et radiothérapie postopératoire, semblant améliorer le contrôle local, la survie globale et la survie sans récidive. L'extension ganglionnaire étant rare, un curage n'est associé qu'en présence d'adénopathie suspecte cliniquement ou radiologiquement lors du bilan initial.

Les voies d'abord chirurgicales sont diverses (transf-faciale, vestibulaire ou endonasale) et sont fonctions de l'extension tumorale.

De la qualité de l'exérèse dépend la qualité de la survie, d'où l'importance d'une exérèse chirurgicale avec limites de résection histologiquement saines.

Les complications postopératoires rencontrées dans 10 à $65 \%$ des cas, sont représentées par les méningites, les saignements et les rhinorhées cérébro-spinales .

La radiothérapie n'a d'indication qu'en postopératoire, où elle permet une amélioration du contrôle local. Les doses délivrées sont en générales de 50 à 70 Grays.

Les chaînes ganglionnaires ne sont pas irradiées de façon systématique devant le faible taux de métastase ganglionnaire dans cette pathologie.

Ses principales complications sont les radionécroses cutanées, les rétractions des tissus mous, les ostéoradionécroses du volet frontal et les atteintes oculaires.

La chimiothérapie n'est classiquement réservée qu'aux situations palliatives. 


\section{I - Famille des récepteurs de croissance épidermique}

Les récepteurs de type Epidermal Growth Factor Receptor (EGFR) sont des molécules transmembranaires permettant à partir de signaux extracellulaires, la transmission d'informations, dont la transduction intracellulaire, provoque un ensemble de processus métaboliques comme la croissance cellulaire, la différenciation, la survie, la progression du cycle et l'angiogenèse [110].

L'EGFR (appelé aussi erbB1 ou HER1) appartient à la famille erbB de récepteurs tyrosine kinase qui comprend trois autres membres: erbB2 (HER2/neu), erbB3 (HER3) et erbB4 (HER4).

Le gène codant pour le récepteur de l'EGF est situé au niveau du bras court du chromosome 7. Il est constitué de 28 exons (200 kb) et code une glycoprotéine d'un poids moléculaire de $170 \mathrm{kDa}$.

Comme de nombreux récepteurs transmembranaires, l'EGFR est composé de trois domaines :

- Extracellulaire NH2-terminal situé dans le milieu extérieur qui contient le site de liaison capable de fixer ses ligands spécifiques ;

- Transmembranaire, hydrophobe, qui permet au récepteur de rester ancré dans la membrane plasmique;

- Intracellulaire $\mathrm{COOH}$-terminal, présentant une activité enzymatique tyrosine kinase. Ce domaine comporte un site de liaison de l'ATP, un site catalytique de l'enzyme et une extrémité riche en résidus tyrosines qui vont subir une phosphorylation. 
Plusieurs ligands peuvent se fixer sur ce récepteur : epidermal growth factor (EGF), transforming growth factor alpha (TGF-alpha), l'amphiréguline, la betaceluline, l'épiréguline, NRG2-alpha (neuregulin 2) et l'heparinbinding EGF.

La fixation d'un de ces ligands sur le récepteur provoque sa dimérisation (soit sous forme d'homodimères "EGFR-EGFR », soit sous formes d'hétérodimères avec d'autres récepteurs de la même famille). Cette modification moléculaire provoque l'activation de sa tyrosine kinase et son autophosphorylation, puis l'activation d'un ensemble de signaux moléculaires intracellulaires.

Ces signaux de transduction sont transmis via différentes voies, dites « de signalisation », qui aboutissent à des effets favorisant ou limitant la prolifération cellulaire et/ou l'apoptose (mort cellulaire programmée) selon un équilibre subtil.

Dans le cas d'une cellule cancéreuse, cet équilibre est rompu, favorisant les effets prolifératifs et antiapoptotiques.

L'EGFR est exprimé dans toutes les cellules de l'organisme, ainsi que dans de nombreuses tumeurs, notamment d'origine épithéliale (cancers colorectaux, pulmonaires, pancréatiques, mammaires ...).

Les principales voies de signalisation activées par l'EGFR sont (figure 18):

- La voie RAS-RAF-MEK-ERK (dite « voie des MAP ou Mitogen-Activated Protein Kinases») qui favorise la prolifération cellulaire.

- La voie PI-3K - AKT, qui favorise la survie cellulaire. 
Figure 18 : Voies de signalisations de I'EGFR (J. Lamoril et al. 2009 [111])

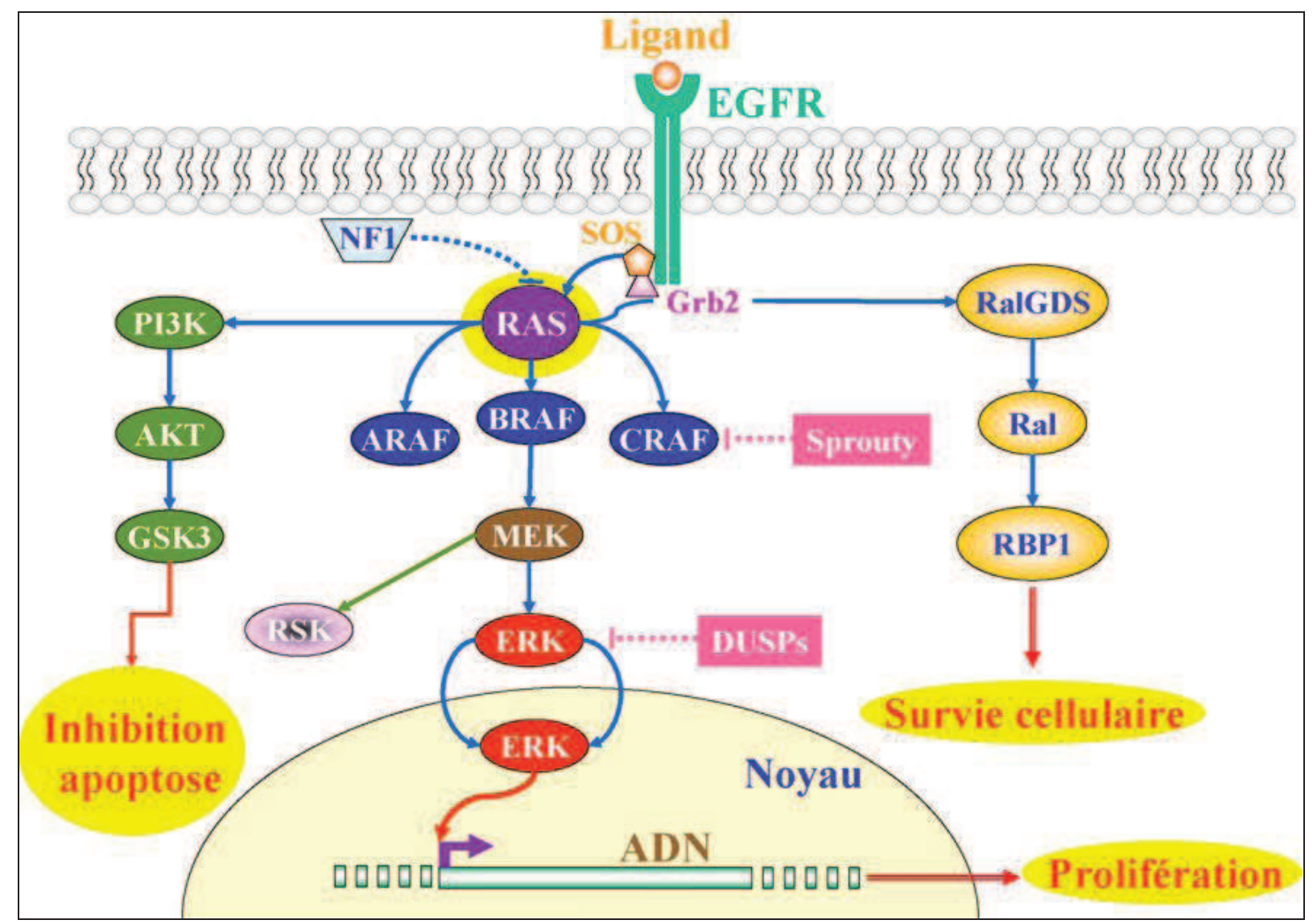

La fixation d'un ligand aboutit à la dimérisation, la phosphorylation du récepteur EGFR et la stimulation de nombreux effecteurs. Les molécules RAS (HRAS et KRAS) sont alors activées par l'intermédiaire de molécules appelées " adaptateurs " telles que growth factor receptor bound protein 2 (Grb2) et son of sevenless (SOS, drosophila, homolog 1 ou guanine nucleotide exchange factor). KRAS activé provoque une cascade moléculaire aboutissant à l'activation de MEK, puis d'ERK et à la transcription de nombreux gènes au niveau du noyau de la cellule (C-MYC, CREB, NF-KB ...) dont l'aboutissement est la prolifération cellulaire, la survie de la cellule, la diffusion de métastases et la néoangiogenèse. D'autres voies sont ég alement activées dont la voie phosphoinositol kinases (PI3K) et AKT ( $v$-akt murine thymoma viral oncogene homolog 1) provoquant l'inhibition de l'apoptose et d'autres signaux moléculaires (par exemple, RSK).

Dans les cancers, l'activation constitutive d'ERK peut être la conséquence de mutations de KRAS, de BRAF ou d'EGFR. L'activation d'ERK est régulée par un rétrocontrôle négatif assuré essentiellement par un ensemble de protéines déphosphorylant ERK et dénommé dual specificity phosphatases (DUSPs).

La protéine Sprouty (appelée aussi drosophila, homolog of, 1; spry1) régule négativement les protéines RAS (et donc KRAS) et indirectement les protéines RAF.

Parmi les protéines sur lesquelles agissent les protéines RAS, deux classes au moins sont activées, des protéines de la famille PI3K (groupe de protéines kinases) et Ras-related guanine nucleotide dissociation stimulator (RalGDS).

PTEN régule la voie de transduction PI3K-AKT. Son inactivation induit l'activation de cette voie.

MEK : mitogen-activated protein kinase extracellular-signal regulated kinase ; ERK : extracellular signal regulated kinase ; Grb2 : growth factor receptor bound 2 ; SOS : son of sevenless; NF1 : neurofibromin 1; Ral : V-ral simian leukemia viral oncogene homolog A ; RBP1: RalA-binding protein 1 ; PTEN : phosphatase homologue to tensin. 


\section{II - Voie RAS - RAF - MEK - ERK}

Cette voie de signalisation intéresse une cascade de kinases dont la dernière a comme cible des facteurs de transcription, dont $C-M Y C$, induisant la prolifération cellulaire et la synthèse d'ADN [110] .

La protéine RAS est localisée sur la face interne de la membrane plasmique à laquelle elle est attachée par un résidu farnésyle.

II s'agit d'une protéine G intermédiaire, située entre les récepteurs et les facteurs de transcription de l'ADN.

Elle a un poids moléculaire de $21 \mathrm{kDa}$. II en existe trois formes différentes : HRAS, KRAS et NRAS.

La cascade de signalisation débute au niveau de la membrane plasmique, par liaison ligand-récepteur, provoquant sa dimérisation. La phosphorylation du récepteur active des GEF ou «facteurs d'échange de guanine », tel que «SOS ». Ces facteurs sont attachés au récepteur par des protéines adaptatrices : « SHC » et « GRB-2 ».

Les protéines GEF poussent RAS à relarguer GDP et à l'échanger contre GTP, ce qui conduit à la forme active de RAS : RAS-GTP, qui agit sur la kinase RAF-1 en provoquant un changement de localisation de cette kinase. La kinase RAF-1 activée induit une phosphorylation en cascade de deux autres MAP-kinases (Mitogen Activated Protein), MEK (MAP-Extracellular signal Regulated Kinase) puis ERK (Extracellular signal Regulated Kinase). Cette dernière, après activation, est transloquée dans le noyau et phosphoryle à son tour différents facteurs de transcription comme FOS, JUN ou MYC. 
Cette voie de signalisation permet de contrôler l'expression de gènes impliqués dans la prolifération cellulaire, la migration cellulaire, l'invasion tissulaire et l'angiogenèse.

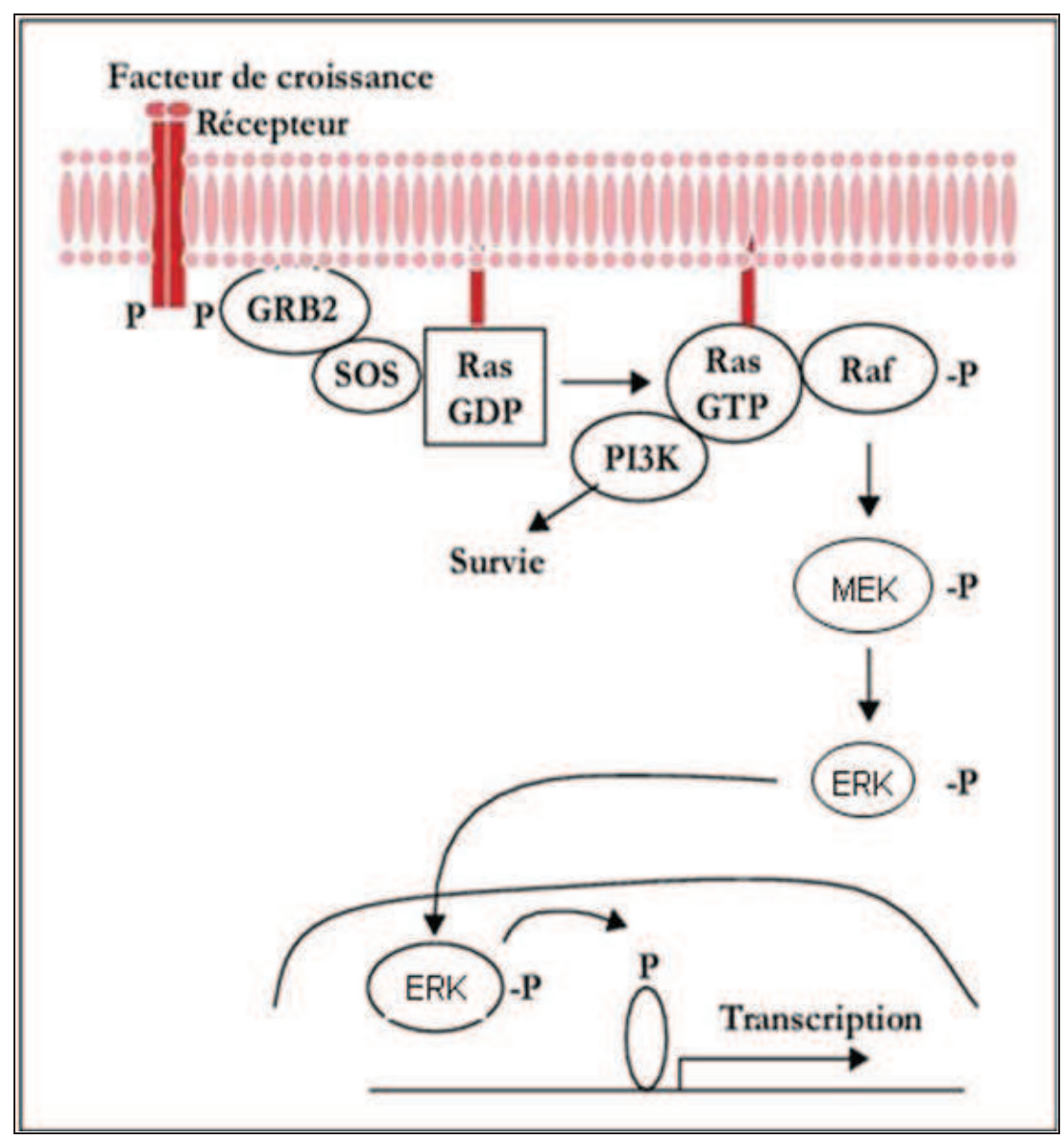

Figure 19: Voie RAS-RAF-MEK-ERK

RAS-GTP active une cascade de kinases par phosphorylation, ce qui a des effets sur la régulation de gènes cibles.

La voie de signalisation RAS/MAPK est anormalement activée dans de nombreux cancers, en particulier le cancer colorectal. Cela est essentiellement lié à l'activation de récepteurs membranaires tels que l'EGFR et les mutations des proto-oncogènes KRAS et BRAF, qui constituent des cibles thérapeutiques potentiellement intéressantes. 


\section{III - Voie PI-3K - AKT - mTOR}

Les membranes lipidiques contiennent des phospholipides, dont certains sont des phosphoinositides.

Le phosphoinositol peut être clivé par les phospholipases $C$ et exercer un rôle de médiateur intracellulaire.

Dans certains cas, les carbones du cycle inositol vont subir une phosphorylation spécifique, par la phophatidylinositol 3 kinase (PI-3K).

Cette molécule sert de point d'ancrage à de nombreuses protéines et permet d'initier des voies de signalisation.

La protéine kinase $B$ (PKB ou $A K T)$ est une sérine/thréonine kinase qui va être activée par PI-3K et qui va phosphoryler de nombreux substrats cytosoliques, dont mTOR.

Cette voie de signalisation aboutit à l'inhibition de l'apoptose et à la prolifération cellulaire [110].

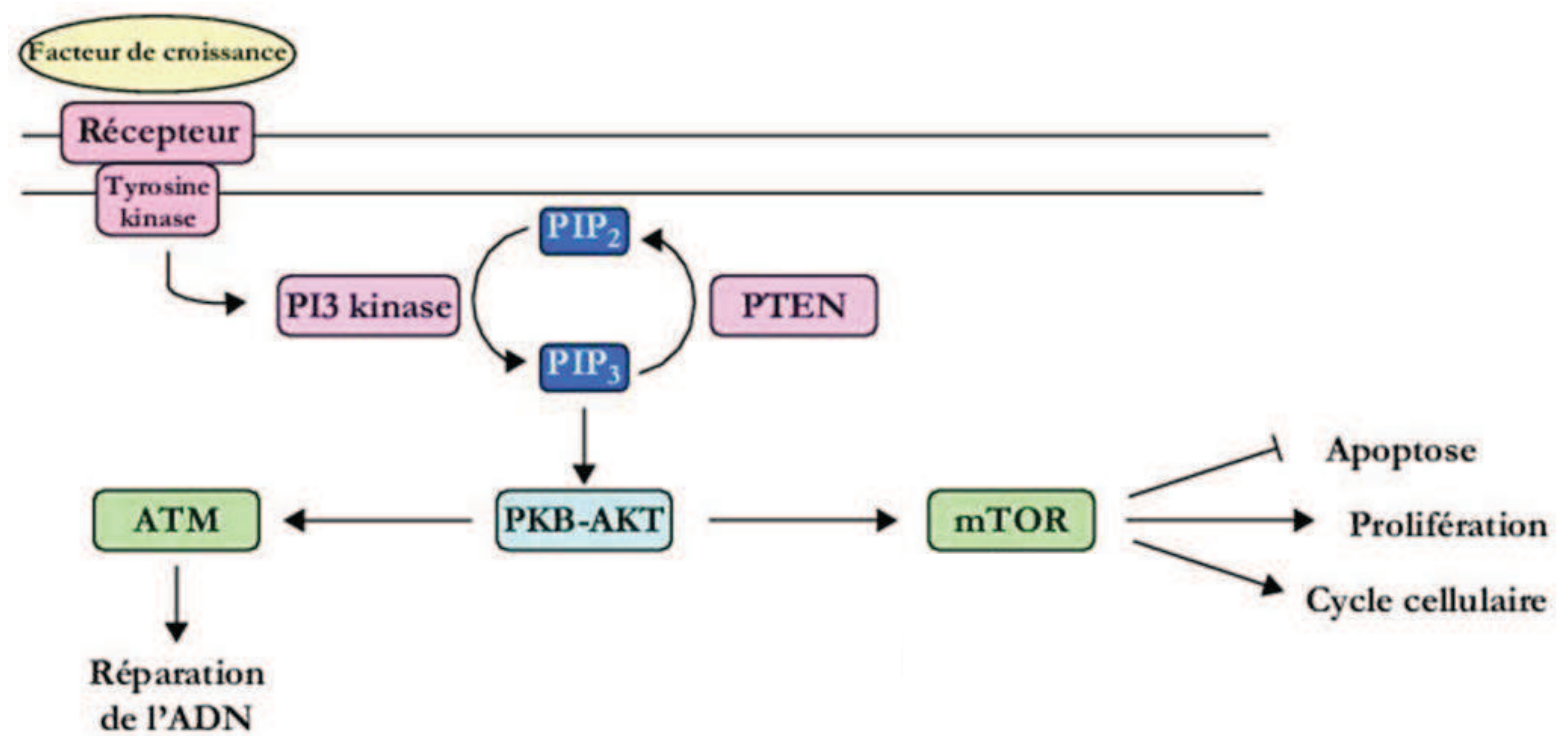

Figure 20: Voie de signalisation PI3K - AKT - mTOR 


\section{IV - Modulation de l'activité de l'EGFR}

La modulation de l'activité de l'EGFR peut schématiquement se faire à différents niveaux et de manière non exclusive [112] :

- Sécrétion par la cellule de son propre ligand, EGF ou TGF- $\alpha$, aboutissant à une activation autocrine du récepteur.

- Surexpression de l'EGFR par régulation transcriptionnelle.

Par exemple, la transcription d'EGFR est modulée par un polymorphisme de séquence de l'intron 1. La variation du nombre de dinucléotides "CA » (entre 14 et 21 " $C A$ ») dans cet intron aboutit à des niveaux d'expression d'ARNm différents.

In vitro, la transcription d'EGFR diminue en cas d'augmentation du nombre de dinucléotides " $\mathrm{CA}$ », alors qu'elle augmente devant une diminution de répétition de ce dinucléotides.

- Modulation de l'activité d'EGFR en fonction du type d'hétérodimère formé et des voies de signalisation activées.

Un homodimère "EGFR-EGFR » induit une prolifération faible et active préférentiellement la voie des MAP kinases, impliquée dans la prolifération et la différenciation cellulaires.

Les hétérodimères comprenant HER2 ou HER3, comme par exemple «EGFRHER2 » et "HER2-HER3 » induisent une prolifération plus élevée. Ces hétérodimères activent préférentiellement la voie PI-3K/AKT également impliquée dans l'inhibition de l'apoptose ;

- Surexpression pathologique de l'EGFR par augmentation du nombre de copies du gène sur le même chromosome (amplification) ou par augmentation du nombre de chromosomes 7 (polysomie);

- Apparition de mutations oncogéniques activatrices. 
Dans plusieurs processus néoplasiques, l'EGFR devient un oncogène par acquisition d'une mutation activatrice. Le récepteur est alors activé de façon constitutive et cela en l'absence de ligand.

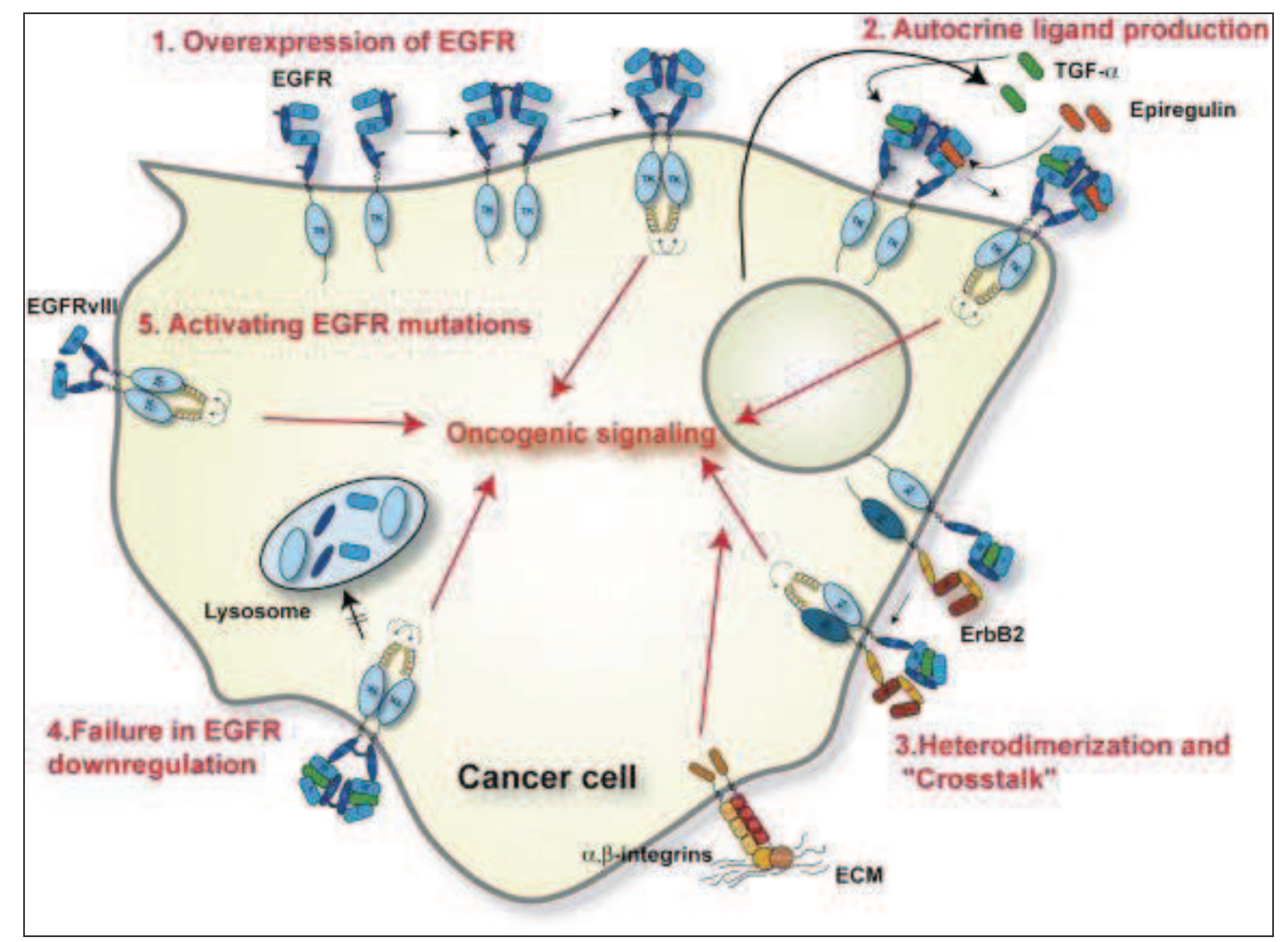

Figure 21:

Voies de signalisation EGFR et carcinogenèse (Zandi et al. [112]) 
Les thérapies ciblées anticancéreuses utilisent des molécules dirigées spécifiquement contre les voies de signalisation activées dans les cellules tumorales, contrairement aux chimiothérapies conventionnelles, dirigées contre les cellules proliférantes, tumorales ou non.

\section{I - Les anticorps monoclonaux et les inhibiteurs de Tyrosine Kinase}

L'ensemble des propriétés d'EGFR et leur dérégulation au cours des processus tumoraux a abouti au développement de molécules ciblant celle-ci [113]. II existe deux stratégies thérapeutiques « anti-EGFR » :

\section{- Les anticorps monoclonaux}

Exemples : Cetuximab (Erbitux*); Panitumumab (Vectibix $\left.{ }^{*}\right)$.

Ces anticorps se lient à l'ectodomaine de l'EGFR, dans sa configuration inactive et inhibent ainsi de façon compétitive la fixation des ligands endogènes au récepteur. Leur efficacité résulte possiblement de 3 mécanismes : l'inhibition de la cascade de transduction dépendante de l'EGFR ; sa " down régulation » et sa dégradation ; et l'activation d'une réponse immunitaire de type cytotoxicité dépendante de l'anticorps ou phénomène « $A D C C$ ».

- Les inhibiteurs du domaine phosphotyrosine kinase intracellulaire (TKI)

Exemples : ZD 1839 Gefitinib (Iressa*) et OSI-774 Erlotinib (Tarceva*).

II s'agit d'inhibiteurs sélectifs du domaine tyrosine kinase du récepteur à l'EGF. Ils bloquent la transduction du signal et la multiplication cellulaire. 


\section{II - Facteurs prédictifs de réponse}

Malgré l'intérêt évident de ces thérapies ciblées, la disparité de la réponse clinique aux " anti-EGFR » souligne la nécessité d'identifier des bio marqueurs prédictifs de sensibilité ou de résistance au traitement, pour une utilisation plus pertinente de ces molécules [110][114].

1 - Statut mutationnel de l'EGFR

- Principales mutations oncogéniques et sensibilité accrue aux « anti-EGFR »

Dans de nombreuses tumeurs, les mutations oncogéniques sont retrouvées au sein des exons 18 à 21 codant le domaine tyrosine kinase du récepteur.

Elles se situent au niveau du site de fixation de l'ATP qui est aussi le site de fixation des « anti-EGFR » de type «TKI».

Dans le cancer broncho-pulmonaire non à petites cellules, plusieurs altérations principales ont été associées à une sensibilité aux «TKI » :

Mutation ponctuelle au sein de l'exon 18 : G719A/C

Mutations ponctuelles au sein de l'exon 21 : L858R et L861Q

Délétion en phase au sein de l'exon 19 des codons 746 à 750 (E746-A750del).

- Mutations oncogéniques rares et résistance aux « anti-EGFR »

Certaines mutations de l'EGFR confèrent une résistance aux « anti-EGFR ». Elles sont rarement retrouvées d'emblée (résistance primaire), mais le plus souvent sélectionnées au cours du traitement par un TKI (résistance secondaire). 
Les résistances primaires sont associées à plusieurs types de mutations ponctuelles ou des insertions rares de l'EGFR, situées au niveau de l'exon 18 ou 20.

Les résistances secondaires apparaissent environ six à neuf mois après observation d'une réponse clinique initiale, sous traitement par TKI. Elles correspondent à la sélection d'un clone tumoral qui a développé un mécanisme de résistance.

Environ $50 \%$ des patients sélectionnent un clone tumoral résistant en rapport avec une mutation T790M, située au niveau de l'exon 20 de l'EGFR. Celle-ci se caractérise par un remplacement d'une thréonine par une méthionine au niveau du codon 790 et aboutit à un changement de conformation de la protéine, empêchant la fixation des TKI.

Une autre mutation de résistance, la mutation D761Y dans l'exon 19, est retrouvée de façon beaucoup plus rare.

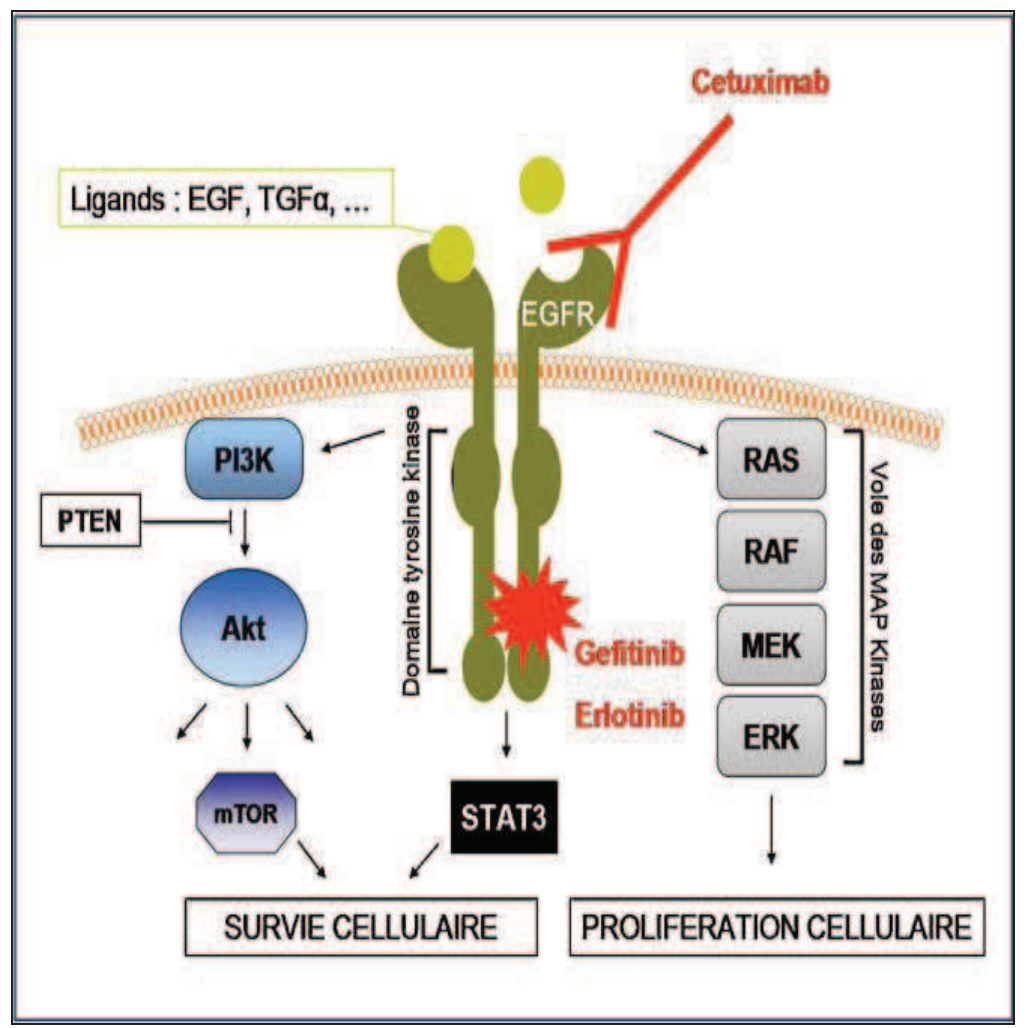

Figure 22 : Représentation schématique d'Epidermal Growth Factor Receptor (EGFR) et des voies de signalisation sous-jacentes, ainsi que du mécanisme d'action des principaux inhibiteurs d'EGFR (A.B. Cortot [113]). 


\section{TIIIIIIX IIII IIIIII}
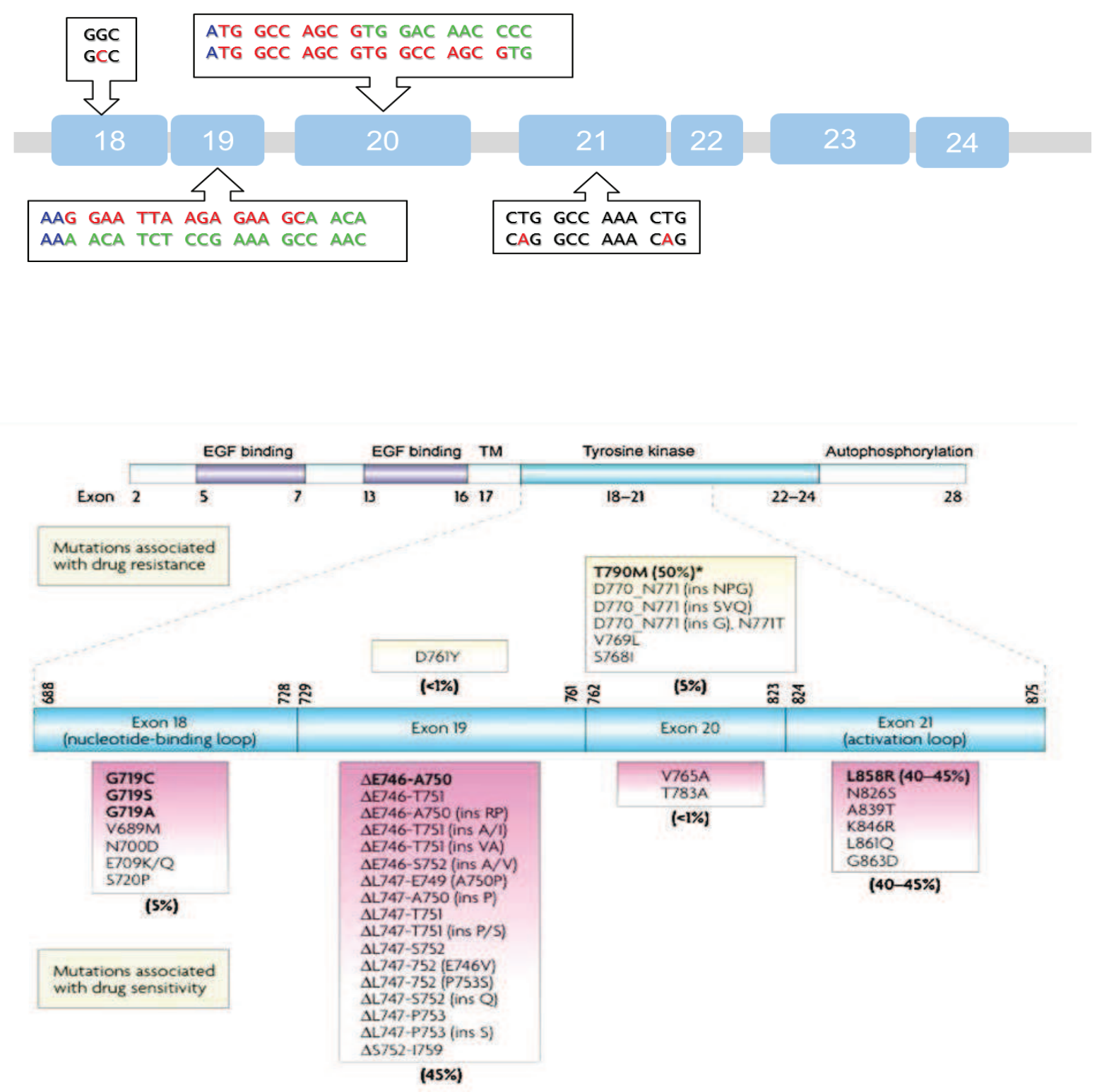

Mutations oncogéniques de l'EGFR:

- Mutations ponctuelles faux sens des exons 18, 20 et 21 (719, 790, 858, 861).

- Insertion/duplication en phase de l'exon 20.

- Délétion en phase de l'exon 19 (4 à 6 acides aminés). 


\section{2 - Statut mutationnel KRAS}

II est maintenant établi que les mutations du gène KRAS, en aval de l'EGFR, ont un rôle prédictif majeur de la résistance au traitement " anti-EGFR » de type anticorps monoclonaux. Ces mutations affectent surtout le codon 12 de l'exon 2 (80\% des cas), moins fréquemment le codon 13 de l'exon 2, et encore plus rarement le codon 61 de l'exon 3, un autre codon, ou les codons 12 et 13 simultanément. Elles sont retrouvées aussi bien dans la tumeur primitive que dans les métastases, avec une excellente corrélation.

Les mutations sur le gène KRAS jouent un rôle fondamental dans l'activation de la protéine KRAS mutée et la progression du processus tumoral.

Les codons 12 et 13 codent pour une glycine située à proximité du site catalytique de la protéine provoquant en cas de mutation, une diminution de l'activité GTP ${ }^{\text {ase }}$ de la molécule qui reste alors dans la conformation active liée au GTP. Par cascade en chaîne, cette activation chronique de la protéine RAS active les autres voies métaboliques (PI-3K et MAP kinases notamment) aboutissant à la transformation maligne. Cette activité étant constitutive, elle ne répondra pas au blocage d'EGFR (situé en amont de KRAS dans la cascade métabolique) par des molécules spécifiques. La cellule sera donc résistante aux « anti-EGFR ».

Selon la mutation causale, cette diminution d'activité est variable et, par conséquent, l'agressivité de la tumeur aussi. A titre d'exemple, la mutation au codon 12 de la glycine en valine (p.G12V) est associée à une plus grande agressivité des tumeurs colorectales.

Ainsi, la recherche de mutations du gène KRAS est maintenant une étape préliminaire indispensable avant toute prescription d'une thérapie ciblée «antiEGFR ». 


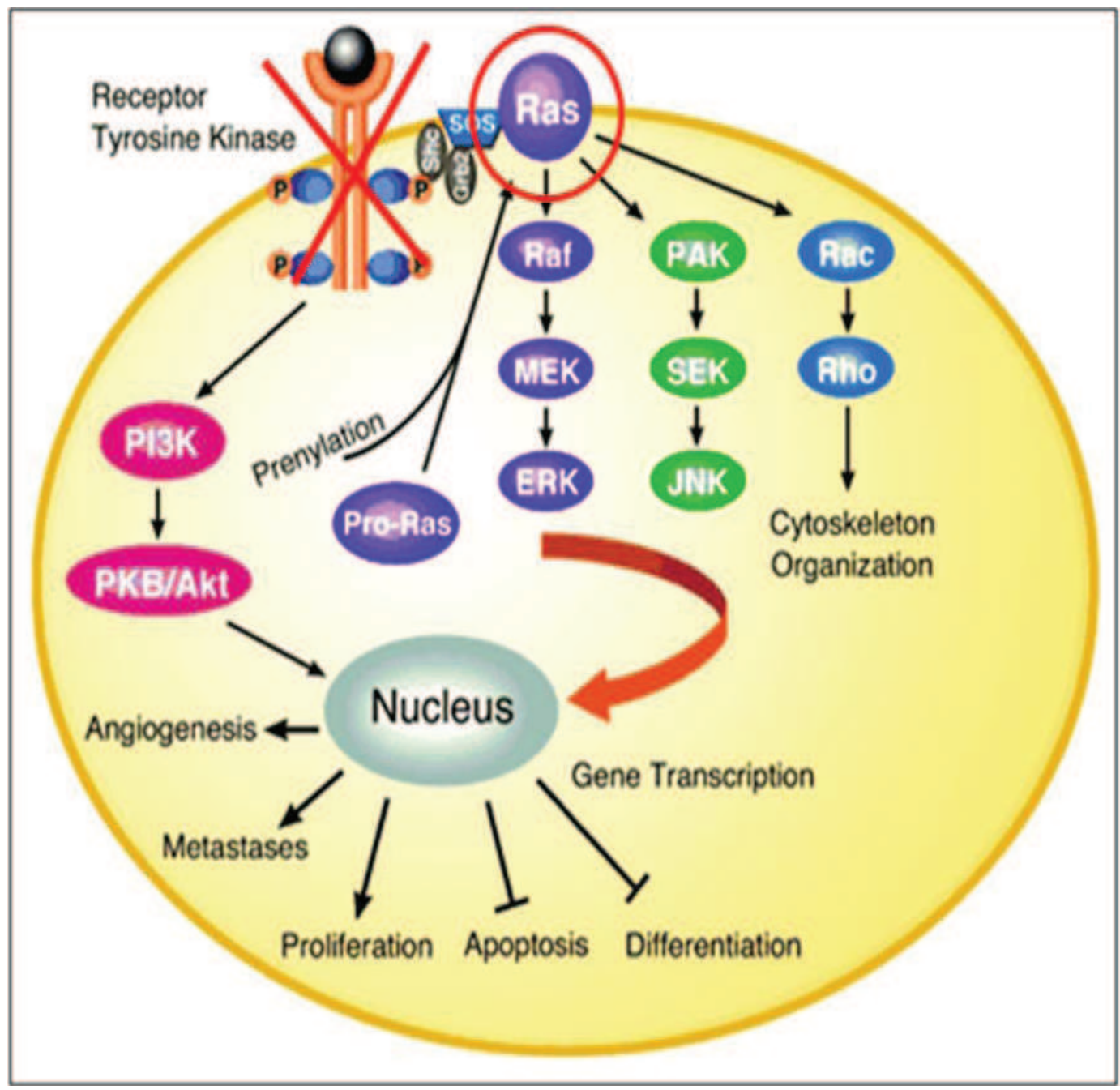

Des mutations somatiques de l'oncogène KRAS sont retrouvées dans de très nombreuses tumeurs et dans 35 à $40 \%$ des cancers colorectaux [115]. Ces mutations somatiques entraînent une modification de la conformation de la protéine mutée qui a pour conséquence une stabilisation de la liaison de la protéine KRAS au GTP. Cette liaison stable au GTP est à l'origine d'activations constitutives découplées de la voie de transduction du signal située en aval. Des signaux de mobilité cellulaire, de croissance cellulaire, d'angiogenèse et anti-apoptotiques sont alors produits par la cellule néoplasique porteuse de cette mutation. Les mutations de KRAS touchent essentiellement les codons 12 et 13 de l'exon 2 (c.34G, c.35G du codon 12 et c.37G, c.38G du codon 13). 
Les trois objectifs de ce travail étaient de :

- Rapporter l'expérience Rouennaise à propos d'une série de 41 patients porteurs d'adénocarcinome nasosinusiens recensés dans le service d'ORL et d'Anatomie et de Cytologie Pathologiques entre 1986 et 2010.

- Caractériser le phénotype de ces tumeurs rares et évaluer l'expression de la protéine EGFR par des techniques immunohistochimiques.

- Analyser le génotype de ces proliférations tumorales en recherchant la présence d'altérations somatiques des gènes EGFR et KRAS, via des techniques de biologie moléculaire, afin d'identifier leurs potentielles sensibilités / résistances aux thérapies ciblées « anti-EGFR ». 


\section{$\underline{\text { I-Données cliniques }}$}

L'étude a porté sur 41 patients atteints d'un adénocarcinome nasosinusien, diagnostiqué dans les services d'ORL et d'Anatomie et de Cytologie Pathologiques du CHU de Rouen, entre le 01/01/1986 et le 31/12/2010.

Les données cliniques ont été recueillies dans le dossier médico-chirurgical de chaque patient.

Les informations répertoriées sont (cf annexe1, fiche du service d'ORL) :

- Le sexe et l'âge au moment du diagnostic initial.

- L'activité professionnelle avec la notion d'une exposition à un facteur de risque, en particulier au bois, la durée de cette exposition ainsi que l'intervalle entre le début de l'exposition et le diagnostic initial.

- La symptomatologie fonctionnelle et sa durée d'évolution.

- Les données des examens cliniques et para-cliniques, précisant le site de la lésion tumorale et son extension.

- Les différents traitement effectués : chirurgie, radiothérapie, chimiothérapie.

- La durée du suivi, la survenue éventuelle de récidives, ainsi que du décès du patient.

- L'estimation de la survie.

La classification clinico-radiologique internationale TNM UICC 2010 a été appliquée aux 41 cas de notre étude. 
Annexe 1 


\section{$\underline{\text { II Données anatomo-pathologiques }}$}

\section{$1-\underline{\text { Macroscopie }}$}

Les données macroscopiques, relevées dans le compte-rendu anatomopathologique concernaient les adénocarcinomes nasosinusiens.

Pour chaque patient, la tumeur primitive a été systématiquement analysée, soit sur biopsie, soit sur pièce d'exérèse chirurgicale, soit les deux (dans les cas où un traitement complémentaire aurait été effectué entre les deux actes, à type de radiothérapie ou chimiothérapie), le choix ayant porté sur la qualité du prélèvement et le pourcentage de cellules tumorales.

Par ailleurs, les récidives locales ou métastatiques ont, en fonction de l'histoire clinique, également été étudiées.

Sur les 41 patients, 3 ont été exclus en raison de l'absence de matériel analysable, soit au total 38 patients inclus dans cette étude.

Cela correspondait à 54 échantillons, dont 39 pièces opératoires et 15 biopsies.

2 - Morphologie en colorations usuelles

L'ensemble des coupes histologiques colorées par l'hématéine-éosine-safran (HES), correspondant aux tumeurs initiales plus ou moins aux récidives et aux métastases a été relu.

D'autres colorations (telles que le PAS ou le Bleu Alcian) ont parfois été utilisées, afin de mieux évaluer le caractère mucosécrétant de certaines tumeurs.

La classification histologique appliquée à ces tumeurs correspond à la classification de Barnes, selon l'OMS 2005. 


\section{3 -Immunohistochimie}

Une étude immunohistochimique complémentaire a été réalisée avec les anticorps CDX2 (anticorps monoclonal de lapin, «EPR2764Y », Cell Marque®), cytokératine 20 (anticorps monoclonal de souris, "Clone Ks 20.8", Dako®), cytokératine 7 (anticorps monoclonal de souris, "Clone OV-TL 12/30", Dako®) et EGFR (anticorps monoclonal de souris, "CONFIRM anti-EGFR 3C6", Ventana $\left.{ }^{\circledR}\right)$, pour l'ensemble des lésions tumorales primitives.

Les immunomarquages ont été effectués par l'automate BenchMark XT (Ventana Medical System®), sur coupes déparaffinées de $5 \mu \mathrm{m}$, de tissus fixés dans le formol.

Le système de révélation utilisé (ultraView Universal DAB Detection Kit de Ventana Medical Systems $\left.{ }^{\circledR}\right)$ mettait en évidence, s'il y avait lieu, l'antigène recherché, réaction traduite par une coloration brune, alors que le reste de la lame était colorée en bleue par l'hémalun (figures $25_{A}$ et $25_{B}$ ).

\section{$A$ - Le facteur de transcription CDX2}

Le gène $C D X 2$ code pour un facteur de transcription exprimé au niveau des noyaux des cellules épithéliales de la muqueuse intestinale, du duodénum au rectum. II joue un rôle clé dans la régulation de la prolifération et de la différenciation cellulaire ainsi que dans l'établissement et le maintien du phénotype intestinal au cours du développement [116].

Son expression a été observée dans les cellules épithéliales intestinales adultes normales telles que les cellules absorbantes, les cellules caliciformes, les cellules endocrines et les cellules de Paneth.

Néanmoins, une expression disséminée a été signalée dans les ductules pancréatiques. Aucune expression n'a été observée dans d'autres tissus/cellules normaux testés. 
L'expression de CDX2 a été rapportée dans 86 à $100 \%$ des adénocarcinomes colorectaux, primitifs et métastatiques, mais aussi au niveau des adénocarcinomes gastriques (22 à 70\%), des carcinoïdes du tractus gastro-intestinal, des adénocarcinomes mucineux de l'ovaire (11-100\%), des adénocarcinomes vésicaux (47-100\%), pancréatiques (32-60\%) et prostatiques (2-6 \%) [117].

Dans la littérature, plus de $80 \%$ des adénocarcinomes nasosinusiens de type intestinal expriment la protéine CDX2 [22][69].

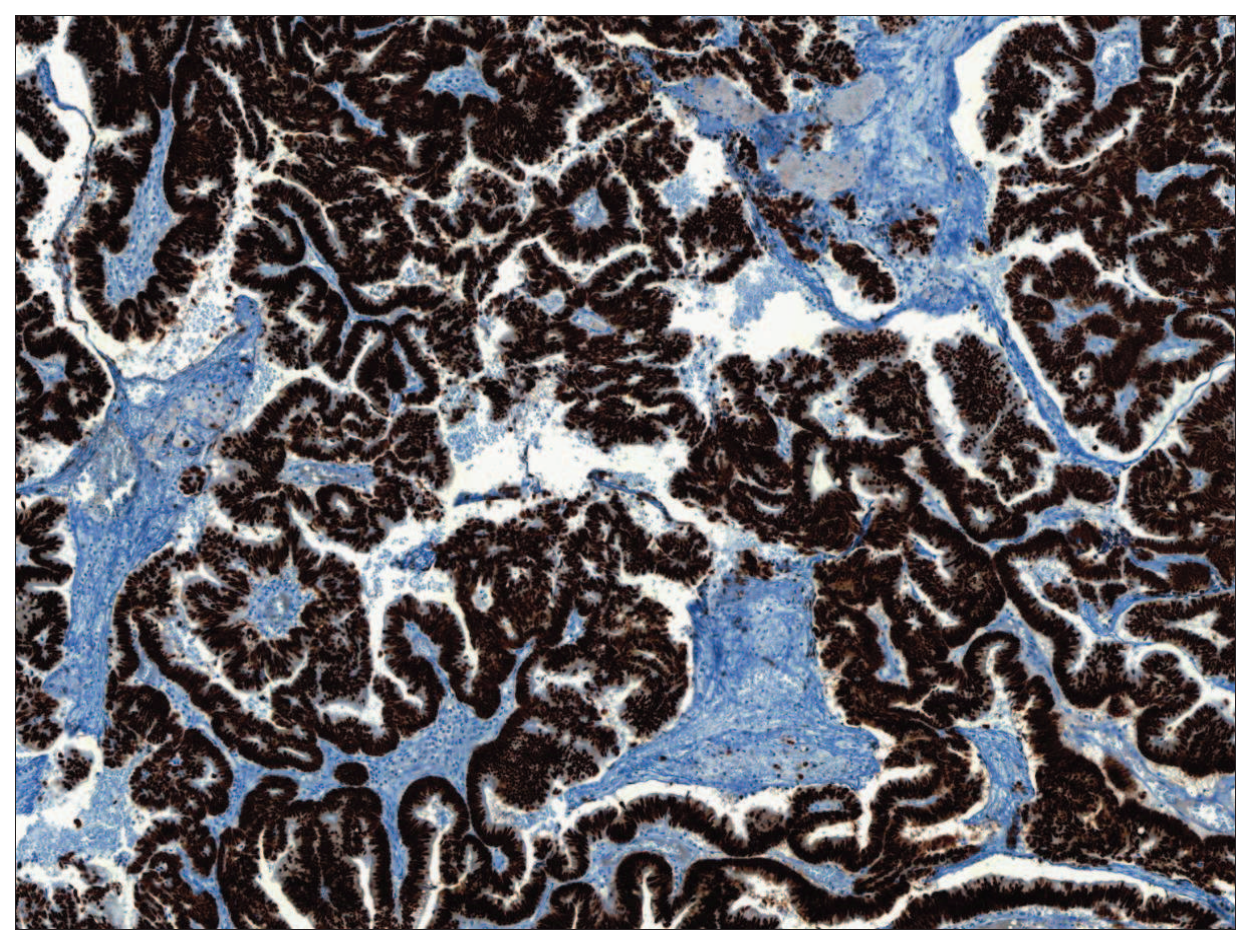

Figure $25_{\mathrm{A}}$ :

Immunomarquage CDX2 d'un ITAC

(marquage nucléaire).

\section{$B$ - Les cytokératines 7 (CK 7) et 20 (CK 20)}

Les cytokératines sont les constituants des filaments intermédiaires de kératine (d'un diamètre d'environ $10 \mathrm{~nm}$ ), que l'on retrouve spécifiquement dans les tissus épithéliaux. Elles sont classées de 1 à 20 en fonction de leur poids moléculaire (40 à $67 \mathrm{kD}$ ) et comprennent 12 kératines de type I (CK9 à 20), acides et 8 de type II (CK1 à 8), neutres-basiques. 
Lors du développement cellulaire les kératines sont associées en tétramère : 2 molécules de kératines de type I sont associées à 2 molécules de kératine de type II. Les kératines de type I sont codées par 2 gènes situés sur le chromosome 17, celles de types II par des gènes situés sur le chromosome 12. Une cellule peut produire de multiples types de kératine. Du fait de leur localisation spécifique dans les tissus épithéliaux, elles sont utiles dans l'identification de l'origine épithéliale d'une tumeur.

La CK 7, kératine de bas poids moléculaire de 54 kD, de type II, est exprimée dans de nombreux épithéliums simples, canalaires, pseudostratifiés et par les cellules mésothéliales. La majorité des adénocarcinomes nasosinusiens de type non intestinal exprime cet anticorps, alors que les ITAC restent négatifs dans 43 à $90 \%$ des cas, selon les séries [69].

La CK 20, kératine de bas poids moléculaire de $46 \mathrm{kDa}$, appartient au groupe des cytokératines de type I.

Une expression de la CK 20 est généralement retrouvée dans la plupart des adénocarcinomes coliques, des tumeurs mucineuses de l'ovaire et des carcinomes à cellules transitionnelles ou à cellules de Merkel [118-119].

70 à $98 \%$ des adénocarcinomes nasosinusiens de type intestinal expriment cette protéine, le marquage étant cytoplasmique, à renforcement membranaire [22].

Une positivité a également été soulignée au niveau des adénocarcinomes gastriques (50\%), du système biliaire $(75 \%)$ et pancréatique (40\%).

Par ailleurs, moins de $2 \%$ des cas d'adénocarcinomes mammaires ou pulmonaires et des carcinomes épidermoïdes expriment cette cytokératine. 
Dans de très rares cas d'adénocarcinomes ovariens (autres que mucineux) ou endométriaux et de carcinomes rénaux ou à petites cellules, il a été retrouvé une expression faible et focal de cet antigène par les cellules tumorales.

Cette étude a été réalisée dans le but de déterminer la proportion de lésions tumorales exprimant le CDX 2 et/ou la CK 20 et/ ou la CK 7. Elle a également permis d'évaluer l'intérêt de ces immunomarquages comme " outil diagnostic », aidant à la distinction des différents sous-types histologiques d'adénocarcinomes nasosinusiens.

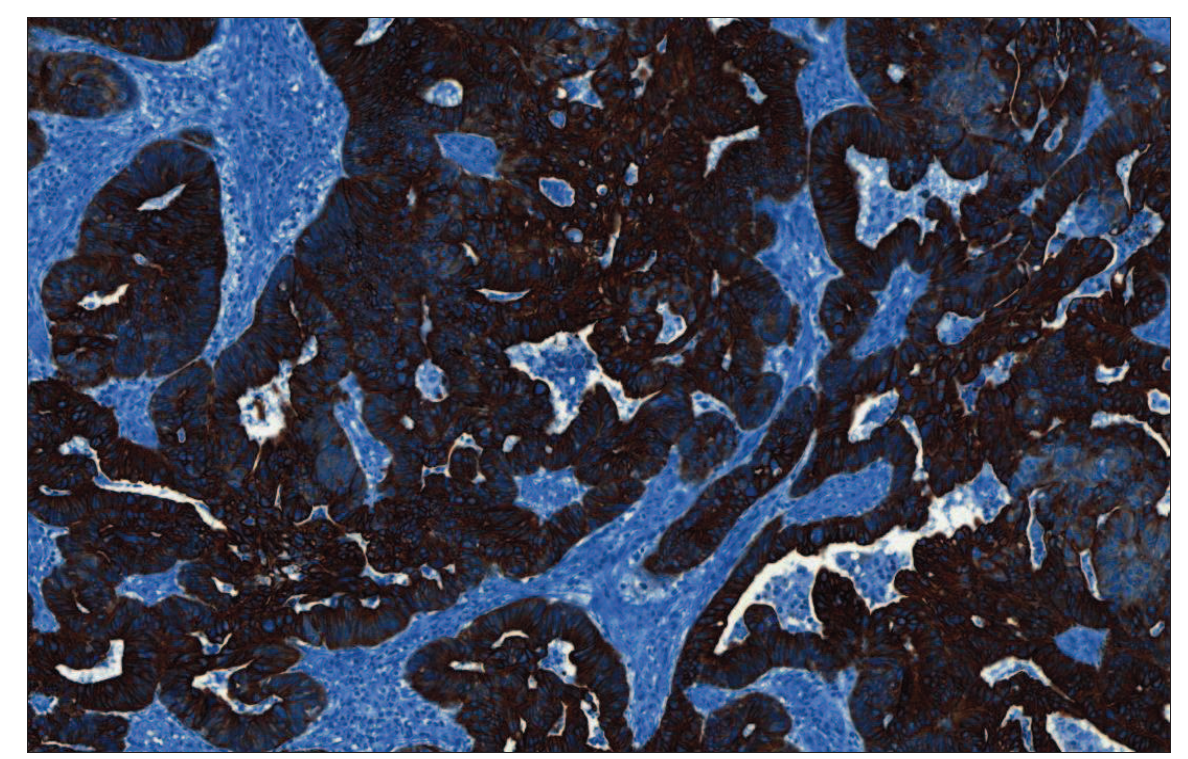

Figure $25_{B}$ : Immunomarquage CK20 d'un ITAC

(marquage cytoplasmique).

\section{C - Le récepteur tyrosine kinase EGFR}

Cette analyse immunohistochimique a été effectuée afin d'évaluer le niveau d'expression de la protéine EGFR par les cellules tumorales et, de ce fait, d'évaluer le potentiel de réponse thérapeutique aux «anti-EGFR ». Le type de marquage cellulaire est membranaire. II est positif au sein de la muqueuse respiratoire normale, celle-ci servant de témoin positif interne. 


\section{III - Etude en biologie moléculaire des gènes EGFR et KRAS}

\section{1 - Extraction d'ADN génomique}

Cette étape pré-analytique nécessite un contrôle morphologique effectué par un anatomo-pathologiste sur la lame colorée à l'Hématéine-Eosine-Safran ayant servi à établir le diagnostic. Ce contrôle permet de vérifier la nature du prélèvement et de repérer une zone où le pourcentage de cellules tumorales est en adéquation avec le seuil de sensibilité de l'analyse moléculaire: un minimum de $20 \%$ de cellules tumorales est considéré comme nécessaire pour la validité du test.

4 coupes de $10 \mu \mathrm{m}$ sont effectuées à partir du bloc de paraffine correspondant à la lame examinée par le pathologiste. La zone tumorale d'intérêt est ensuite macrodisséquée au bistouri par apposition des lames blanches sur la lame colorée et I'ADN génomique tumoral est extrait à l'aide du kit «RecoverAll ${ }^{\mathrm{TM}}$ Total Nucleic Acid Isolation Kit for FFPE Tissues» (Applied Biosystems) selon les recommandations du fournisseur.

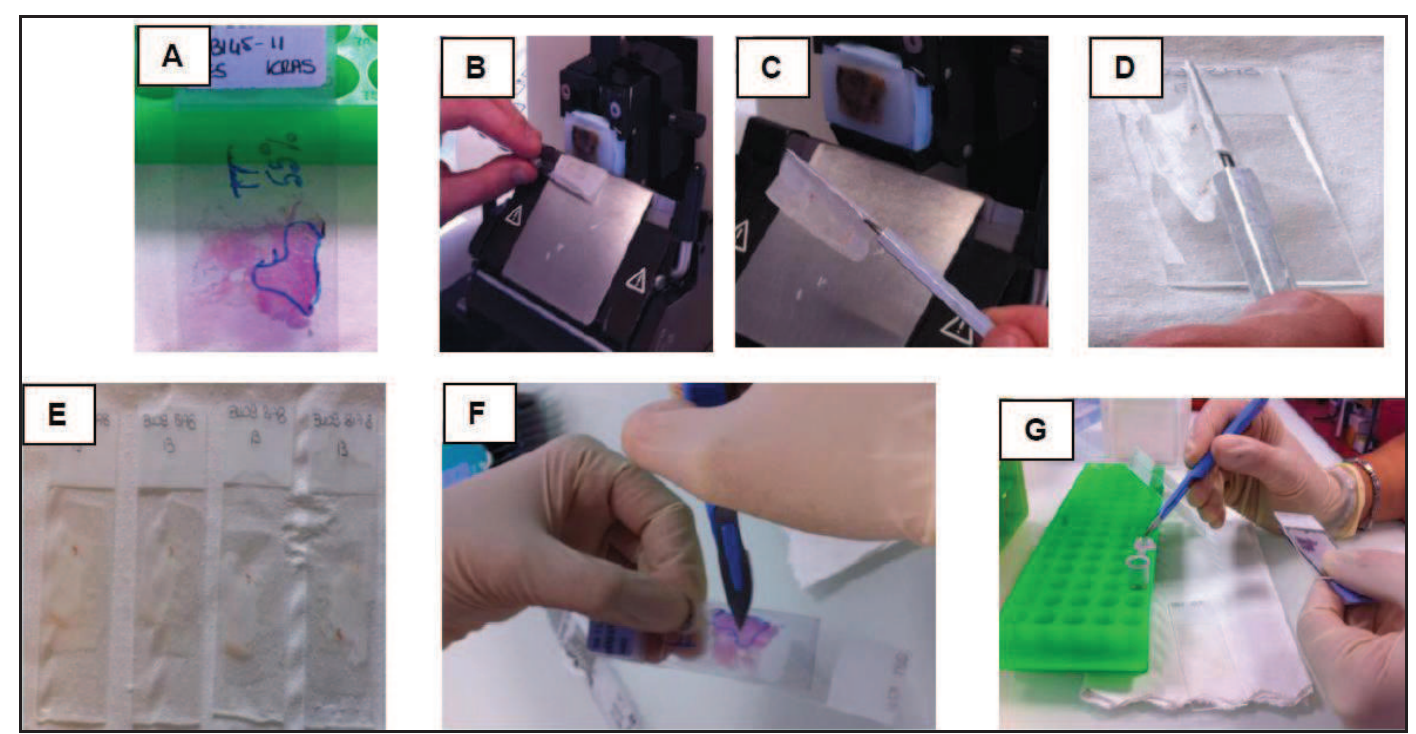

Figure 26 (extrait de la thèse de Solène Houlle)

A : Sélection de la zone tumorale d'intérêt - B, C, D, E : Elaboration de 4 coupes du bloc de paraffine correspondant à la lame sélectionnée $-\mathrm{F}$ : Macrodissection de la zone tumorale d'intérêt à partir des 4 coupes en s'aidant de la lame initiale - G : Dépôt dans un microtube du tissu tumoral macrodisséqué afin d'en extraire l'ADN. 


\section{2 - Génotypage EGFR et KRAS}

Cette étude est réalisée par :

- SNaPshot $\circledR$ multiplex sur les produits d'amplifications:

- De l'exon 2 (codons 12 et 13) de KRAS.

- Des exons 18, 20 et 21 de l' EGFR.

- Analyse des fragments d'amplification des exons 19 et 20 de l'EGFR.

A - SNaPshot $($ multiplex

La détection des mutations ponctuelles des codons 12 et 13 (c.34 G>A, c.34G>C, c.34G>T, с.35G>A, с.35G>C, с.35G>T, с.37G>A, c.37G>C, с.37G>T, с.38 G>A) de l'exon 2 du gène $K R A S$ et des exons 18 (c.2155G et c.2156G), 20 (c.2369C) et 21 (c.2573T et c.2582T) du gène EGFR se fait en plusieurs étapes :

- Amplification multiplexe des régions d'intérêts par PCR, à partir d'amorces spécifiques de:

- l'exon 18 de l'EGFR : 5' - CCCCCCCAGCTTGTGG - 3' (EGFR 18-F) et 5' - ACCGTGCCGAACGCAC - 3' (EGFR 18-R).

- l'exon 20 de l'EGFR : 5' - CTCTCCCTCCCTCCAGGAAG - 3' (EGFR 20-F) et 5' - TTCCCGGACATAGTCCAGGA - 3' (EGFR 20-R).

- l'exon 21 de l'EGFR : 5' - CGCAGCATGTCAAGATCACAG - 3' (EGFR 21-F) et 5' - TGGCTGACCTAAAGCCACCT - 3' (EGFR 21-R).

- l'exon 2 de KRAS : 5' - AAGGCCTGCTGAAAATGACTG - 3' (KRAS-F) et 5' - CAAAGAAATGGTCCTGCACCAG - 3' (KRAS-R). 
L'amplification est réalisée dans un volume final de $25 \mu$, comprenant 500 ng d'ADN génomique tumoral, $1 \mathrm{x}$ du tampon de PCR incluant la Taq DNA polymérase et les dNTPs (Qiagen multiplex PCR master mix, Qiagen) et 0,2 $\mu \mathrm{M}$ de chaque amorce. Après une étape de dénaturation de l'ADN et d'activation de la Taq DNA polymérase 15 minutes à $95^{\circ} \mathrm{C}, 45$ cycles de $\mathrm{PCR}$ de 30 secondes à $94^{\circ} \mathrm{C}, 90$ secondes à $63^{\circ} \mathrm{C}$ et 90 secondes à $72^{\circ} \mathrm{C}$ suivis d'une élongation finale de 10 minutes à $72^{\circ} \mathrm{C}$ sont réalisés.

\section{Figure 27}

Amplification d'une région contenant les codons 12 et 13 de KRAS à partir d'un couple d'amorces sens et antisens
GGT GGC CCACCG

\section{GAT GGC} CTA CCG

- Purification des produits de PCR. Cette étape permet d'éliminer les amorces et les dNTPs résiduels. Les produits de PCR sont séparées en fonction de leurs poids moléculaire par électrophorèse sur un gel d'agarose à $3 \%$ en présence de bromure d'éthidium (BET). Les bandes d'agarose contenant les produits de PCR sont ensuite découpées et les produits de PCRs en sont extrait, à l'aide du kit d'extraction NucleoSpin Extract II (Machery-Nagel) selon les recommandations du fournisseur.

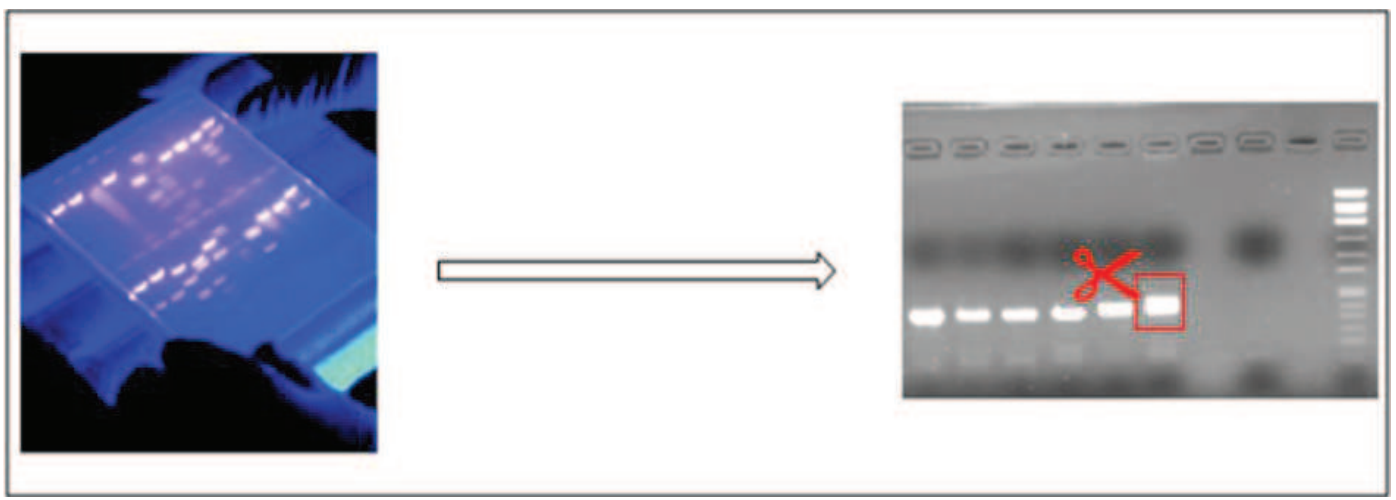

Figure 28:

Découpage du produit de PCR après sa migration sur gel d'agarose $3 \%-B E T$. 


\section{- Réaction d'extension d'amorces SNaPshot@}

Le principe de cette méthode est basé sur l'incorporation de didésoxyribonucléotides (ddNTPs) fluorescents à partir d'amorces hybridées par leur extrémité 3' sur les nucléotides précédents les nucléotides d'intérêts. Les ddNTPs incorporés sont complémentaires des nucléotides d'intérêt et provoquent l'arrêt de l'élongation. Le type de ddNTP incorporé est caractérisé par sa fluorescence.

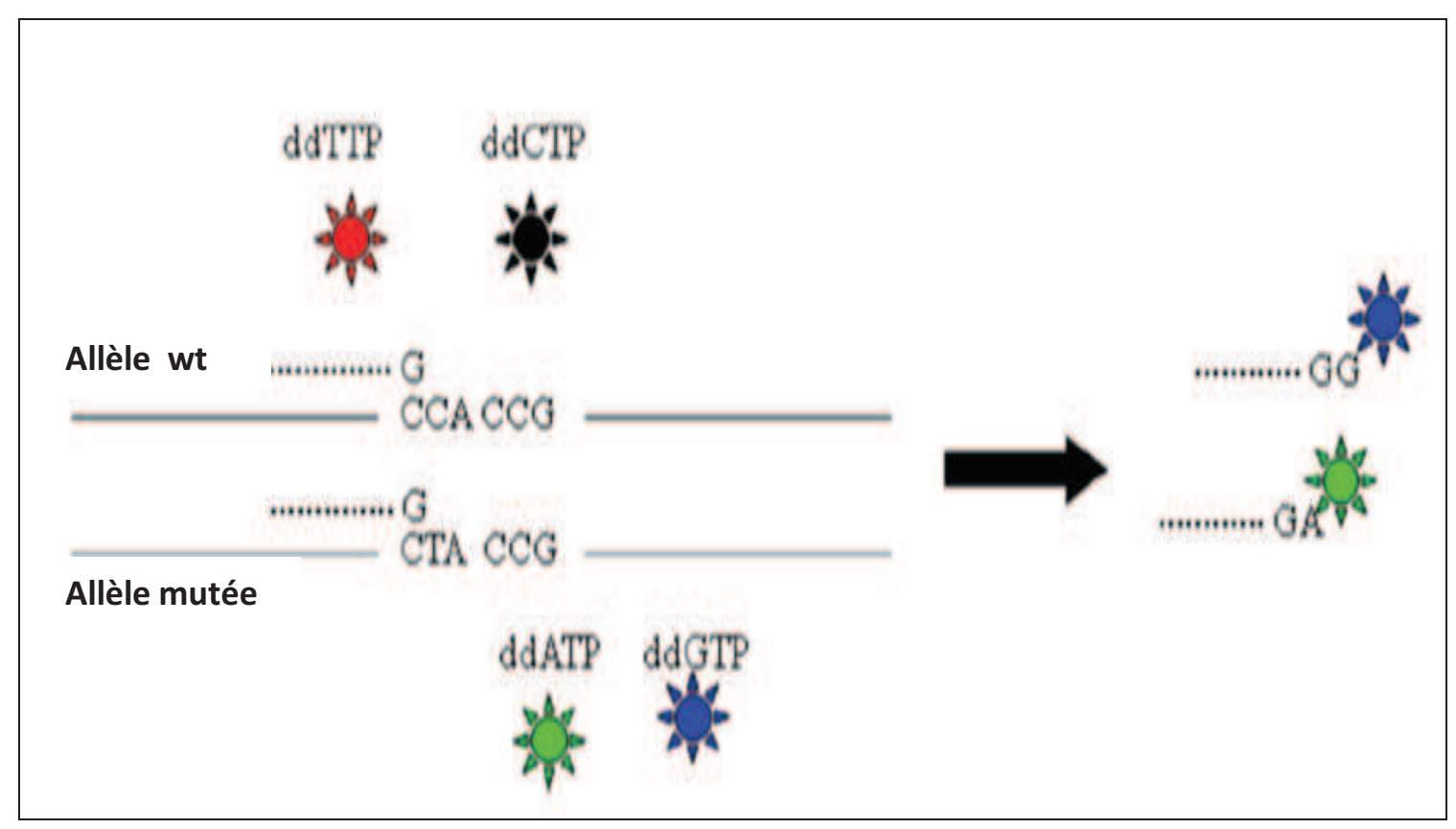

Figure 29:

Principe du SNaPshot ${ }^{\circ}$ / Exemple : mutation KRAS c.35G>A

Des amorces spécifiques par leur extrémité 3' du nucléotide précédent le nucléotide d'intérêt s'hybrident sur les allèles sauvage et mutant. Ces amorces sont étendues par un ddNTP marqué par un fluorochrome spécifique complémentaire de la base sauvage (ddGTP) ou de la base mutante (ddATP). Dans le cas d'une mutation hétérozygote, deux types d'amorces étendues sont obtenues. 
Les amorces de SNaPshot $\Re$, détaillées dans le tableau 5, ont été choisies sur les brins sens ou anti-sens et sont spécifiques par leurs extrémités 3' des nucléotides situés en amont des nucléotides EGFR c.2155G, EGFR c.2156G, EGFR c.2369C, EGFR c.2573T, EGFR c.2582T, KRAS c.34G, KRAS c.35G, KRAS c.37G et KRAS c.38G. Elles sont additionnées au niveau de leurs extrémités $5^{\prime}$ de motifs oligonucléotidiques de taille variable et non complémentaires du génome humain afin de leurs conférer un poids moléculaire spécifique qui permettra la détection simultanée des amorces étendues.

Tableau 5 : Détail des amorces de SNaPshot ${ }^{\circledR}$

\begin{tabular}{|c|c|c|c|}
\hline $\begin{array}{c}\text { Position } \\
\text { du } \\
\text { nucléotide } \\
\text { étendu }\end{array}$ & Séquence & Brin & $\begin{array}{l}{[\mathrm{c}]} \\
\mu \mathrm{M}\end{array}$ \\
\hline $\begin{array}{l}\text { EGFR } \\
\mathrm{c} .2155\end{array}$ & 5'-TGGTTAGATGGAACGCACCGGAGC-3' & antisens & 0.05 \\
\hline $\begin{array}{l}E G F R \\
c .2156\end{array}$ & 5'-TAGATG TGGTTAGATGCGAACGCACCGGAG-3' & antisens & 0.09 \\
\hline $\begin{array}{l}\text { EGFR } \\
\text { c.2369 }\end{array}$ & 5'-TGGTTAGATG TGGTTAGATG GAAGGGCATGAGCTGC-3' & antisens & 0.01 \\
\hline $\begin{array}{l}E G F R \\
\text { c. } 2573\end{array}$ & 5'-ATG TGGTTAGATGTGGTTAGATG AAGATCACAGATTTTGGGC-3' & sens & 0.015 \\
\hline $\begin{array}{l}\text { EGFR } \\
\text { c.2582 }\end{array}$ & 5'-GATGTGGTTAGATGTGGTTAGATGTGGTTAGATGTGGGCTGGCCAAAC-3' & sens & 0.02 \\
\hline $\begin{array}{c}\text { KRAS } \\
\text { c.34 }\end{array}$ & $\begin{array}{l}\text { 5' - TTAGATG TGGTTAGATGTGGTTAGATG TGGTTAGATGACTCTTGCCTACGCCAC - } \\
\text { 3' }\end{array}$ & antisens & 0.03 \\
\hline $\begin{array}{c}\text { KRAS } \\
\text { c.35 }\end{array}$ & $\begin{array}{l}\text { 5' - } \\
\text { ATGTGGTTAGATGTGGTTAGATGTGGTTAGATGTGGTTAGATGCACTCTTGCCTACGCCA } \\
\text {-3' }\end{array}$ & antisens & 0.03 \\
\hline $\begin{array}{l}\text { KRAS } \\
\text { c.37 }\end{array}$ & $\begin{array}{l}\text { 5' - GGTTAGATG } \\
\text { TGGTTAGATGTGGTTAGATGTGGTTAGATGTGGTTAGATGGGCACTCTTGCCTACGC - 3' }\end{array}$ & antisens & 0.04 \\
\hline $\begin{array}{c}\text { KRAS } \\
\text { c.38 }\end{array}$ & $\begin{array}{l}\text { 5' - AGATG TGGTTAGATGTGGTTAGATGTGGTTAGATGTGGTTAGATGTGGTTAGATG } \\
\text { AGGCACTCTTGCCTACG - 3' }\end{array}$ & antisens & 0.09 \\
\hline
\end{tabular}

La réaction d'extension d'amorces est réalisée dans un volume final de $10 \mu \mathrm{l}$ comprenant , 1x du tampon de réaction (SNaPshot ${ }^{\circ}$ Multiplex ready reaction mix, Applied Biosystems) incluant les ddNTPs marqués par des fluorochromes, les neufs amorces de SNaPshot ${ }^{\circledR}$ (aux concentrations indiquées dans le tableau 5) et $2 \mu$ des produits de PCR purifiés. 25 cycles de 10 secondes à $96^{\circ} \mathrm{C}, 5$ secondes à $50^{\circ} \mathrm{C}$ et 30 secondes à $60^{\circ} \mathrm{C}$ sont ensuite réalisés. 


\section{- Purification du produit de SNaPshot ${ }^{\circledR}$}

Cette étape permet de déphosphoryler les ddNTPs résiduels. Les produits de SNaPshot ${ }^{\circ}$ sont traités une heure à $37^{\circ} \mathrm{C}$ par une unité de phosphatase alcaline (Shrimp alkaline phosphatase, GE Healthcare), puis l'enzyme est inactivée 15 minutes à $37^{\circ} \mathrm{C}$.

\section{- Migration sur séquenceur automatique}

Les amorces étendues sont séparées par électrophorèse capillaire sur un séquenceur (ABI PRISM 3130xI).

\section{- Analyse des résultats}

Cette étape est réalisée à l'aide du logiciel GeneMapper version 4.0 (Applied Biosystems).

La présence d'une mutation hétérozygote se traduit par la détection pour une position étudiée de deux ddNTPs incorporés : I'un complémentaire du nucléotide sauvage et l'autre du nucléotide muté. 


\section{ANALYSE DES RESULTATS DE SNaPshot®}
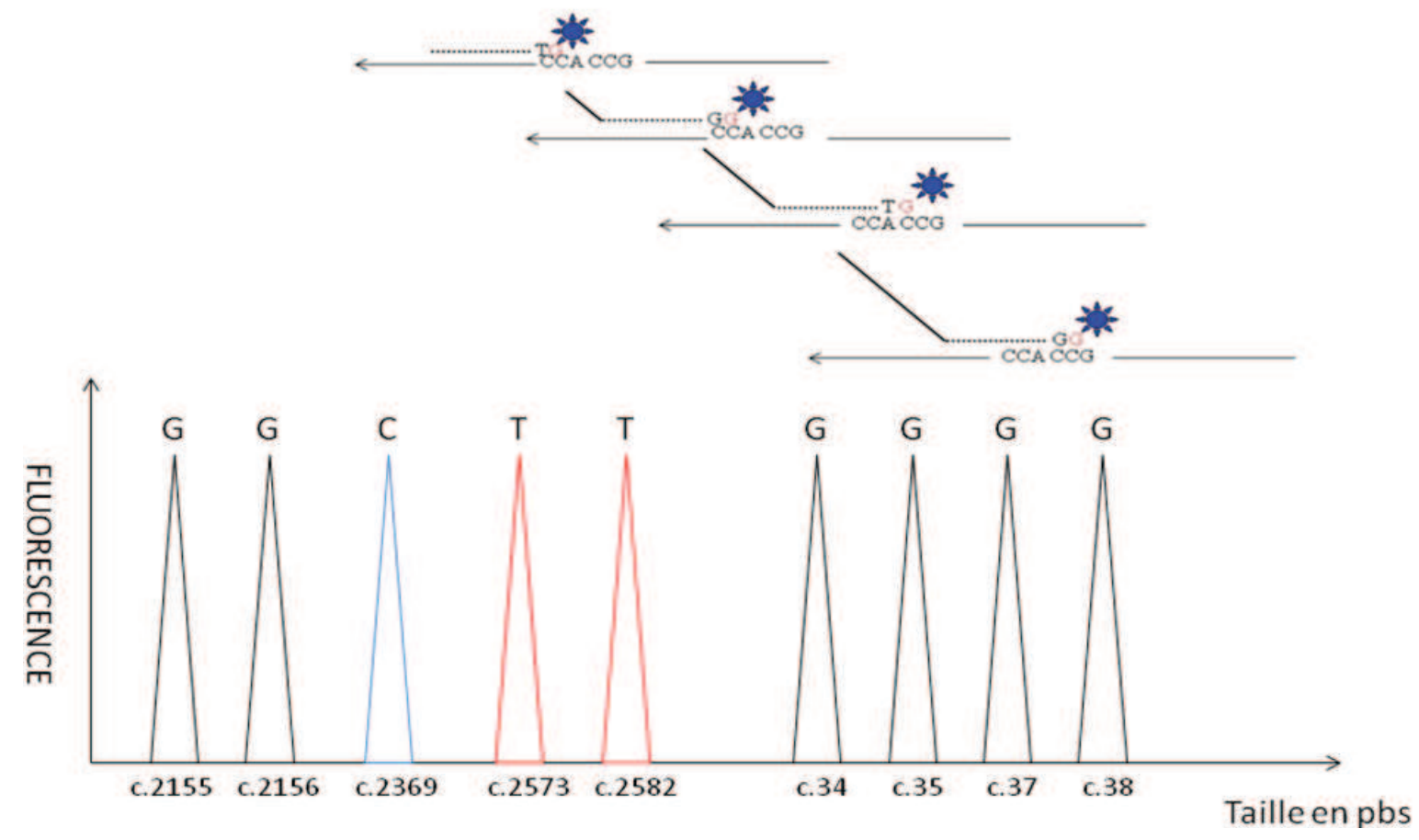

Figure 30 : Lecture simultanée des nucléotides d'intérêts d'EGFR et KRAS.
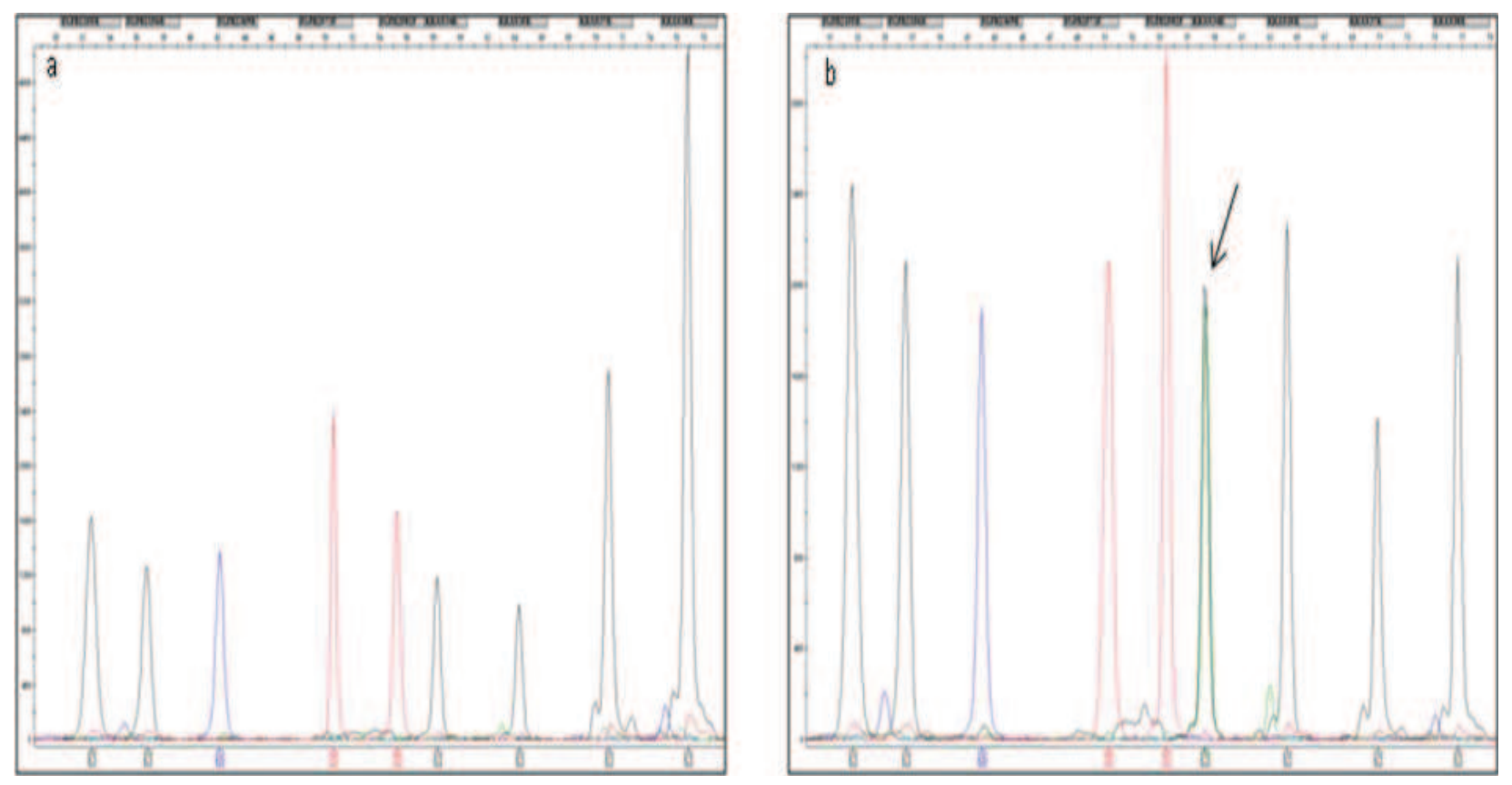

Figure 31: Exemple d'un profil SNaPshot $\circledast$ sauvage (a) et muté (b) KRAS. 
$\mathrm{B}-$ Analyse des fragments d'amplification des exons 19 et 20 de l'EGFR

Cette technique vise à identifier :

- Les délétions en phase de l'exon 19 (4 à 6 acides aminés), induisant une sensibilité aux TKI.

- Les insertions / duplications en phase de l'exon 20, induisant plutôt une résistance primaire aux TKI.

L'analyse de fragments se fait en plusieurs étapes:

- Amplification multiplexe des exons 19 et 20 de l'EGFR, par PCR, à partir d'amorces spécifiques :

- Exon 19 : 5' - AACGTCTTCCTTCTCTCTGTCATAG - 3' (EGFR 19-F) et 5' - GCAAAGCAGAAACTCACATCG - 3' (EGFR 19-R).

- Exon 20 : 5' - CCACACTGACGTGCCTCTC - 3' (EGFR 20-F) et 5' - GCTGCACGGTGGAGGTG - 3' (EGFR 20-R).

L'amplification est réalisée dans un volume final de $12,5 \mu \mathrm{l}$ comprenant $500 \mathrm{ng}$ d'ADN, $1 \mathrm{x}$ de tampon de PCR, incluant la Taq DNA polymérase et les dNTPs (Qiagen multiplex PCR master mix, Qiagen), $2 \mu \mathrm{M}$ des amorces sens et antisens spécifiques de l'exon 19 d'EGFR et $1 \mu \mathrm{M}$ des amorces sens et antisens spécifiques de l'exon 20.

Après une étape de dénaturation de l'ADN et d'activation de la Taq DNA polymérase 15 minutes à $95^{\circ} \mathrm{C}, 45$ cycles de $\mathrm{PCR}$ de 30 secondes à $94^{\circ} \mathrm{C}, 90$ secondes à $59^{\circ} \mathrm{C}$ et 90 secondes à $72^{\circ} \mathrm{C}$ suivis d'une étape d'élongation finale de 10 minutes à $72^{\circ} \mathrm{C}$ sont réalisés. 


\section{- Séparation des produits de PCR}

Une des amorces de chaque couple est marquée à son extrémité 5' par un fluorochrome spécifique, ce qui permet de visualiser les produits de PCR après séparation par électrophorèse capillaire sur séquenceur AB 3130xl.

\section{- Analyse des résultats}

Cette étape est réalisée grâce au logiciel GeneMapper version 5.3.1 (Applied Biosystems).

Afin de valider la présence d'une mutation ou d'un réarrangement génique, deux analyses indépendantes ont été réalisées.

\section{IV - Analyse statistique}

Les différentes corrélations anatomo-cliniques ont été évaluées par le test de FISHER, étant une alternative au test $\mathrm{du} \mathrm{X}^{2}$ lorsque les échantillons comportent un faible effectif.

Les courbes de survie ont été estimées par la méthode de Kaplan Meier et comparées par le test du Log-rang. L'analyse multi variée des facteurs prédictifs de survie a été réalisée en utilisant un modèle de Cox avec calcul du Hazard Ratio (HR) et de l'intervalle de confiance (IC) à $95 \%$. Un « $p \leq 0,05$ » était considéré comme statistiquement significatif.

Les calculs statistiques ont été réalisés en utilisant les logiciels MedCalc et Sas 9-3. 
ANALYSE DES RESULTATS DES FRAGMENTS D'AMPLIFICATION

DES EXONS 19 ET 20 DE L'EGFR

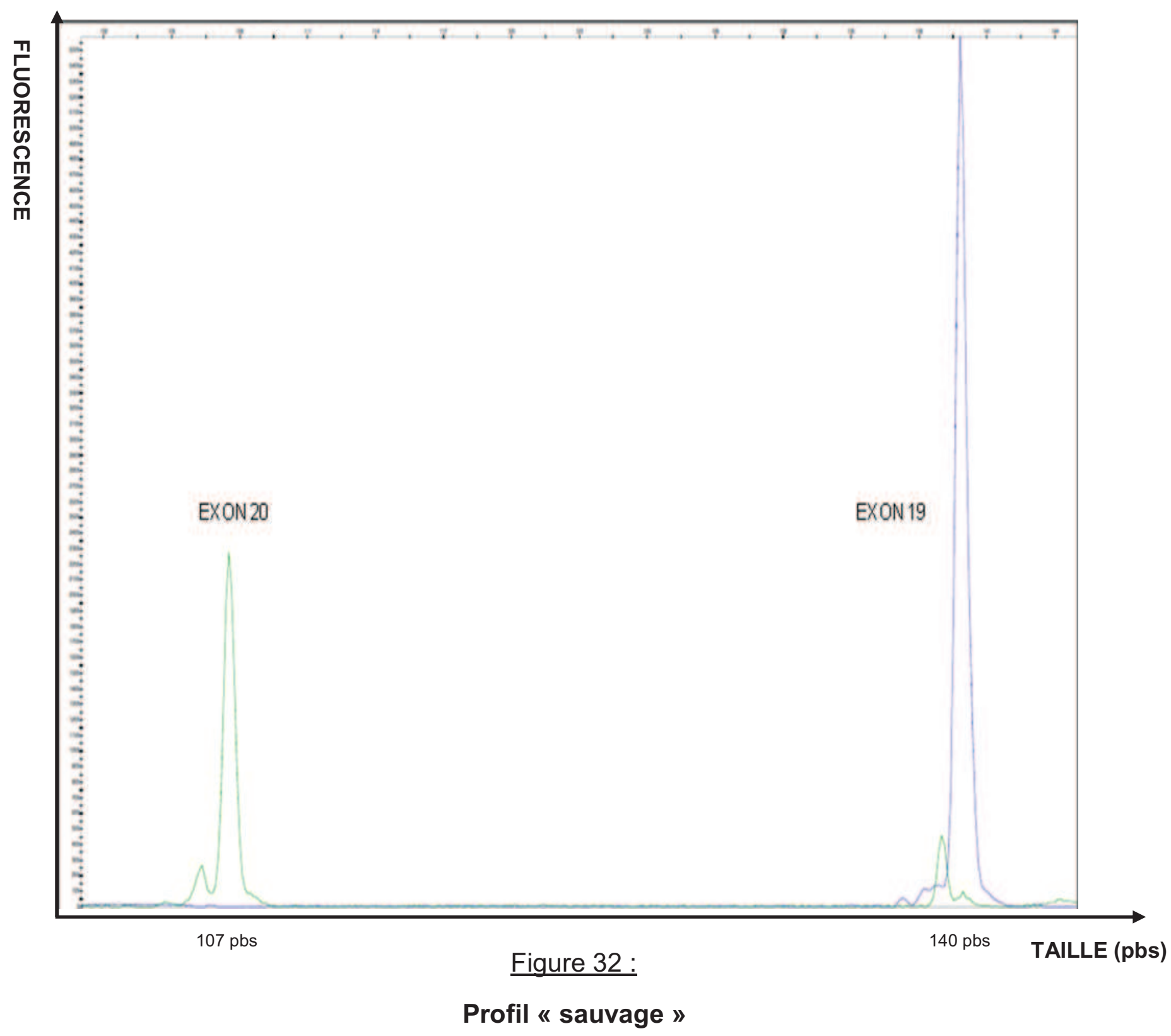

Les deux fragments retrouvés sont de taille attendue (exon $19: 140$ pbs et exon $20: 107$ pbs).

Le profil est dit « sauvage » ou « wild type », sans délétion ni insertion. 


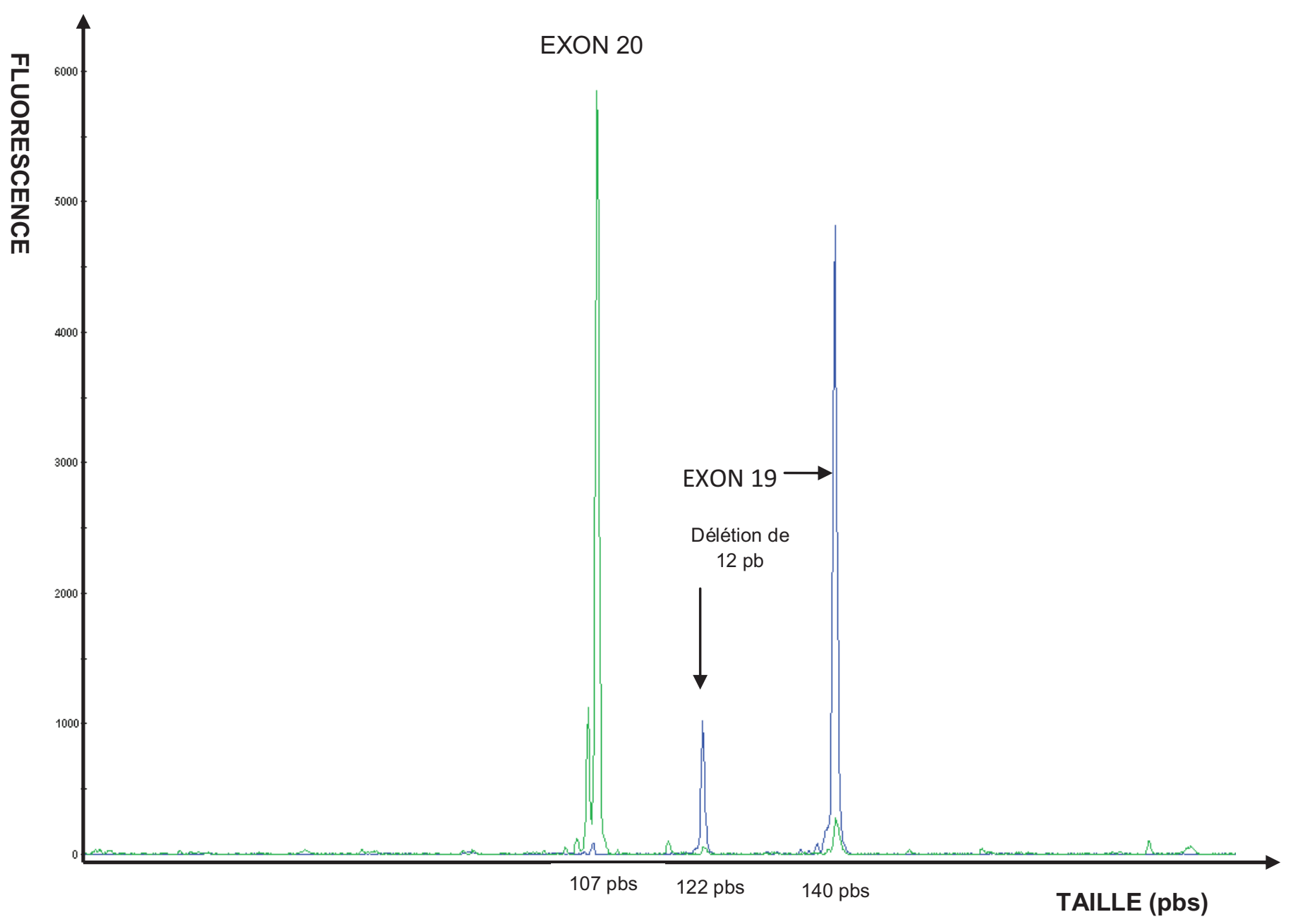

Figure 33:

Profil correspondant à la détection d'une « délétion de l'exon 19 »

Le fragment correspondant à l'exon 20 est de taille attendue (107 pbs).

Pour l'exon 19, il est retrouvé un fragment de taille attendue (140 pbs) et un fragment plus court à $122 \mathrm{pbs}$. Ce dernier fragment correspond à l'amplification d'un exon 19 ayant perdu 12 pbs. 


\section{I - Caractéristiques cliniques}

Les principales données anatomo-cliniques intéressant les 38 patients inclus dans notre étude sont résumées dans le tableau 6.

\section{1 - Epidémiologie}

II s'agit d'une série d'adénocarcinomes des fosses nasales et des sinus paranasaux diagnostiqués entre janvier 1983 et octobre 2010. Pour ces 38 patients, nous avons disposé de 54 prélèvements, correspondant aux biopsies initiales et/ou pièces opératoires et aux éventuelles récidives.

Les 38 patients inclus dans cette étude étaient répartis de la façon suivante : 35 hommes et 3 femmes, soit un sexe ratio de 11,7H/1F, âgés de 41 à 86 ans au moment du diagnostic, avec une moyenne de 64 ans.

Une notion d'exposition à un facteur de risque était retrouvée chez 31 des 38 patients (dont 30 au bois et 1 au cuir), soit dans $81,6 \%$ des cas. La durée d'exposition était précisée dans 24 des 38 cas (63\%) et était en moyenne de 27 ans, variant de 3 à 49 ans selon les sujets.

La notion d'exposition à un autre facteur de risque incriminé dans ce type de tumeur, tels que le nickel ou le chrome n'a été retrouvée dans aucun des cas. 
Tableau 6 données cliniques

\begin{tabular}{|l|l|l|l|l|l|}
\hline & & & & & \\
\hline & & & & & \\
\hline & & & & & \\
\hline
\end{tabular}




\section{2 - Signes clinico-radiologiques et classification TNM}

Les plaintes fonctionnelles (tableau 7) les plus fréquemment rapportées lors du diagnostic initial étaient : une obstruction nasale dans 26 cas $(68,4 \%)$, des épistaxis dans 18 cas $(47,4 \%)$, une rhinorrhée et/ou une exophtalmie dans 7 cas (18,4\%), des douleurs et/ou une tumeur visible dans 6 cas $(15,8 \%)$, une diplopie dans 4 cas $(10,5 \%)$ et des adénopathies dans 1 cas $(2,63 \%)$. Ces troubles étaient unilatéraux dans 35 cas (92\%). L'association de plusieurs symptômes pouvaient évidemment être présente chez un même patient.

Avant la première consultation, un délai supérieur à 6 mois était observé pour la moitié des patients.

Tableau 7 : Signes fonctionnels au moment du diagnostic

\begin{tabular}{|c|c|c|}
\hline Signes fonctionnels & Nombre de patients & $\%$ \\
\hline Obstruction nasale & 26 & 68,4 \\
\hline Epistaxis & 18 & 47,4 \\
\hline Rhinorrhée & 7 & 18,4 \\
\hline Exophtalmie & 7 & 18,4 \\
\hline Douleur & 6 & 15,8 \\
\hline Tumeur visible & 6 & 15,8 \\
\hline Diplopie & 4 & 10,5 \\
\hline Adénopathies & 1 & 2,63 \\
\hline Amaigrissement & 0 & 0 \\
\hline
\end{tabular}

En ce qui concerne la localisation tumorale, $81 \%$ des tumeurs se développaient au niveau des sinus ethmoïdaux. 10 lésions siégeaient à droite, 18 à gauche et 3 étaient bilatérales. Pour 7 cas, la localisation tumorale n'était pas précisée. 
Une atteinte de la paroi de l'orbite était constatée, au moment du diagnostic, chez 10 patients $(26,3 \%)$, un envahissement de la base du crâne chez 4 patients $(10,5 \%)$ ou méningé chez 10 patients $(26,3 \%)$.

La répartition des 38 cas selon la classification clinico-radiologique UICC 2010 est décrite dans le tableau 8.

Tableau 8 : Répartition des 38 cas selon l'UICC 2010.

\begin{tabular}{|c|c|c|c|c|c|c|c|c|c|c|c|c|}
\hline \multicolumn{2}{|c|}{$T$} & \multicolumn{9}{c|}{$N$} & \multicolumn{3}{c|}{ M } \\
\hline$T_{1}$ & $T_{2}$ & $T_{3}$ & $T_{4}{ }^{*}$ & $N_{0}$ & $N_{1}$ & $N_{2} \mathbf{a}$ & $N_{2} b$ & $N_{2} \mathbf{C}$ & $\mathbf{N}_{3}$ & $\mathbf{M}_{0}$ & $\mathbf{M}_{1}$ & $\mathbf{M}_{\mathbf{x}}$ \\
\hline 1 cas & 15 cas & 6 cas & 16 cas & 37 cas & 0 cas & 0 cas & 1 cas & 0 cas & 0 cas & 38 cas & 0 cas & 0 cas \\
$2,63 \%$ & $39,5 \%$ & $15,8 \%$ & $42 \%$ & $97,4 \%$ & $0 \%$ & $0 \%$ & $2,6 \%$ & $0 \%$ & $0 \%$ & $100 \%$ & $0 \%$ & $0 \%$ \\
\hline
\end{tabular}

${ }^{*}$ Dont $4 \mathrm{~T}_{4 \mathrm{a}}$ et $12 \mathrm{~T}_{4 \mathrm{~b}}$

\section{3 - $\underline{\text { Traitements }}$}

Un protocole thérapeutique " curatif » a été proposé pour 36 patients (95\%), toujours représenté par un geste chirurgical, accompagné, pour 34 d'entre eux d'une radiothérapie.

Deux patients $(5 \%)$ ont bénéficié d'un traitement « palliatif », par radio-chimiothérapie. 


\section{4 - Récidives et survie}

La durée du suivi des 38 patients a varié de 6 à 279 mois, avec une moyenne de 61 mois.

Une récidive tumorale locale est survenue dans 12 cas (33,6\%), dans un délai de 5 à 156 mois, avec une moyenne de 30,25 mois. Parmi ces lésions récidivantes, une avait été classées initialement $T_{1}$, deux $T_{2}$, une $T_{3}$ et huit $T_{4}$. Les traitements alors effectués ont comporté de façon plus ou moins combinée, un geste chirurgical dans 6 cas, une radiothérapie dans 3 cas, une chimiothérapie dans 3 cas. Une simple surveillance a été proposée pour 3 cas.

Deux cas $(5,3 \%)$, correspondant respectivement à une tumeur initialement classée $T_{4} N_{0} M_{0}$ et $T_{4} N_{2 b} M_{0}$, ont présenté une métastase ganglionnaire, dans un délai de 6 à 82 mois, traitée alors par radiothérapie et/ou chimiothérapie.

Six cas (16\%) ont développé une métastase à distance dans un délai de 6 à 36 mois, avec une moyenne de 19,5 mois. Deux de ces cas présentaient initialement une tumeur classée $T_{4}$, un $T_{3}$, deux $T_{2}$ et un $T_{1}$. Le siège de ces lésions métastatiques étaient : pulmonaire ( 1 cas), rachidien ( 1 cas), cérébral ( 2 cas) ou non précisé ( 2 cas).

Un décès imputable à la progression tumorale a été retrouvée dans 12 cas $(31,6 \%)$, par extension locorégionale dans 8 cas $(21 \%)$, et dans un tableau de diffusion métastatique pour 4 cas $(10,5 \%)$. Parmi ces 12 observations, une lésion tumorale primitive était $T_{1}$, une était $T_{2}$, deux étaient $T_{3}$ et 8 étaient $T_{4}$. 
Ceci permet d'établir un taux de mortalité selon les différents stades TNM suivant :

$\mathrm{T}_{1}$ et $\mathrm{T}_{2}(16$ cas $)$ 2 décès, soit un taux de mortalité de $12,5 \%$

$\mathrm{T}_{3}$ et $\mathrm{T}_{4}(22$ cas $)$ 10 décès, soit un taux de mortalité de $45 \%$.

Chez 14 patients (37\%), aucun argument en faveur d'une maladie tumorale évolutive n'était retrouvé lors de leur dernière consultation.

Vingt-deux cas étaient considérés comme guéris, soit un taux de survie globale à 3 ans estimé à $56 \%$, IC 95\% [37,2\%; 69,6\%].

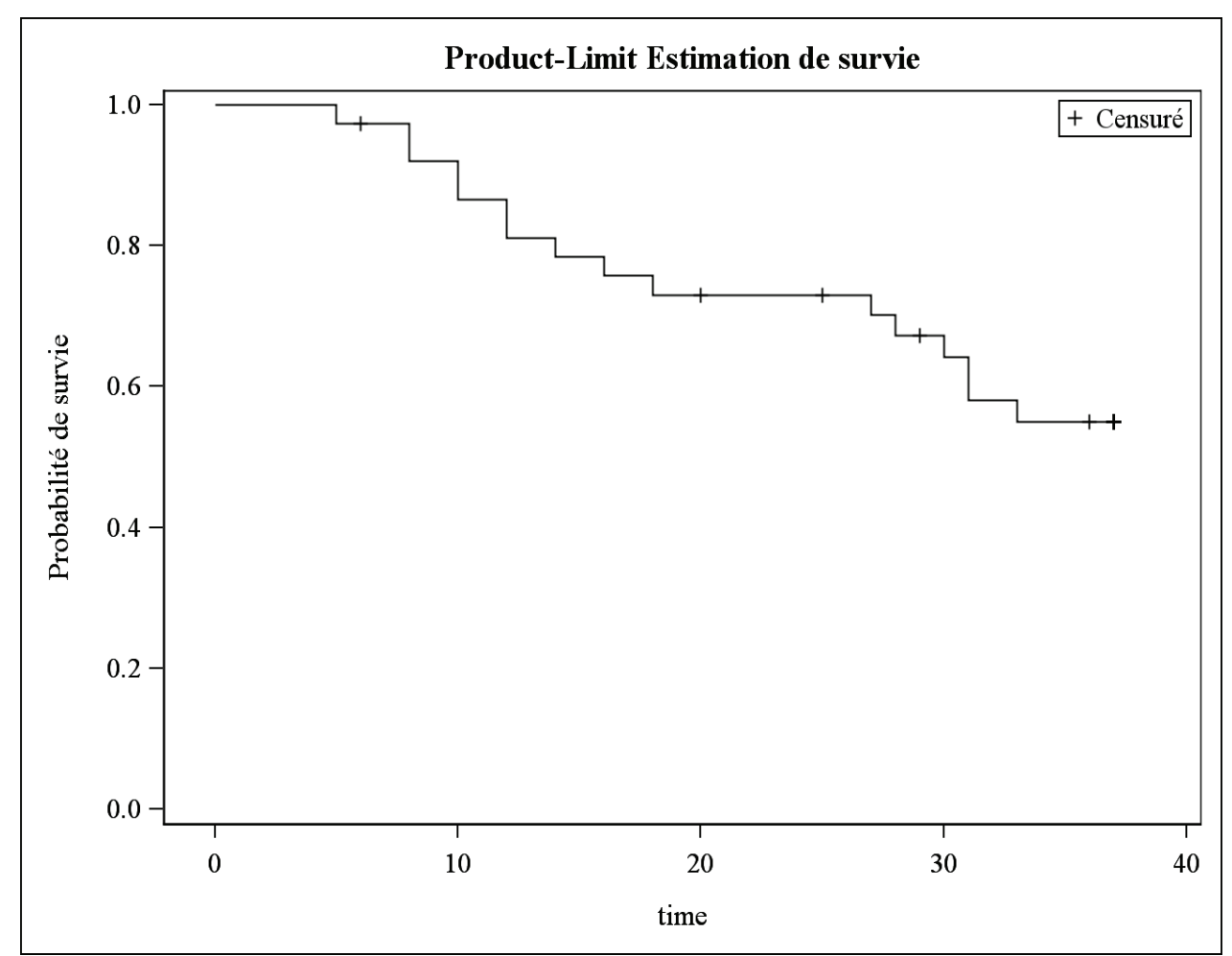

Figure 34 : Courbe de survie globale à 3 ans. 


\section{- Taux de survie et âge moyen :}

Le taux de survie globale à 3 ans était de :

- $71,3 \%$, IC $95 \%[44,0 \% ; 87,0 \%]$ pour les sujets de moins de 64 ans.

- $\quad 42,2 \%$ IC $95 \%[20,4 \% ; 62,6 \%]$ pour les patients de 64 ans et plus.

Néanmoins, il n'était pas retrouvé de corrélation significative entre l'âge moyen des patients et le taux de survie globale ( $p=0,16$, d'après le test du Log-rang).

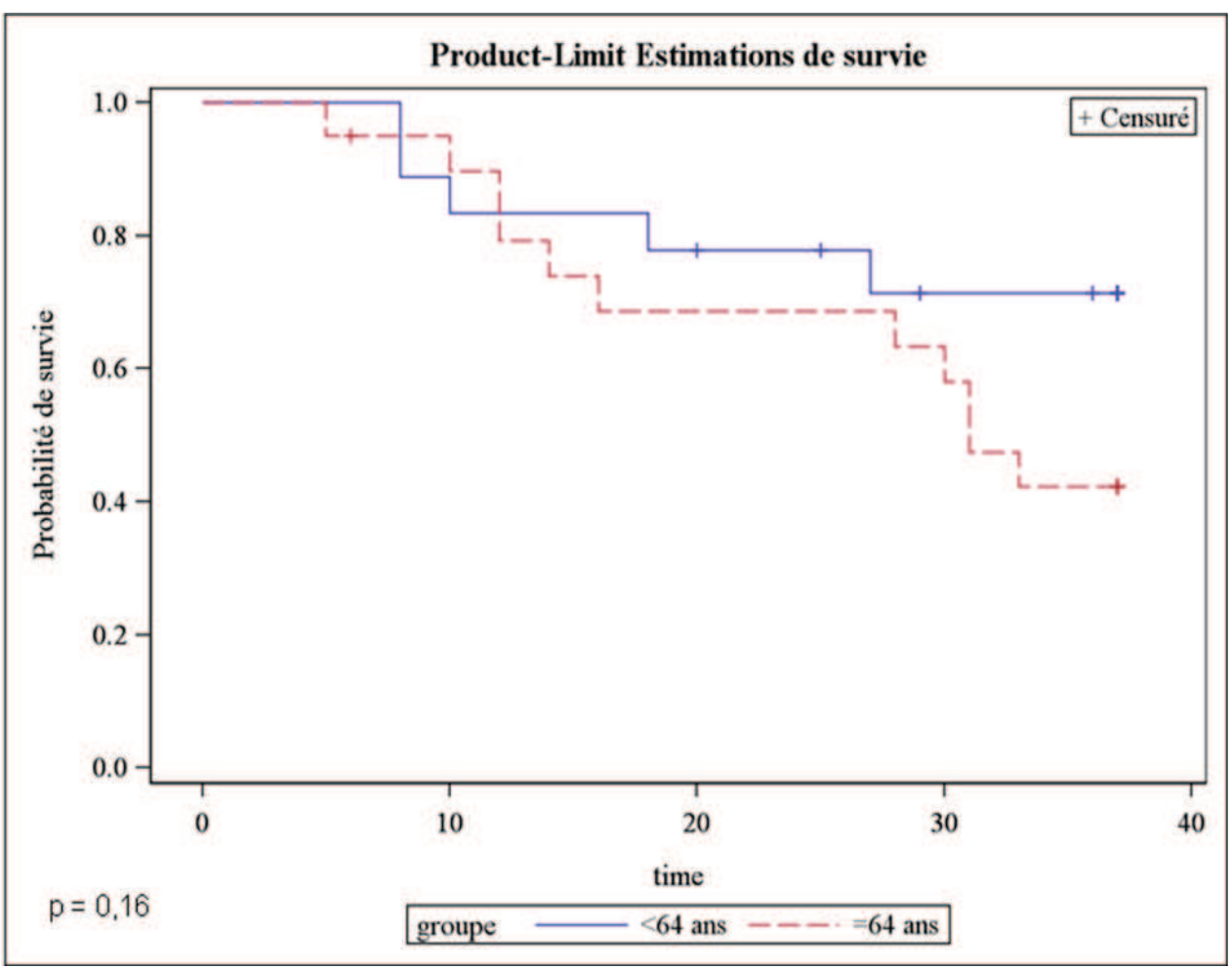

Figure 35 :

Courbe de survie globale à 3 ans en fonction de l'âge ( $\geq$ ou < à 64 ans). 
- Taux de survie et classification TNM :

Le taux de survie à 3 ans était de :

- $79 \%$, IC $95 \%[47,9 \% ; 92,7 \%]$ pour les groupes « $\mathrm{T}_{1}$ et $\mathrm{T}_{2}$ »

- $38,2 \%$ IC 95\% [18\%; 58,3\%] pour les groupes « $\mathrm{T}_{3}$ et $\mathrm{T}_{4} »$.

Cette corrélation était significative ( $p=0,0085$ d'après le test du Log-rang), le taux de survie à 3 ans étant significativement plus important chez les sujets dont la taille de la tumeur initiale était classée « $T_{1}$ ou $T_{2}$.

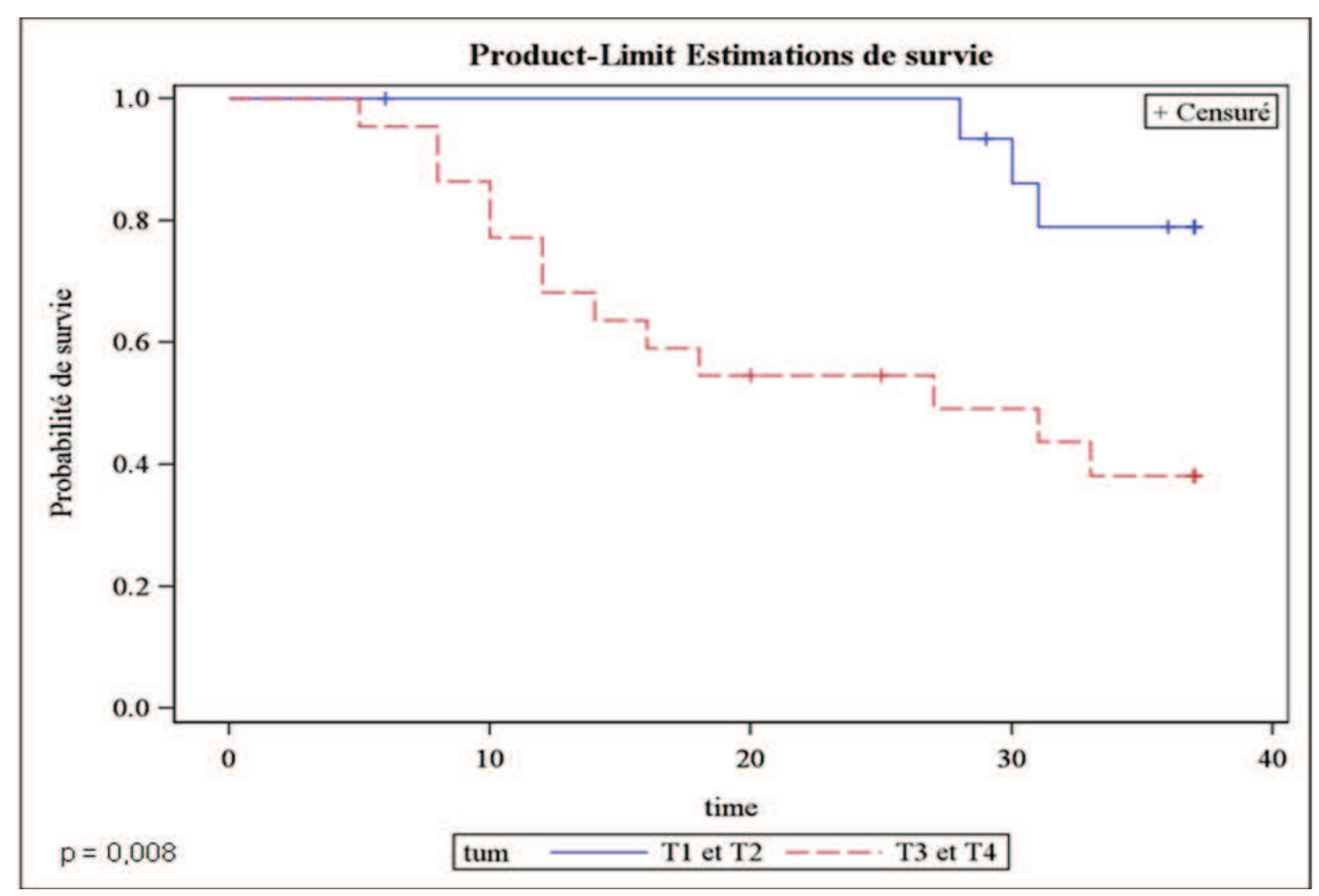

Figure 36 :

Courbe de survie globale à 3 ans en fonction du stade TNM. 
De même, un sujet dont le stade de la tumeur initiale était « $T_{3}$ ou $T_{4}$ » avait 4,6 fois plus de risque de décéder dans les suites de sa pathologie, dans les 3 ans, par rapport à un sujet dont la tumeur était « T1 ou T2 » (selon le modèle de Cox).

Tableau 9 : Modèle de Cox, sans facteur de confusion.

\begin{tabular}{|c|c|c|c|c|c|c|c|c|c|}
\hline \multicolumn{10}{|c|}{ Estimations par l'analyse du maximum de vraisemblance } \\
\hline Paramètre & DDL & $\begin{array}{l}\text { Valeur estimée } \\
\text { des paramètres }\end{array}$ & $\begin{array}{r}\text { Erreur } \\
\text { type }\end{array}$ & Khi-2 & $\operatorname{Pr}>\mathrm{Khi}-2$ & $\begin{array}{r}\text { Rapport } \\
\text { de risque }\end{array}$ & $\begin{array}{l}\text { Inter } \\
\text { confian } \\
\text { du ra } \\
\text { ris }\end{array}$ & $\begin{array}{l}\text { e de } \\
\text { à } 95 \% \\
\text { rt de } \\
\text { e }\end{array}$ & Libellé \\
\hline $\begin{array}{l}\text { T3-T4 vs T1- } \\
\text { T2 }\end{array}$ & 1 & 1.53372 & 0.64445 & 5.6639 & 0.0173 & 4.635 & 1.311 & 16.392 & $\begin{array}{l}\text { groupe taille } \\
\text { tumorale }\end{array}$ \\
\hline
\end{tabular}

Après ajustement sur l'âge, les résultats révélaient un « Hazard Ratio » de 4,7 [1,3 ; $16,6]$, soit une valeur quasi identique à celle retrouvée sans ajustement. 


\section{II - Caractéristiques anatomo-pathologiques}

Selon la classification histologique de Barnes, retenue dans l'OMS 2005, les 38 proliférations tumorales initiales se répartissaient de la façon suivante : 3 (8\%) correspondaient à des adénocarcinomes de type «non intestinal » et 35 (92\%) à des adénocarcinomes de type « intestinal ».

Parmi les adénocarcinomes «non intestinaux », un (33\%) était de bas grade et deux (67\%) de haut grade.

Quant aux adénocarcinomes de type "intestinal » (ITAC), $13(37 \%)$ sont de soustype « coliques », $10(28 \%)$ « mucineux », $7(20 \%)$ « mixtes », 3 (8\%) «papillaires » et $2(6 \%)$ « solides ».

\section{ADK de type intestinal $(n=35)$}

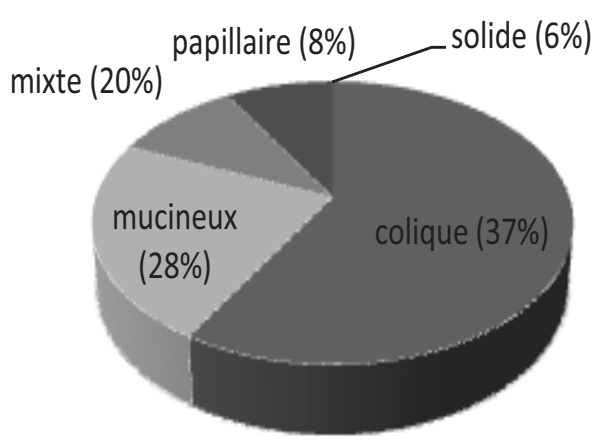

Figure 37 :

Répartition des différentes sous-types d'adénocarcinomes de type intestinal. 
Lorsque les différents paramètres cliniques des patients atteints d'adénocarcinomes nasosinusiens sont corrélés aux divers types histologiques, les résultats suivant sont obtenus :

Aucune différence significative entre les groupes d'adénocarcinomes de type « intestinal » ou non, n'était retrouvée en ce qui concerne l'âge $(p=0,58)$, le sexe $(p=0,22)$ ou la symptomatologie ( $p=0,40$ à 1 en fonction des signes fonctionnels observés).

Par contre, la mise en cause d'une exposition à un facteur de risque (bois ou cuir) était plus fréquemment retrouvée dans le groupe « adénocarcinome de type intestinal » $(85,7 \%)$ que dans le groupe « adénocarcinome de type non intestinal » $(33 \%)$. Néanmoins, cette corrélation n'était pas statistiquement significative $(p=0,08)$.

\section{Tableau 10:}

Test de Fischer, recherche de corrélation entre sous-type histologique et exposition à facteur de risque.

\begin{tabular}{|c|c|c|c|}
\hline \multirow{2}{*}{ Classification Y } & \multicolumn{2}{|c|}{ Classification X } & \\
\cline { 2 - 3 } & $\mathbf{0}$ & $\mathbf{1}$ & \\
\hline NON & 2 & 1 & $3(7,9 \%)$ \\
\hline OUI & 5 & 30 & $35(92,1 \%)$ \\
\hline & 7 & 31 & 38 \\
\hline & $(18,4 \%)$ & $(81,6 \%)$ & \\
\hline $\mathbf{P = 0 , 0 8 1 3 1 8 1 6 0}$ & \multicolumn{2}{|l}{} \\
\hline
\end{tabular}

- Classification X : Exposition (1) ou non (0) à un facteur de risque (bois ou cuir).

- Classification Y : ADK de type « intestinal » (oui) ou " non intestinal » (non). 
La répartition des différentes variantes histologiques en fonction du stade TNM est la suivante :

\section{$\underline{\text { Tableau } 11}$}

\begin{tabular}{|c|c|c|c|c|}
\hline $\begin{array}{l}\text { ADK NON INTESTINAL } \\
\qquad(\mathrm{n}=3)\end{array}$ & \multicolumn{4}{|c|}{ ADK INTESTINAL $(\mathrm{n}=35)$} \\
\hline & $\begin{array}{l}\text { MUCINEUX } \\
(n=10)\end{array}$ & $\begin{array}{l}\text { MIXTE } \\
(n=7)\end{array}$ & $\begin{array}{l}\text { PAPILLAIRE } \\
\quad(n=3)\end{array}$ & $\begin{array}{c}\text { SOLIDE } \\
(n=2)\end{array}$ \\
\hline & 0 & 1 & 0 & 0 \\
\hline & 4 & 2 & 2 & 0 \\
\hline & 1 & 0 & 0 & 0 \\
\hline & 5 & 4 & 1 & 2 \\
\hline
\end{tabular}

- La majorité des tumeurs classées initialement $T_{4} \quad(n=16)$ étaient des adénocarcinomes de type « intestinal, mucineux » $(n=5)$.

- De même, le cas classé $\mathrm{T}_{4} \mathrm{~N}_{2} \mathrm{bM}_{0}$ était également un adénocarcinome de type « intestinal, mucineux ».

- L'étude de la répartition histologique des lésions tumorales ayant récidivé mettait en évidence :

- Pour les récidives locales:

$>$ A la fois au niveau des récidives locales et à distance, l'aspect histologique était identique au primitif.

Sur les 12 cas de récidives locales, 11 (92\%) appartenaient aux adénocarcinomes de type " intestinal », ce qui correspondait à un taux de récidive de $31,4 \%$. 
Le taux de récidive tumorale locale rapportée aux différents sous-types histologiques d'adénocarcinomes « intestinaux » était respectivement de $8 \%$ pour les « coliques », 33\% pour les « papillaires », 40\% pour les « mucineux », $57 \%$ pour les « mixtes » et $100 \%$ pour les « solides ».

La lésion tumorale récidivante localement restante était un adénocarcinome de type "non intestinal », ce qui correspondait à un taux de récidive de $33 \%$ de cette variété histologique.

\section{- Pour les récidives ganglionnaires:}

Les deux cas ayant présenté une métastase ganglionnaire appartenaient aux adénocarcinomes de type «intestinal » (un « colique » et un « mucineux »), ce qui correspondait à un taux de récidive ganglionnaire estimé à $6 \%$.

- Pour les métastases à distance:

5 des 6 cas ayant présenté une métastase à distance appartenaient aux adénocarcinomes de type «intestinal » (3 « mucineux " et 2 « mixtes »), ce qui correspondait à un taux métastatique de $14 \%$.

La lésion tumorale métastatique restante était un adénocarcinome « non intestinal » de haut grade, ce qui correspondait à un taux métastatique de $33 \%$ de cette variété histologique.

Statistiquement, il n'était pas retrouvé de corrélation significative entre le taux de récidive (locale, ganglionnaire ou métastatique) et le sous-type histologique (« intestinal » ou « non intestinal ») de ces adénocarcinomes. 
L'étude du taux de mortalité rapporté aux différents sous-types histologiques, retrouvait des chiffres de $31,42 \%$ pour les ITAC et 33\% pour les adénocarcinomes de type « non intestinal » (résultats non significatifs, $p=1$ ).

II est intéressant de noter que les deux cas d'adénocarcinomes de type "non intestinal » de haut grade sont décédés suite à l'évolution de leur pathologie, alors que l'unique cas d'adénocarcinome de type "non intestinal » de bas grade était considéré comme guéri.

Parmi les adénocarcinomes de type «intestinal », un taux de mortalité nul a été retrouvé pour le sous-type "solide », des taux de 15\%,33\%, 42\% et 50\% ont été notés respectivement pour les sous-types «coliques », "papillaires », " mixtes » et « mucineux». Par ailleurs, le taux de survie globale à 3 ans des sujets ayant présenté une tumeur appartenant aux sous-types « mucineux ou mixte » était de $46,3 \%$, IC 95\% [22,1\%; 67,6\%], alors que les sujets ayant une tumeur « colique, papillaire ou solide » avaient un taux de survie à 3 ans de 61,4\%, IC 95\% [33,2\% ; $80,6 \%$ ]. Néanmoins, aucune corrélation significative n'était retrouvée entre le soustype histologique et le taux de survie globale à 3 ans $(p=0,24$, d'après le test du Log-rang).

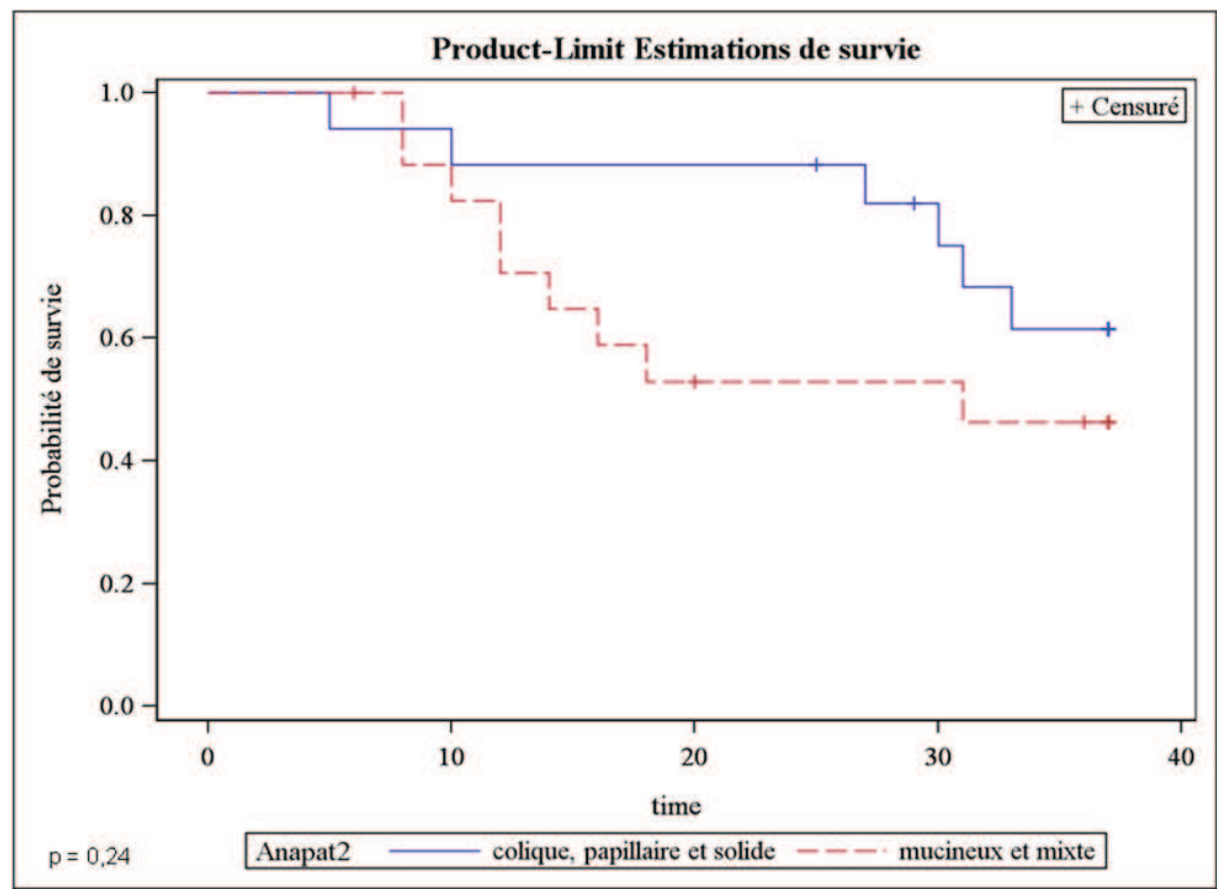

Figure 38:

Courbe de survie globale en fonction des sous-types histologiques d'ITACs. 


\section{III - Caractéristiques immunohistochimiques}

$1-\underline{\mathrm{CDX} 2}$

L'expression de CDX2 par les cellules tumorales a été recherchée sur l'ensemble des 38 cas étudiés (tumeurs primitives). Une positivité était admise dès lors qu'un marquage nucléaire intense et diffus était observé. Les résultats sont répertoriés dans le tableau 12.

Tableau 12: Immunomarquages CDX2

\begin{tabular}{|c|c|c|c|c|c|c|c|c|}
\hline CAS & HISTOLOGIE & CDX2 & CAS & HISTOLOGIE & CDX2 & CAS & HISTOLOGIE & CDX2 \\
\hline 1 & ADK intestinal mucineux & - & 14 & ADK intestinal mucineux & + & 27 & ADK intestinal mucineux & + \\
\hline 2 & ADK intestinal mucineux & + & 15 & ADK intestinal, colique & + & 28 & ADK intestinal, colique & + \\
\hline 3 & ADK intestinal mixte & + & 16 & ADK intestinal, colique & + & 29 & ADK intestinal, colique & + \\
\hline 4 & ADK intestinal mixte & + & 17 & ADK intestinal, colique & + & 30 & ADK intestinal mucineux & + \\
\hline 5 & ADK intestinal mixte & + & 18 & ADK intestinal mucineux & + & 31 & ADK intestinal mucineux & + \\
\hline 6 & ADK intestinal mixte & + & 19 & ADK intestinal, colique & + & 32 & ADK intestinal, colique & + \\
\hline 7 & ADK intestinal mucineux & + & 20 & ADK intestinal mixte & + & 33 & ADK intestinal, papillaire & + \\
\hline 8 & ADK NI, bas grade & - & 21 & ADK intestinal, colique & + & 34 & ADK intestinal, papillaire & + \\
\hline 9 & ADK NI, haut grade & - & 22 & ADK intestinal, solide & + & 35 & ADK intestinal, papillaire & + \\
\hline 10 & ADK intestinal, colique & + & 23 & ADK intestinal mucineux & + & 36 & ADK intestinal mixte & + \\
\hline 11 & ADK intestinal mixte & + & 24 & ADK intestinal, solide & + & 37 & ADK intestinal, colique & + \\
\hline 12 & ADK intestinal, colique & + & 25 & ADK NI, haut grade & - & 38 & ADK intestinal mucineux & - \\
\hline 13 & ADK intestinal, colique & + & 26 & ADK intestinal, colique & + & & & \\
\hline
\end{tabular}

- 94\% $(n=33)$ des adénocarcinomes nasosinusiens de type « intestinal » exprimaient le CDX2.

- Parmi les 2 adénocarcinomes nasosinusiens de type « intestinal » n'exprimant pas le CDX2, l'un était le siège d'artéfacts, liés à une décalcification préalable du bloc de paraffine.

- 100\% $(n=3)$ des adénocarcinomes nasosinusiens de type «non intestinal » restaient négatifs pour cet anticorps. 


\section{2 - $\underline{\text { Cytokératines } 7 \text { et } 20}$}

L'expression des cytokératines 7 et 20 par les cellules tumorales a été recherchée sur l'ensemble des 38 cas étudiés (tumeurs primitives). Une positivité était admise dès lors qu'un marquage cytoplasmique à renforcement membranaire était observé. Les résultats sont répertoriés dans les tableau 13.

Tableau 13: Immunomarquages CK20 et CK7.

\begin{tabular}{|c|c|c|c|c|c|c|c|c|}
\hline CAS & HISTOLOGIE & $\begin{array}{c}\text { CK20 } \\
\text { CK7 }\end{array}$ & CAS & HISTOLOGIE & $\begin{array}{c}\text { CK20 } \\
\text { CK7 }\end{array}$ & CAS & HISTOLOGIE & $\begin{array}{c}\text { CK20 } \\
\text { CK7 }\end{array}$ \\
\hline 1 & ADK intestinal mucineux & - & 14 & ADK intestinal mucineux & $\begin{array}{l}+ \\
+\end{array}$ & 27 & ADK intestinal mucineux & $\begin{array}{l}+ \\
-\end{array}$ \\
\hline 2 & ADK intestinal mucineux & $\begin{array}{l}+ \\
+\end{array}$ & 15 & ADK intestinal, colique & $\begin{array}{l}+ \\
-\end{array}$ & 28 & ADK intestinal, colique & $\begin{array}{l}+ \\
-\end{array}$ \\
\hline 3 & ADK intestinal mixte & $\begin{array}{l}+ \\
+\end{array}$ & 16 & ADK intestinal, colique & $\begin{array}{l}+ \\
+\end{array}$ & 29 & ADK intestinal, colique & $\begin{array}{l}+ \\
+\end{array}$ \\
\hline 4 & ADK intestinal mixte & $\begin{array}{l}+ \\
-\end{array}$ & 17 & ADK intestinal, colique & $\begin{array}{l}+ \\
-\end{array}$ & 30 & ADK intestinal mucineux & $\begin{array}{l}+ \\
-\end{array}$ \\
\hline 5 & ADK intestinal mixte & $\begin{array}{l}+ \\
+\end{array}$ & 18 & ADK intestinal mucineux & $\begin{array}{l}+ \\
-\end{array}$ & 31 & ADK intestinal mucineux & $\begin{array}{l}+ \\
-\end{array}$ \\
\hline 6 & ADK intestinal mixte & $\begin{array}{l}+ \\
+\end{array}$ & 19 & ADK intestinal, colique & $\begin{array}{l}+ \\
+\end{array}$ & 32 & ADK intestinal, colique & $\begin{array}{l}+ \\
+\end{array}$ \\
\hline 7 & ADK intestinal mucineux & - & 20 & ADK intestinal mixte & $\begin{array}{l}+ \\
+\end{array}$ & 33 & ADK intestinal, papillaire & $\begin{array}{l}+ \\
+\end{array}$ \\
\hline 8 & ADK NI, bas grade & + & 21 & ADK intestinal, colique & $\begin{array}{l}+ \\
-\end{array}$ & 34 & ADK intestinal, papillaire & $\begin{array}{l}+ \\
+\end{array}$ \\
\hline 9 & ADK NI, haut grade & - & 22 & ADK intestinal, solide & $\begin{array}{l}+ \\
-\end{array}$ & 35 & ADK intestinal, papillaire & $\begin{array}{l}+ \\
-\end{array}$ \\
\hline 10 & ADK intestinal, colique & $\begin{array}{l}+ \\
-\end{array}$ & 23 & ADK intestinal mucineux & $\begin{array}{l}+ \\
+\end{array}$ & 36 & ADK intestinal mixte & $\begin{array}{l}+ \\
-\end{array}$ \\
\hline 11 & ADK intestinal mixte & $\begin{array}{l}+ \\
+ \\
\end{array}$ & 24 & ADK intestinal, solide & $\begin{array}{l}+ \\
-\end{array}$ & 37 & ADK intestinal, colique & $\begin{array}{l}+ \\
-\end{array}$ \\
\hline 12 & ADK intestinal, colique & $\begin{array}{l}+ \\
- \\
\end{array}$ & 25 & ADK NI, haut grade & $\begin{array}{l}- \\
+\end{array}$ & 38 & ADK intestinal mucineux & $\begin{array}{l}+ \\
- \\
\end{array}$ \\
\hline 13 & ADK intestinal, colique & $\begin{array}{l}+ \\
-\end{array}$ & 26 & ADK intestinal, colique & $\begin{array}{l}+ \\
+\end{array}$ & & & \\
\hline
\end{tabular}

ADK : adénocarcinome ; NI : non intestinal ; + :immunomarquage cytoplasmqiue positif ; - : absence d'immunomarquage.

- 94\% ( $n=33)$ des adénocarcinomes nasosinusiens de type « intestinal » exprimaient la CK 20 et 100\% ( $n=3)$ des adénocarcinomes nasosinusiens de type «non intestinal » restaient négatifs pour cet anticorps.

- 43\% $(n=15)$ des adénocarcinomes de type «intestinal » et $66 \% \quad(n=2)$ des adénocarcinomes nasosinusiens de type « non intestinal » exprimaient la CK7. 


\section{$3-\underline{E G F R}$}

L'évaluation quantitative des immunomarquages par l'anticorps EGFR a été effectuée sur l'ensemble des prélèvements intéressant les tumeurs primitives des 38 patients. Elle était considérée comme (figure 39) :

- Négative $(0+)$ : absence de marquage par les cellules tumorales.

- Faiblement positive $(1+)$ : marquage faible et focal, membranaire.

- Modérément positive (2+) : marquage membranaire d’intensité modérée.

- Très positive $\left(3^{+}\right)$: marquage membranaire fort et circonférentiel.

Le pourcentage de cellules tumorales exprimant cette protéine a également été quantifié. Les résultats sont répertoriés dans le tableau 14.

Tableau 14 : Immunomarquages EGFR

\begin{tabular}{|c|c|c|c|c|c|c|c|c|}
\hline CAS & HISTOLOGIE & EGFR & CAS & HISTOLOGIE & EGFR & CAS & HISTOLOGIE & EGFR \\
\hline 1 & ADK intestinal mucineux & $3+90 \%$ & 14 & ADK intestinal mucineux & $1+5 \%$ & 27 & ADK intestinal mucineux & $0+$ \\
\hline 2 & ADK intestinal mucineux & $2+30 \%$ & 15 & ADK intestinal, colique & $1+20 \%$ & 28 & ADK intestinal, colique & $1+70 \%$ \\
\hline 3 & ADK intestinal mixte & $2+40 \%$ & 16 & ADK intestinal, colique & $1+1 \%$ & 29 & ADK intestinal, colique & $1+<1 \%$ \\
\hline 4 & ADK intestinal mixte & $1+10 \%$ & 17 & ADK intestinal, colique & $3+20 \%$ & 30 & ADK intestinal mucineux & $1+5 \%$ \\
\hline 5 & ADK intestinal mixte & $1+5 \%$ & 18 & ADK intestinal mucineux & $1+1 \%$ & 31 & ADK intestinal mucineux & $0+$ \\
\hline 6 & ADK intestinal mixte & $2+40 \%$ & 19 & ADK intestinal, colique & $2+20 \%$ & 32 & ADK intestinal, colique & 2 à $3+30 \%$ \\
\hline 7 & ADK intestinal mucineux & $0+$ & 20 & ADK intestinal mixte & $3+10 \%$ & 33 & ADK intestinal, papillaire & $1+<1 \%$ \\
\hline 8 & ADK NI, bas grade & $1+20 \%$ & 21 & ADK intestinal, colique & $1+5 \%$ & 34 & ADK intestinal, papillaire & $0+$ \\
\hline 9 & ADK NI, haut grade & 1 à $2+60 \%$ & 22 & ADK intestinal, solide & $0+$ & 35 & ADK intestinal, papillaire & $1+1 \%$ \\
\hline 10 & ADK intestinal, colique & $1+1 \%$ & 23 & ADK intestinal mucineux & $2+40 \%$ & 36 & ADK intestinal mixte & $1+2 \%$ \\
\hline 11 & ADK intestinal mixte & 0 à $1+5 \%$ & 24 & ADK intestinal, solide & $1+1 \%$ & 37 & ADK intestinal, colique & 1 à $2+80 \%$ \\
\hline 12 & ADK intestinal, colique & $1+30 \%$ & 25 & ADK NI, haut grade & $1+1 \%$ & 38 & ADK intestinal mucineux & $0+$ \\
\hline 13 & ADK intestinal, colique & $1+1 \%$ & 26 & ADK intestinal, colique & $1+1 \%$ & & & \\
\hline
\end{tabular}

ADK: adénocarcinome; $\mathrm{NI}$ : non intestinal; + :immunomarquage membranaire positif ; - : absence d'immunomarquage.

- $84 \%$ des cas d'ITAC et $100 \%$ des cas d'adénocarcinomes nasosinusiens de type «non intestinal » exprimaient la protéine EGFR (marquage hétérogène et très variable, allant de $1+1 \%$ à $3+90 \%)$. 
- II n'a pas été retrouvé de différence significative en ce qui concerne l'expression d'EGFR par les cellules tumorales et les différents sous-types histologiques de ces tumeurs. De même, il n'a pas été retrouvée de corrélation significative entre l'expression de la protéine EGFR et l'exposition à un facteur de risque $(p=0,3)$.

- Sur les 38 cas, $6(16 \%)$, tous de type « intestinal » (4 « mucineux », un « solide » et un "papillaire »), ne montraient aucune expression de la protéine EGFR par les cellules tumorales. A noter que l'un d'entre eux (cas $n^{\circ} 38$ ), était le siège d'artéfacts liés à une décalcification préalable du bloc paraffiné.

- Une expression faible (1+) était mise en évidence dans la majorité des cas $(n=21$, soit $55 \%$ ), avec un pourcentage de cellules marquées variant de $<1 \%$ à $70 \%$, selon les cas. Dix-neuf $(90 \%)$ étaient des adénocarcinomes de type "intestinal " (9 " coliques », 4 « mixtes », 3 « mucineux », 2 « papillaires » et un « solide ») et deux $(10 \%)$ étaient des adénocarcinomes de type "non intestinal » (un de «bas grade » et un de « haut grade »).

- Une expression modérée (2+) était mise en évidence dans 7 cas (18\%), avec un pourcentage de cellules marquées variant de 20 à $80 \%$ selon les cas. Six d'entre eux (86\%) étaient de type « intestinal » (2 « coliques », 2 « mucineux », 2 « mixtes »). Le cas restant était un adénocarcinome de type « non intestinal de haut grade ».

- Une expression intense (3+) était retrouvée pour 4 cas (10\%), tous de type " intestinal» (2 «coliques», 1 «mucineux» et 1 «mixte»). Pour un cas, l'expression était diffuse (90\%), pour les deux autres, elle était focale (10 à $30 \%$ de cellules positives).

- II apparait important de souligner que 100\% des cas d'adénocarcinomes de type « intestinal colique ou mixte » présentaient une positivité de cet immunomarquage. 


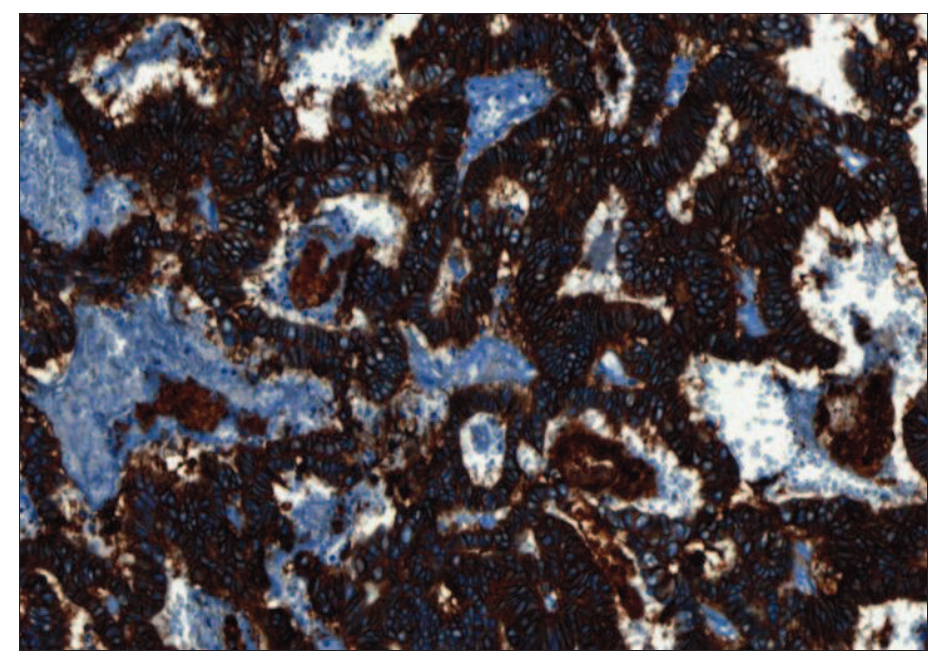

Figure $39_{\mathrm{A}}$ :

Immunomarquage

EGFR 3+

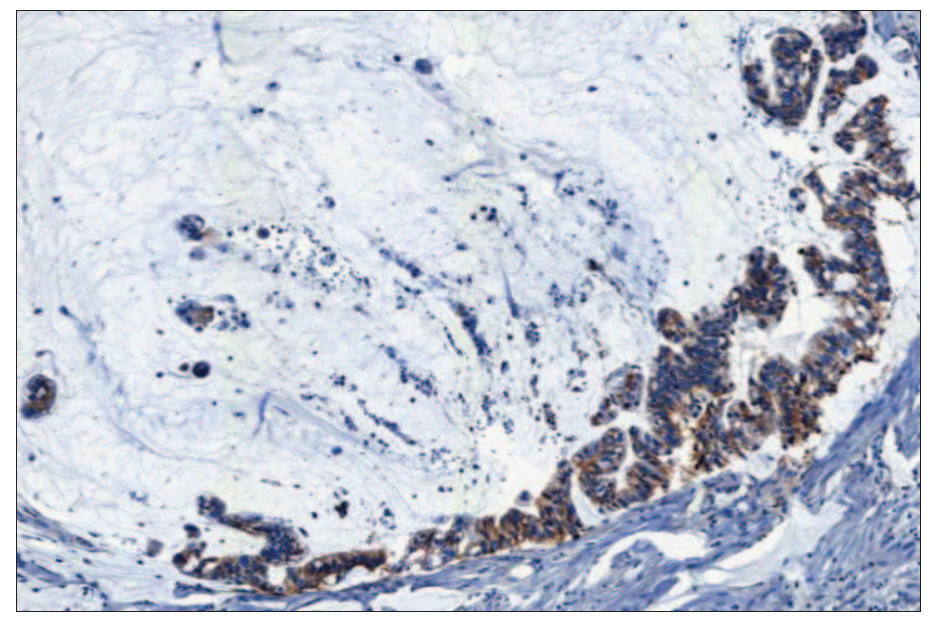

Figure $39_{B}$ : Immunomarquage EGFR 2+

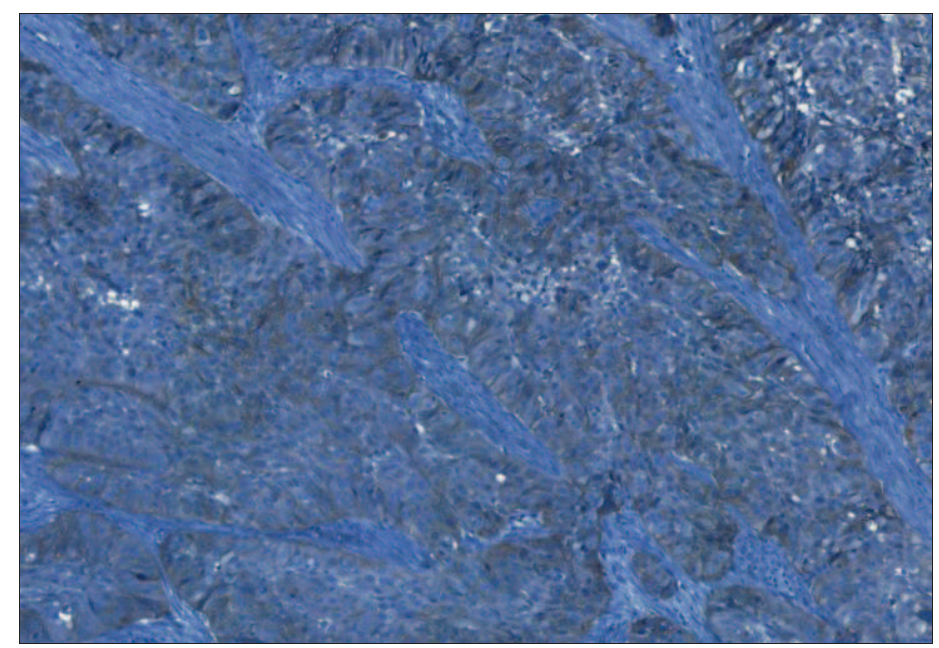

Figure $39 \mathrm{c}$ : Immunomarquage EGFR 1+ 


\section{IV - Répartition des mutations EGFR/KRAS}

Sur les 38 cas examinés (tableau 15):

- Aucune mutation du gène EGFR n'a été retrouvée.

- 33 cas $(87 \%)$ ne présentaient pas de mutation ponctuelle du gène KRAS.

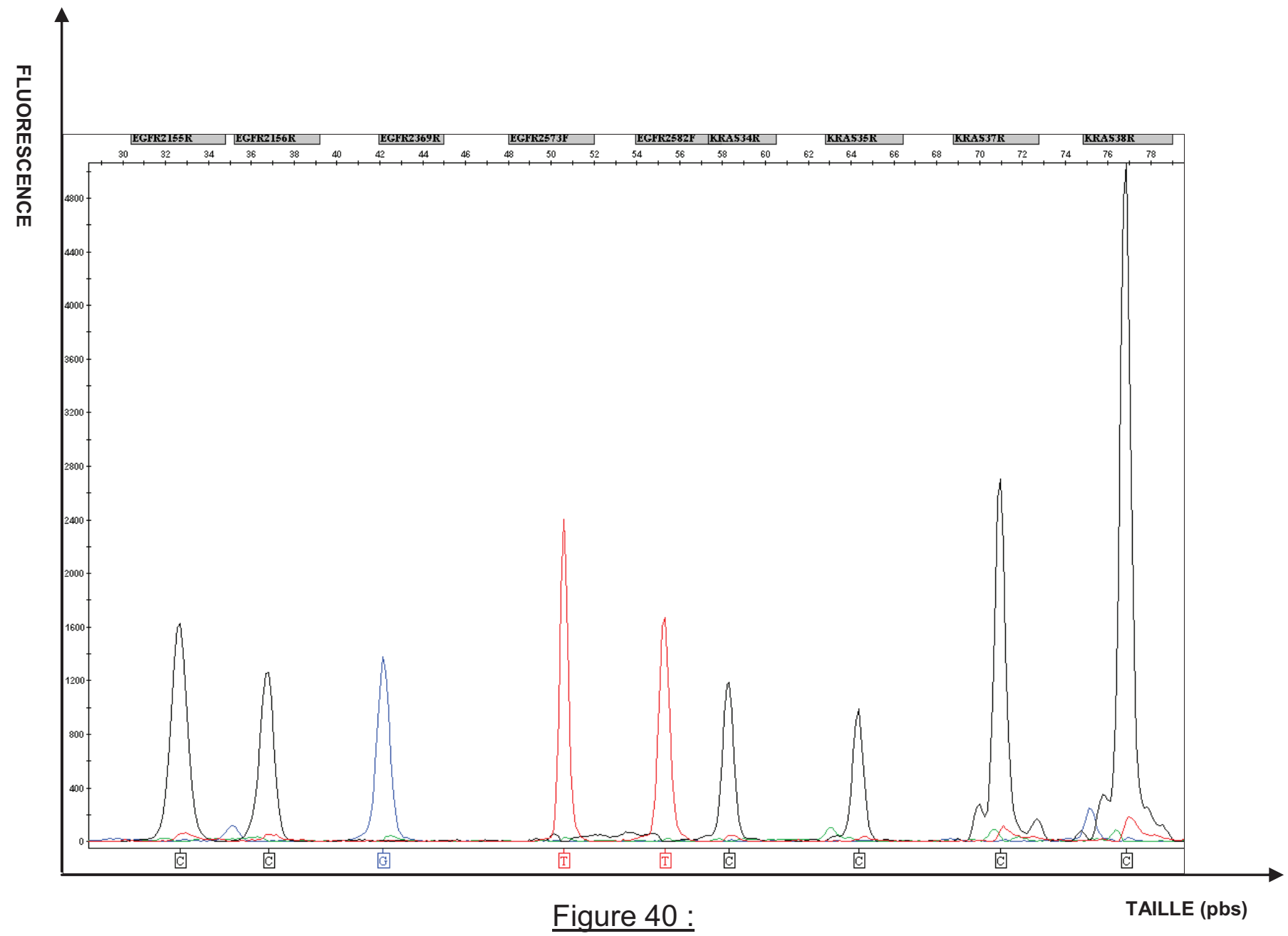

Profil SNaPshot@ EGFR / KRAS « sauvage » (wt), retrouvé chez 33 patients. 
Tableau 15 / TABLEAU BIOMOL

\begin{tabular}{|l|l|l|l|}
\hline & & & \\
\hline & & & \\
\hline
\end{tabular}


- 5 patients (13\%) présentaient une mutation ponctuelle du gène KRAS :

\section{Cas $n^{\circ} 2:$ c.35G>A; p.G12D.}

Cas $\mathrm{n}^{\circ} 3: \mathbf{c . 3 4 G > T} ; \mathbf{p . G 1 2 C}$ (retrouvée à la fois sur la tumeur primitive et la récidive locale).

Cas $n^{\circ} 4:$ c.38G>A; p.G13D (retrouvée au niveau de la tumeur primitive) et c.35G>A; p.G12D (au niveau de la récidive locale).

Cas $n^{\circ} 11$ : c. 35G>A ; p.G12D (retrouvée sur la métastase viscérale à distance et sur les pièces de récidives locales mais pas sur la tumeur primitive).

\section{Cas $n^{\circ} 17:$ c.35G>C ; p.G12A.}

II apparait important de souligner que :

- $80 \%$ des profils mutés intéressent le nucléotide c.35 (codon 12 de l'exon 2) du gène KRAS.

- $60 \%$ des mutations sont de type : c.35G>A, p.G12D. 
Figure 41 : Profils SNaPshot des 5 cas présentant une mutation du gène KRAS
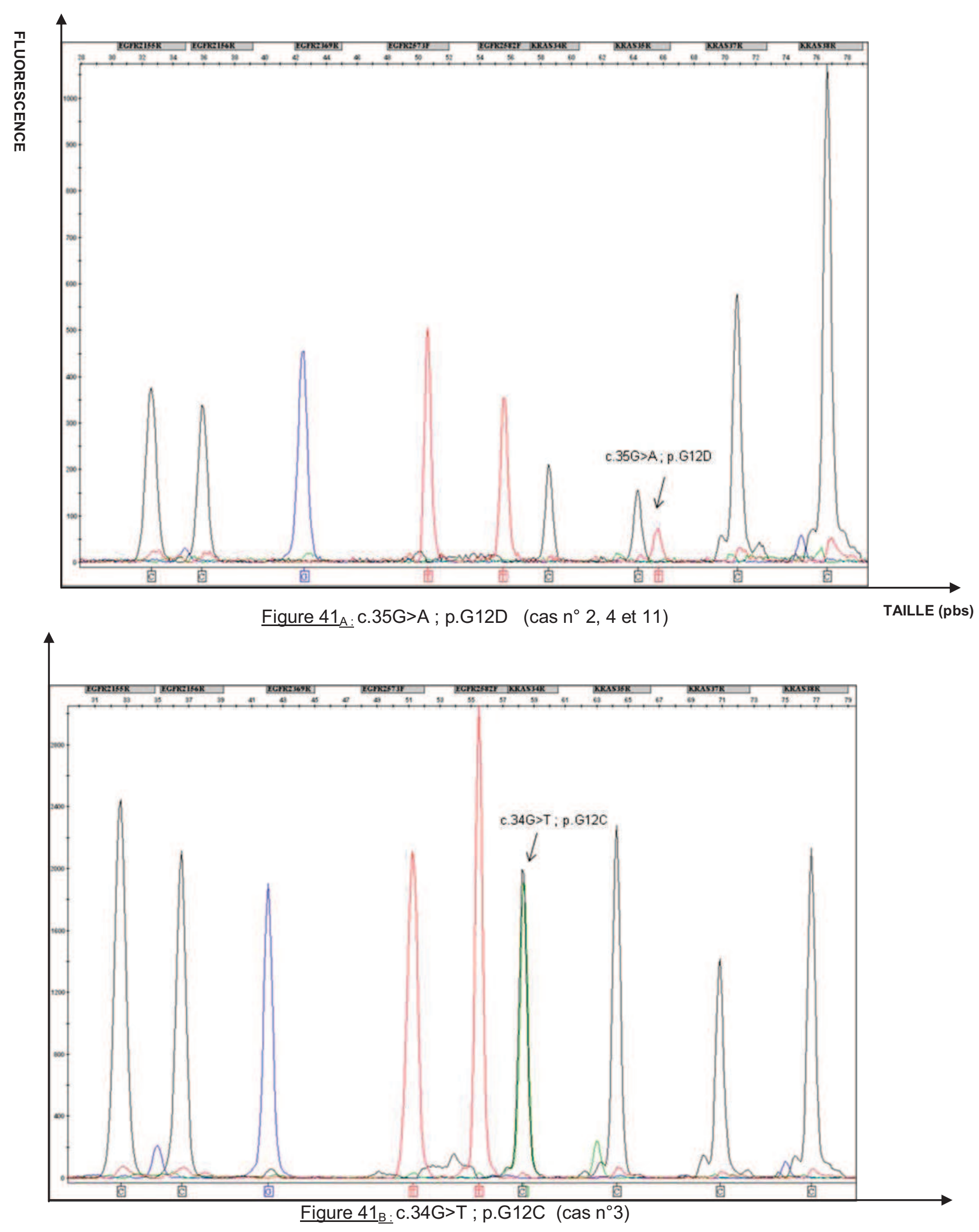

127 

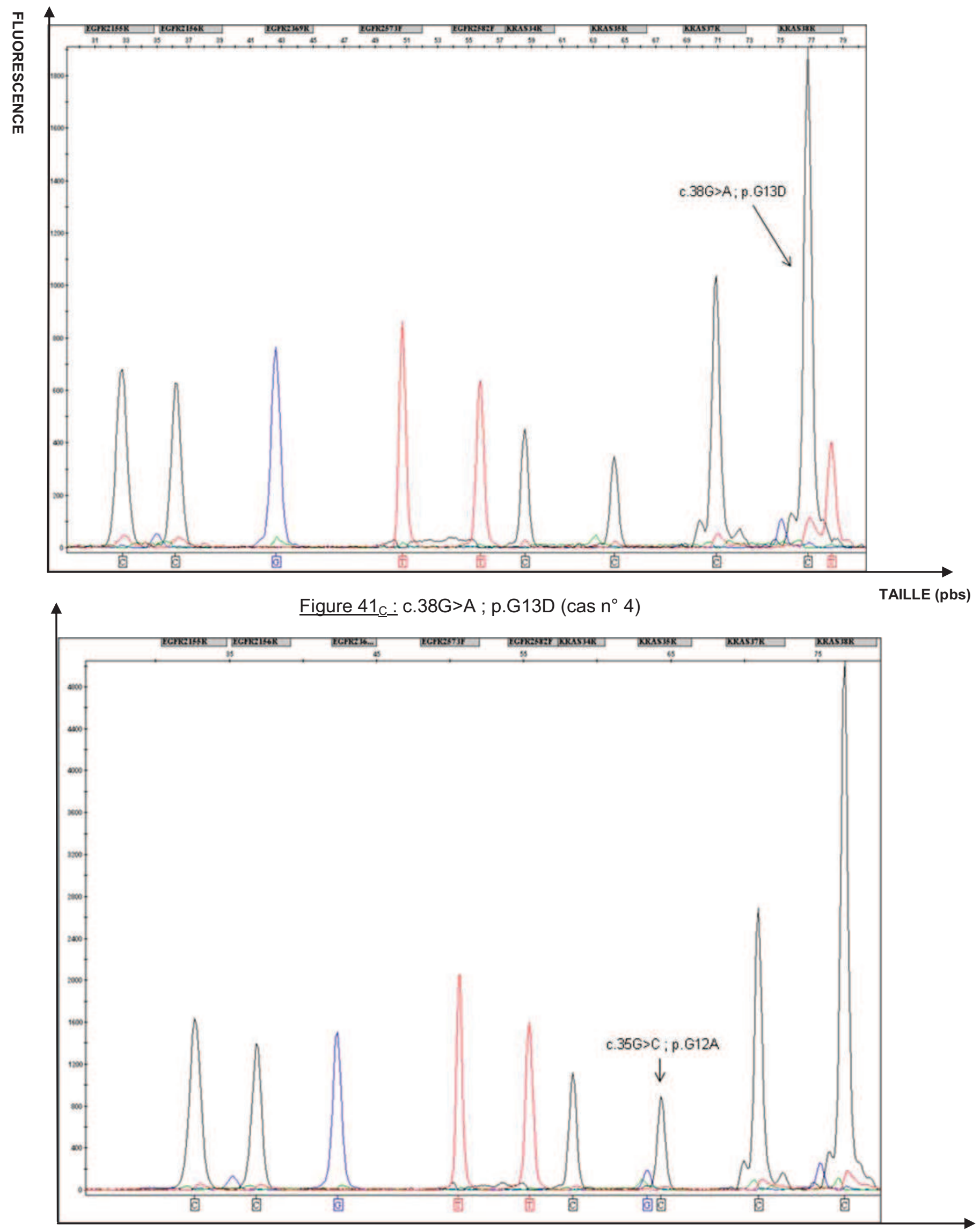

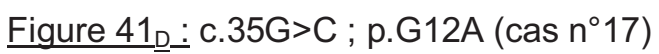




\section{V - Recherche de corrélation entre le profil mutationnel KRAS et les données anatomo-cliniques}

1 - Corrélation entre le statut mutationnel et les données cliniques

Lorsque les différents paramètres cliniques sont corrélés au profil mutationnel, les résultats suivant sont obtenus :

- L'ensemble des profils mutationnels sont observés chez des sujets de sexe masculin, âgés de 46 à 59 ans $(p=1)$.

- Tous les patients présentaient un signe fonctionnel commun : l'obstruction nasale $(p=0,15)$.

- Quatre patients sur $5(80 \%)$ avaient été exposés au bois $(p=1)$, le délai d'exposition variant de 5 à 47 ans.

- Pour 3 cas $(60 \%)$, une récidive locale ou métastatique était observée $(p=0,65)$.

- Un seul cas (20\%) est décédé des suites de sa pathologie tumorale $(p=1)$.

Aucune corrélation significative n'était retrouvée pour l'ensemble de ces variables, probablement en raison d'un effectif trop faible au sein de chaque échantillon. 
Lorsque ces différents profils mutationnels sont comparés à la classification TNM, il est noté :

- 1 cas était initialement classé $\mathrm{T}_{1} \mathrm{~N}_{0} \mathrm{M}_{0}$ (taux de mutation évalué à $100 \%$ ).

- 3 cas étaient initialement classés $\mathrm{T}_{2} \mathrm{~N}_{0} \mathrm{M}_{0}$ (taux de mutation évalué à $20 \%$ ).

- 1 cas était initialement classé $\mathrm{T}_{3} \mathrm{~N}_{0} \mathrm{M}_{0}$ (taux de mutation évalué à $17 \%$ ).

- Aucun cas initialement classé $\mathrm{T}_{4} \mathrm{~N}_{0} \mathrm{M}_{0}$ ne présentait de mutation.

2 - Corrélation entre le statut mutationnel et les données anatomo-pathologiques

L'intégralité des patients chez qui une mutation du gène KRAS a été mise en évidence présentait un adénocarcinome nasosinusien de type « intestinal », dont :

3 de type « mixte », soit un taux mutationnel estimé à $42 \%$.

$>1$ de type « mucineux », soit un taux mutationnel estimé à $10 \%$.

1 de type « colique », soit un taux mutationnel estimé à $8 \%$.

Par ailleurs, aucun cas d'adénocarcinome nasosinusien de type « intestinal solide ou papillaire » et de type "non intestinal de bas ou haut grade " n'a présenté de mutation du gène KRAS. De plus, il n'a pas été retrouvée de corrélation significative entre le sous type histologique et le profil mutationnel des patients $(p=1)$. 


\section{VI - Profil mutationnel tumeurs primitives / récidives locales / métastases}

Sur les 5 cas présentant une mutation du gène KRAS, 3 couples primitifs/récidives ont été étudiés. Les résultats suivant étaient observés :

$>$ Pour l'un d'entre eux, la mutation retrouvée était identique au niveau du primitif et de la récidive locale.

$>$ Un cas présentait une mutation différente au niveau du primitif et de la récidive locale, l'une intéressant le codon 12 et l'autre le codon 13 du gène KRAS.

Chez un patient, aucune mutation n'était retrouvée sur la lésion primitive, alors que les récidives locale et à distance étaient le siège d'une mutation, identique pour les différents prélèvements, du gène KRAS.

Soit un taux de discordance mutationnel du gène KRAS estimé à $66 \%$ (2 cas discordants / 3 cas mutés). 


\section{DISCUSSION}

\section{1 - Analyse des caractéristiques épidémiologiques, cliniques et}

\section{thérapeutiques}

Cette analyse, rétrospective, intéressait 38 patients atteints d'adénocarcinomes nasosinusiens, diagnostiqués dans les services d'ORL et d'Anatomie et de Cytologie Pathologiques du CHU de Rouen, entre 1986 et 2010.

Concernant les données épidémiologiques [9-21], les résultats retrouvés dans notre étude étaient superposables à ceux de la littérature (cf tableau 16). En effet, cette pathologie rare (en moyenne 1,5 cas/an recensés dans notre $\mathrm{CHU}$ ces 24 dernières années), a été essentiellement retrouvée chez des sujets de sexe masculin (92\% d'hommes atteints dans notre série, avec un sexe ratio de 11,7H/1F), âgés d'une soixantaine d'années (moyenne d'âge de 64 ans pour notre étude).

Nos résultats, comme ceux de la littérature, montraient une importante corrélation entre la survenue de ces lésions et une exposition aux poussières organiques de bois durs ou de cuirs (retrouvée dans environ $82 \%$ des cas de notre série) [120].

La durée d'exposition, variable selon les séries, est généralement longue, comprise entre 22 et 31,5 ans [9]. Dans notre étude, elle était en moyenne de 27 ans. Cependant, quelques publications rapportent des cas survenant après un délai d'exposition court, mais dont le niveau d'exposition était plus élevé. Cela était le cas pour 6 patients de notre étude, où une durée d'exposition inférieure ou égale à 10 ans était notée. 
De rares cas sporadiques ont été rapportés, notamment chez les sujets de sexe féminin. Dans notre étude, 6 patients (4 hommes et 2 femmes) n'ont pas été exposés à un facteur de risque.

\section{Tableau 16:}

Principales séries rétrospectives d'adénocarcinomes nasosinusiens rapportées depuis 10 ans.

\begin{tabular}{|c|c|c|c|c|c|c|c|c|c|}
\hline \multirow{2}{*}{$\begin{array}{c}\text { AUTEURS } \\
\text { ANNEE }\end{array}$} & \multirow{2}{*}{$\begin{array}{c}N \\
(H / F)\end{array}$} & \multirow{2}{*}{$\begin{array}{c}\text { AGE } \\
\text { (ANNEES) }\end{array}$} & \multirow{2}{*}{$\begin{array}{c}\text { EXPOSITION AU } \\
\text { BOIS (\%) }\end{array}$} & \multicolumn{4}{|c|}{ GRADE T (\%) } & \multirow{2}{*}{$\begin{array}{c}\text { SURVIE A } 5 \\
\text { ANS (\%) }\end{array}$} & \multirow{2}{*}{$\begin{array}{c}\text { RECIDIVE } \\
(\%)\end{array}$} \\
\hline & & & & $T_{1}$ & $T_{2}$ & $T_{3}$ & $T_{4}$ & & \\
\hline $\begin{array}{l}\text { Breheret, } \\
2011 \text { [9] }\end{array}$ & $41 / 1$ & 61 & 85 & 12 & 31 & 9 & 48 & 44,2 & 46 \\
\hline $\begin{array}{c}\text { De Gabory, } \\
2010 \text { [10] }\end{array}$ & $87 / 8$ & 64 & 73 & 2 & 22 & 37 & 39 & 78 & 31 \\
\hline $\begin{array}{l}\text { Choussy, } \\
2008 \text { [11] }\end{array}$ & 418 & 63 & 85 & 3 & 32 & 23 & 42 & 77 & 51 \\
\hline $\begin{array}{l}\text { Fontana, } \\
2008[12]\end{array}$ & 50 & 63 & 92 & - & - & - & - & - & - \\
\hline $\begin{array}{l}\text { Percodani, } \\
2005 \text { [13] }\end{array}$ & $40 / 6$ & 61 & 69,5 & 6,5 & 26 & 26 & 41,5 & 62 & - \\
\hline $\begin{array}{c}\text { Lietin, } 2005 \\
{[14]}\end{array}$ & $59 / 1$ & 62 & 75 & 13 & 10 & 22 & 55 & 47 & - \\
\hline $\begin{array}{c}\text { Jegoux, } 2004 \\
{[15]}\end{array}$ & $78 / 2$ & 63 & 65 & 5 & 23 & 31 & 41 & 63 & 44 \\
\hline $\begin{array}{c}\text { Michel, } 2003 \\
{[16]}\end{array}$ & 10 & - & - & - & - & - & - & 53 & 60 \\
\hline $\begin{array}{l}\text { Guillotte, } \\
2003 \text { [17] }\end{array}$ & 17 & - & - & 0 & 12 & 0 & 88 & 41 & 58 \\
\hline $\begin{array}{c}\text { Claus, } 2002 \\
{[18]}\end{array}$ & 47 & - & 81 & 4 & 36 & 23 & 36 & 60 & - \\
\hline $\begin{array}{c}\text { Dulguerov, } \\
2001 \text { [19] }\end{array}$ & 25 & - & - & - & - & - & - & 63 & - \\
\hline $\begin{array}{l}\text { Choussy, } \\
2001 \text { [20] }\end{array}$ & 19 & 57 & 75 & 0 & 37 & 50 & 13 & 69 & - \\
\hline $\begin{array}{c}\text { Stoll, } 2001 \\
{[21]}\end{array}$ & $71 / 5$ & 61 & - & 3 & 18 & 58 & 20 & 80 & 23 \\
\hline $\begin{array}{c}\text { Notre étude*, } \\
2012\end{array}$ & $35 / 3$ & 64 & 81,6 & 2,6 & 39,5 & 15,8 & 42 & - & 33,6 \\
\hline
\end{tabular}

*En sachant que les articles du Docteur Choussy [11][20] comportent en partie les mêmes patients que ceux de notre série. 
Les symptômes les plus précoces diffèrent peu des signes rhinologiques bénins. Ces signes sont présents dans la majorité des cas et peuvent régresser temporairement sous traitement antibiotique, rassurant faussement le praticien et le patient [24-28]. Ce ne sont que les symptômes les plus alarmants (oculaires, neurologiques, ou l'apparition d'une tuméfaction faciale), signant généralement une extension locorégionale importante, qui attirent l'attention. Cela explique la grande latence avant le diagnostic : plus de 6 mois pour la majorité de nos patients.

Pour cette raison, il existe une proportion élevée de stades avancés au moment du diagnostic. Tel est le cas dans notre étude puisque près de $60 \%$ des lésions étaient initialement classées « $T_{3} / T_{4} »$.

Dans la majorité des séries, ces tumeurs atteignent préférentiellement les sinus ethmoïdaux, notamment lorsqu'une exposition aux poussières organiques de bois durs ou de cuirs étaient retrouvée (particules $>5 \mu$ ). Ce fait était également noté dans notre étude où près de $80 \%$ des sujets exposés à un facteur de risque développaient une lésion tumorale ethmoïdale.

Les extensions ganglionnaires sont peu fréquentes, touchant seulement 2 patients $(5,3 \%)$ dans notre étude. En fonction des auteurs, l'atteinte ganglionnaire varie de 1 à $26 \%$ [13]. De même seuls 6 patients (16\%) ont développé une métastase viscérale à distance. Là encore, ces données sont accord avec celles de la littérature, l'agressivité et le pronostic de ces lésions étant plutôt liés à leur extension locorégionale et au taux de récidive locale (estimé à 33,6\% dans notre étude, et à $40 \%-50 \%$ des cas de la littérature [121]).

Le délai moyen de récidive est de 28 mois [11]. Notre étude montre un délai quasiment identique, estimé à 30,25 mois. Les facteurs de mauvais pronostic reconnus sont : la non exposition aux poussières de bois, l'envahissement endocrânien, du sphénoïde ou du nasopharynx, la présence d'adénopathie, des marges chirurgicales non saines ainsi que le stade $T_{4}$ et principalement le stade $T_{4 b}$. 
Notre étude souligne l'impact pronostic de la classification TNM : la majorité des tumeurs initialement classé « $T_{4}$ » a montré une évolution défavorable.

En effet, $66 \%$ des lésions ayant récidivé localement et 100\% de celles ayant présenté des adénopathies métastatiques étaient " $T_{4}$ ». De même, $50 \%$ des patients présentant une tumeur « $T_{4}$ » sont décédés des suites de leur pathologie. De plus, notre analyse a mis en évidence un risque de décès quasiment 5 fois plus important chez les sujets dont la tumeur était classée " $T_{3}$ ou $T_{4}$ " par rapport aux sujets dont la tumeur était initialement « $T_{1}$ ou $T_{2}$ » $(p=0,02)$. Cependant, autant de lésions initialement classées " $T_{2}$ » et « $T_{4}$ » ont présenté une métastase viscérale à distance.

La survie globale des tumeurs de l'ethmoïde est passée de $28 \% \pm 13 \%$ dans les années 1960 à $51 \% \pm 14 \%$ dans les années 1990 . Actuellement elle fluctue autour de $60 \%$. Notre série montre une survie globale à 3 ans superposable, estimée à $56 \%$.

Le bilan radiologique pré-thérapeutique des adénocarcinomes nasosinusiens comporte actuellement de manière systématique un scanner et une IRM du massif facial [31-33]. En effet, l'association de ces deux examens permet de réaliser une cartographie tumorale précise qui guidera l'attitude thérapeutique. L'IRM, associée au scanner offre une corrélation histo-radiologique de $98,5 \%$, qui n'est que de $85,2 \%$ pour le scanner seul et de $94,1 \%$ pour l'IRM seule [13].

L'attitude thérapeutique vis-à-vis de ces lésions est maintenant codifiée, basée sur l'association radio-chirurgicale, rapportée dans $78,5 \%$ des études, améliorant le contrôle local ainsi que la survie globale. La quasi-totalité des patients de notre série a également bénéficié, sans distinction, de ce protocole thérapeutique (chirurgie + radiothérapie postopératoire systématique). 


\section{II - Analyse des caractéristiques morphologiques}

La répartition des différentes sous-variétés histologiques de notre série était :

- Identique à celle de la littérature en ce qui concerne le partage des sous-types « intestinal » versus «non intestinal », puisque $92 \%$ des patients présentaient une tumeur de type « intestinal », cette variété d'adénocarcinome étant la plus fréquemment rencontrée[22][51][69].

- Non comparable aux données des études pour la proportion des adénocarcinomes de type non intestinal de "bas grade " versus " haut grade ». En effet, parmi ces tumeurs, $67 \%$ étaient de " haut grade » et $33 \%$ de "bas grade ». Habituellement, l'effet inverse est observé, les "haut grades » étant considérés comme des tumeurs très rares[22][51][99-100].

- Non superposable aux résultats de la littérature en ce qui concerne chaque sous-groupe d'ITAC. Dans notre étude, bien que les "coliques" soient les plus représentés ( $37 \%$ des cas de notre série et $40 \%$ dans la littérature), les " mucineux » et "mixtes " sont bien plus fréquents dans notre étude que dans les différentes publications (48\% versus $22 \%$ dans la littérature) [22] [32].

Même si l'exposition aux poussières de bois et de cuirs est depuis longtemps considérée comme un facteur de risque dans la survenue des adénocarcinomes de l'ethmoïde, cette vérité ne concerne habituellement pas les tumeurs de type " non intestinal » [22][36][69]. Dans notre étude, contrairement aux ITAC où une exposition était retrouvée dans près de $86 \%$ des cas, seul un des trois cas d'adénocarcinomes de type non intestinal y a été exposé. 
Plusieurs publications montrent une corrélation entre le sous-type histologique et le pronostic [22][51][58].

Au sein des ITAC, les formes " non mucineuses » (« coliques », " papillaires » et " solides ») et cytologiquement bien différenciées auraient un meilleur pronostic que les formes « avec mucines » (« mucineux » et « mixte ») et peu différenciées. Nos résultats sont comparables à ces données, puisque la majorité des tumeurs classées initialement $\mathrm{T}_{4}$ et/ou ayant présenté une récidive locale ou des métastases ganglionnaires ou viscérales à distance était des adénocarcinomes de type « intestinal mucineux et/ou mixte ».

De même, $50 \%$ des patients atteints d'un ITAC « mucineux » et $42 \%$ atteints d'un ITAC « mixte » sont décédés (taux de survie à 3 ans de 46,3\%), contre $0 \%, 15 \%$ et $33 \%$ des patients atteints respectivement d'un ITAC « solide », « colique » ou « papillaire » (taux de survie à 3 ans de 61,4\%).

Cependant, la comparaison des résultats intéressant uniquement les sous-types « colique », " papillaire » et " solide », montre une légère différence avec ceux de la littérature. En effet, habituellement, le sous-type « papillaire » est de pronostic plus favorable [22], alors que dans notre étude, il semble plus péjoratif. Cela peut s'expliquer par le faible effectif de ce groupe dans notre étude (3 cas). II en est de même pour le sous-type « solide » $(2$ cas), habituellement plus agressif.

Pour les adénocarcinomes de type «non intestinal», les «bas grades » ont généralement un meilleur pronostic que les « hauts grades » [99-100]. Cela était également le cas dans notre série. En effet, lorsqu'une récidive locale, une lésion métastatique ganglionnaire ou viscérale voire même un décès, étaient observés dans ce sous groupe, cela touchait préférentiellement les sujets atteints d'un " haut grade $»$. 


\section{III - Analyse des caractéristiques immunohistochimiques}

II existe des différences phénotypiques entre les adénocarcinomes nasosinusiens de type « intestinal » et ceux de type « non intestinal » [60-69].

En effet, 94\% des cas d'ITAC de notre série exprimaient à la fois la CK20 et le CDX2. Dans $43 \%$ des cas, il s'y associe l'expression de la CK7, ce qui est compatible avec les différentes séries publiées.

Ainsi, la ressemblance histologique entre les adénocarcinomes nasosinusiens de type " intestinal » et les adénocarcinomes colorectaux ne se restreint pas à l'aspect morphologique, puisque ces types de tumeurs présentent des profils immunohistochimiques identiques « CK20 +, CK7 +/-, CDX $2+$ ».

Par ailleurs, il est intéressant de noter qu'à l'état normal, la muqueuse respiratoire n'exprime pas le CDX2, celui-ci étant caractéristique du phénotype intestinal.

$66 \%$ des cas d'adénocarcinomes de type " non intestinal » de notre étude montrent une expression de la CK7 alors qu'aucun d'entre eux n'exprimait la CK 20 ou le CDX2.

Ainsi, si le problème du diagnostic différentiel peut se poser entre les formes bien différenciées d'adénocarcinomes de type «intestinal » et les adénocarcinomes de type "non intestinal de bas grade », ces deux entités pouvant avoir un aspect histologique similaire, les anticorps CK7, CK20 et CDX2 constituent une aide importante au diagnostic. 
L'expression de la protéine EGFR au sein des ITAC est superposable à celle des adénocarcinomes colorectaux: $83 \%$ dans notre série et 75 à $90 \%$ selon les publications [69].

Dans une étude récente concernant 55 cas d'adénocarcinomes nasosinusiens, Franchi et al. [122] a évalué le taux d'expression de la protéine EGFR en immunohistochimie et les gains de copie du gène EGFR en FISH. Près d'un tiers des cas exprimaient la protéine EGFR et 7 d'entres eux présentaient également une augmentation du nombre de copie du gène, avec une corrélation significative entre ces données et la notion d'une exposition au bois. Cela n'a pas été retrouvé dans notre série $(p=0,3)$.

Pérez-Escuredo et al. [121] a également retrouvé une expression de la protéine EGFR en immunohistochimie sur une lignée cellulaire immortelle dérivée d'un ITAC de type « colique » (lignée ITAC-3).

L'étude de l'expression de la protéine EGFR par les cellules tumorales, en immunohistochimie, pourrait constituer un rationnel pour des essais thérapeutiques par thérapies ciblées de type anticorps monoclonaux "anti-EGFR » dans le traitement des ITAC, sachant que bon nombre de patients se retrouve en impasse thérapeutique et qu'aucune chimiothérapie n'a réellement fait ses preuves.

Cependant, au niveau des adénocarcinomes colorectaux, l'évaluation d'une possible corrélation entre l'expression immunohistochimique d'EGFR et la réponse thérapeutique s'est avérée rapidement décevante : en effet, des patients n'exprimant pas EGFR au niveau de leur tumeur selon les recommandations en vigueur (moins de $1 \%$ de cellules marquées) pouvaient néanmoins répondre au traitement [111][115] . 


\section{IV - Analyse des caractéristiques moléculaires}

De récentes études se sont intéressées à la détermination des altérations génétiques et aux voies potentiellement impliquées dans la genèse des adénocarcinomes nasosinusiens, afin de contribuer au développement de nouveaux marqueurs moléculaires pronostics et de stratégies thérapeutiques plus performantes.

Les mutations du gène KRAS ont été retrouvées de façon inhomogène au sein des différentes séries d'ITAC et sont généralement peu fréquentes ( 0 à $15 \%$ de mutations selon les études) $[83-87 ; 89]$. Néanmoins, une publication a rapporté un taux mutationnel de 50\% [88], quasi-similaire à celui retrouvé au niveau des adénocarcinomes colorectaux (35 à 40\%) [111][115].

Dans notre série, le taux de mutations ponctuelles du gène KRAS était faible, mais comparable aux données de la littérature, estimé à 13\%. Elles n'intéressaient que des sujets de sexe masculin, atteints d'ITAC et dont $80 \%$ avaient été exposés à un facteur de risque. Cependant, aucune corrélation significative n'a été retrouvée entre le statut mutationnel et les données épidémiologiques, cliniques ou histologiques.

Au sein des publications concernant des séries d'ITAC, les mutations KRAS de type « $G>A$ » étaient les plus fréquentes [50][84]. Cela était également le cas dans notre étude, puisque $60 \%$ des mutations étaient : c.35G>A ; p.G12D. De même, au niveau des adénocarcinomes colorectaux, les mutations les plus fréquemment rencontrées sont de type c.35G>A (38\% des cas).

Les discordances mutationnelles étaient de $66 \%$. L'apparition ou le changement mutationnel entre la tumeur primitive et la récidive locale ou à distance soulèvent des questions sur la nature polyclonale ou hétérogène de ces tumeurs. 
Notre étude n'avait retrouvé aucune altération du gène EGFR par technique SNaPshot $\circledast$ et analyse d'amplification de fragments.

Dans la littérature, il n'a été retrouvée aucune autre analyse comparable. En effet, les rares équipes s'étant intéressées à l'EGFR avaient plutôt réalisé des techniques de FISH ou d'immunohistochimie [122].

Bien qu'innovante, notre méthode d'étude de l'EGFR n'est pas exhaustive, ne ciblant que les altérations les plus fréquemment décrites pour induire une sensibilité ou une résistance aux « nib TKI » (inhibiteurs du domaine tyrosine kinase) chez des sujets atteints de carcinomes broncho-pulmonaires non à petite cellule. Un séquençage complet du domaine tyrosine kinase de l'EGFR pourrait s'avérer intéressant, pour détecter des mutations rares ou non décrites.

Par ailleurs, les techniques de détection des anomalies génétiques utilisées dans notre étude présentent certaines limites. En effet, un pourcentage de $20 \%$ de cellules tumorales est normalement requis pour valider un résultat de biologie moléculaire. II apparaît donc important de déterminer le pourcentage de cellules tumorales sur la surface de prélèvement examiné, car la quantité d'ADN extraite y sera différente en fonction de ces paramètres.

De plus, de nombreux prélèvements présentaient des remaniements nécrotiques et/ou un important stroma mucineux, ces deux phénomènes pouvant altérer de façon considérable la qualité de l'ADN extrait.

En fonction du type de fixateur utilisé, de l'utilisation préalable d'agents décalcifiant et de la qualité de la conservation des blocs de paraffine, ces techniques peuvent s'avérer négatives. Des mutations artéfactuelles ont également été décrites pour des prélèvements fixés au formol et inclus en paraffine[123]. De ce fait, deux analyses indépendantes doivent être réalisées avant de conclure. 
Plus récemment, des études du génome entier par CGH-array ( Comparative Genomic Hybridization ») [50][124] ont également été réalisées sur des séries d'ITAC et ont révélé de fréquents gains de copie au niveau des loci 7q11-21 (71\% des cas), $18 p 11-2$ (66\% des cas), 8q11-22 (62\% des cas), 5p11-13 (57\% des cas), 12q11-13 et $19 p$ ( $52 \%$ des cas). Des pertes de copie étaient identifiées au niveau des loci 8p22-23, 18q22-23, 17p13 et 5q31-qter. Ces anomalies chromosomiques étaient très différentes de celles retrouvées au niveau des autres tumeurs malignes de la région " tête et cou», mais étaient parfois similaires à celles des adénocarcinomes colorectaux [50]. Ces études ont montré que certains loci sièges de gains de copie, codaient pour des facteurs de croissance, tels que HGF (« hepatocyte growth factor »), en 7q21-22 et MOS ou C-MYC en 8q. De même, des loci sièges de pertes de copies intéressaient des gènes suppresseurs de tumeurs, tels que $D C C$ et SMAD4 au locus 18q, APC en $5 q$ et TP53 en 17p13. Par ailleurs, un plus grand nombre de gains de copie était retrouvé au niveau des adénocarcinomes peu différenciés, ce fait indiquant une probable participation du phénomène d'amplification génique dans le processus de perte de différenciation des ITAC.

Une étude par « CGH-microarray » a été réalisée sur une série de patient présentant un ITAC et une exposition au bois [77]. Les gains de copie les plus fréquents étaient localisés en $5 p 15,20 q 13$ et $8 q 24$, alors que les pertes survenaient plus fréquemment en 4q31-qter, 18q12-22, 8p12-22, 8p12-pter et 5q11-qter.

Encore une fois, des similitudes entre ces altérations et celles notées au niveau des adénocarcinomes colorectaux étaient retrouvées [50].

Bien qu'aucune corrélation significative n'ait été mise en évidence entre ces données et les paramètres cliniques (notamment l'exposition professionnelle aux poussières organiques de bois ou de cuir), la découverte de ces altérations permettrait de mieux comprendre la pathogenèse des ITAC et de mettre en évidence de nouveaux « marqueurs moléculaires » de diagnostic précoce de ce type de lésion chez des sujets exposés. 


\section{CONCLUSION}

Cette étude réalisée sur 38 cas d'adénocarcinomes des fosses nasales et des sinus para-nasaux et qui constitue l'expérience du CHU de Rouen pour cette pathologie a permis de faire le point sur cette entité de tumeurs rares.

Les données épidémiologiques, cliniques, thérapeutiques et morphologiques sont sensiblement comparables à celles de la littérature.

Le diagnostic de cette pathologie est encore fait le plus souvent à un stade tumoral élevé, en raison du caractère aspécifique et tardif de la symptomatologie fonctionnelle.

II apparaît important de comprendre les voies de tumorogenèse de ces lésions afin de déterminer quels marqueurs morphologiques ou moléculaires pourraient être considérés comme « à risque » de développer de telles tumeurs.

Notre travail a permis de confirmer que le phénotype et le génotype des adénocarcinomes nasosinusiens de type " intestinal » étaient quasi similaires à ceux des adénocarcinomes colorectaux, renforçant ainsi les hypothèses anciennes de l'oncogenèse de ces tumeurs.

Cette parenté suggère également de proposer, pour les cas non chirurgicaux, une approche thérapeutique identique à celle utilisée pour le traitement des carcinomes colorectaux métastatiques ; ceci devra être évalué par un essai clinique. 
De ce fait, chaque cas d'adénocarcinome nasosinusien devrait bénéficier d'une étude immunohistochimique incluant au minimum la cytokératine 20 et/ou le CDX2. Une positivité de ces immunomarquages orienterait plutôt vers une tumeur de type « intestinal ».

Dans le cas des lésions évoluées, inopérables, une recherche en biologie moléculaire de mutations du gène KRAS pourrait être effectuée, afin de proposer aux patients ne présentant pas de mutation de ce gène, une thérapie ciblée par des anticorps monoclonaux "anti-EGFR», qui ont prouvé leur efficacité contre les adénocarcinomes colorectaux métastatiques.

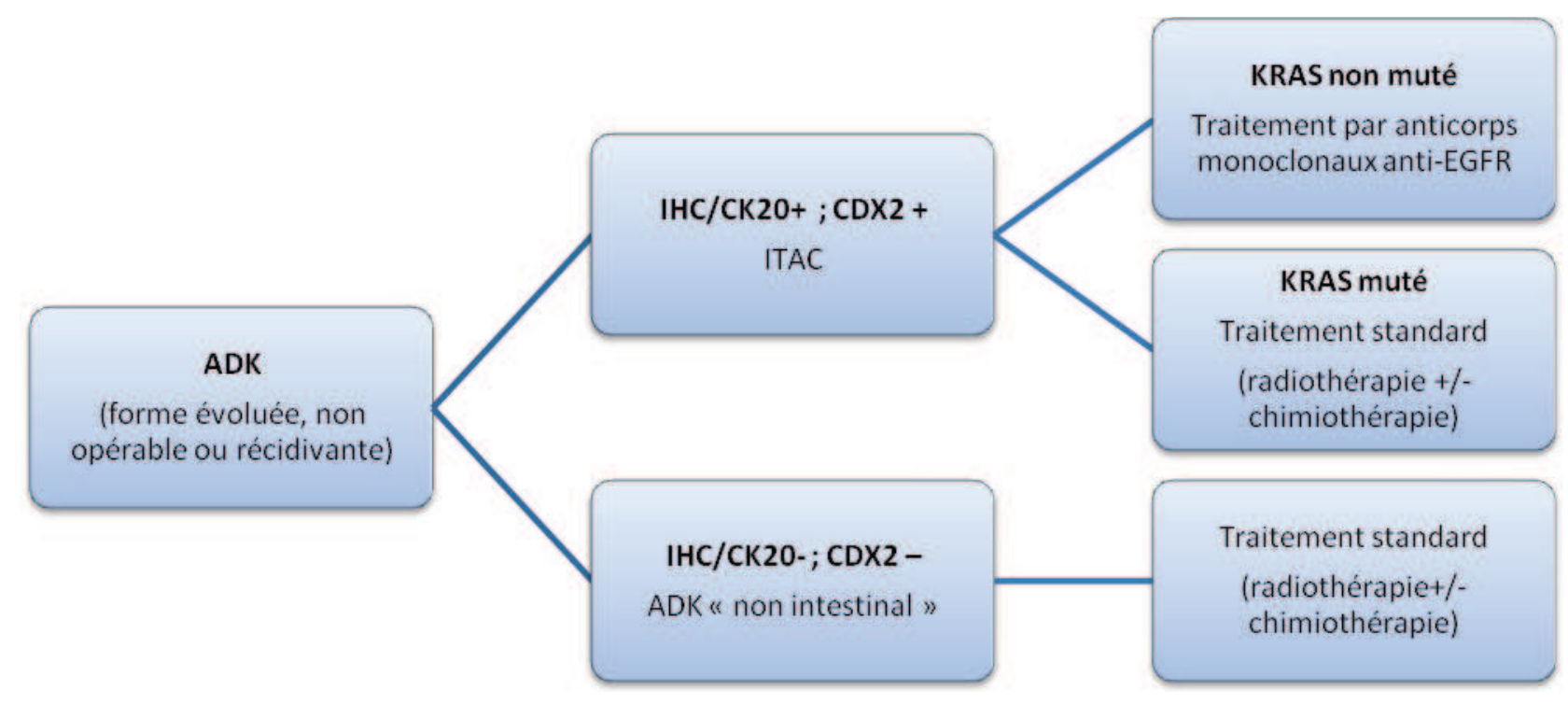

Figure 42 :

Proposition d'une stratégie diagnostique et thérapeutique pour les adénocarcinomes nasosinusiens. 


\section{BIBLIOGRAPHIE}

[1] Champsaur P, Pascal T, Vidal V, Gaubert JY, Bartoli JM, Moulin G. Imaging anatomy of the paranasal sinuses. J. Radiol. 2003 (84) : 885-900.

[2] Netter FH. Atlas Of Human Anatomy. Switzerland, CIBA-CEIGY Corporation. 1991.

[3] Fawcett W, Jensh RP. Histologie, l'essentiel. Paris. Maloine. 2002.

[4] Homer JJ, Jones NS, Bradley PJ. The role of endoscopy in the management of nasal neoplasia. Am J Rhinol. 1997 (11) : 41-47.

[5] Bhattacharyya N. Factors predicting survival for cancer of the ethmoid sinus. Am J Rhinol. 2002 (16) : 281-286.

[6] Muir C, Weiland L. Upper aerodigestive tract cancers. Cancer. 1995 (75) : 147-153.

[7] Brasnu D, Roux FX. Cancer of the ethmoid sinus. Rev Prat. 2000 (50) : 15621567.

[8] Curado MP, Edwards B, Shin HR, Storm H, Ferlay J, Heannue M. Cancer Incidence in Five Continents, 2007 Vol IX. IARC Scientific Publications No.160.Lyon: IARC (International Agency for Research on Cancer).

[9] Breheret R, Laccourreye L, Jeufroy C, Bizon A. Adenocarcinoma of the ethmoid sinus : retrospective study of 42 cases. Eur Ann Otorhinolaryngol Head Neck Dis. 2011 (128) : 211-217.

[10] De Gabory L, Manoury A, Maurice-Tison S, Merza Abdulkhaleq H, Darrouzet $V$, Bébéar JP, Stoll D. Long term single-center results of management of ethoid adenocarcinoma : 95 patients over 28 years. Ann Surg Oncol. 2010 (17) : 1127-1134.

[11] Choussy O, Ferron C, Vedrine PO, Toussaint B, Liétin B, Marandas P, Babin E, De Raucourt D, Reyt E, Cosmidis A, Markeiff M, Dehesdin D. Adenocarcinoma of ethmoid : a GETTEC retrospective multicenter study of 418 cases. Laryngoscope 2008 (118) : 437-443.

[12] Fontana L, Liétin B, Catilina P, Devif C, Féneon B, Martin F, Mom T, Gilain L. Occupational exposure to wood dust and nasal sinus cancer. Ann Otolaryngol Chir Cervicofac. 2008 (125) : 65-71.

[13] Percodani J, Rose X, Folia M, Woisard V, Uro-Coste E, Pessey JJ, Serrano E. Les adénocarcinomes de l'ethmoïde : à propos de 46 patients. Fr ORL. 2005 (89) : 146-154.

[14] Liétin B, Mom T, Avan P, Llompart X, Kemeny JL, Chazal J, Russier M, Gilain L. Adenocarcinomas of the ethmoid sinus : retrospective analysis of prognostics factors. Ann Otolaryngol Chir Cervicofac. 2006 (123) : 211-220. 
[15] Jegoux F, Ferron C, Malard O, Cariou G, Faure A, Beauvillain de Montreuil C. Ethmoid adenocarcinoma: transfacial approach for anterior skull base resection. A series of 80 cases. Ann Otolaryngol Chir Cervicofac. 2004 (121) : 213-221.

[16] Michel L, Ayache S, Guillaume-Souaid G, Toussaint P, Le Gars D, Deschepper B, Chatelain D, Gontier-Calvet MF, Strunski V. Combined transfacial and neurosurgical approach for the treatment oh ethmoid cancers. Ann Otolaryngol Chir Cervicofac. 2006 (123) : 21-29.

[17] Guillote-Van Gorkum ML, Nasser T, Mérol JC, Legros M, Rousseaux P, Chays A. Ethmoid adenocarcinoma : a series of 17 cases. Ann Otolaryngol Chir Cervicofac. 2006 (120) : 296-301.

[18] Claus F, Boterberg T, Ost P, Huys J, Vermeersch H, Braems S, Bonte K, Moerman M, Verhoye C, De Neve W. Postoperative radiotherapy for adenocarcinoma of the ethmoid sinuses : treatment results for 47 patients. Int $\mathrm{J}$ Radiat Oncol Biol Phys. 2002 (54):1089-1094.

[19] Dulguerov P, Jacobsen MS, Allal AS, Lehmann W, Calcaterra T. Nasal and paranasal sinus carcinoma : are we making progress? A series of 220 patients and a systematic review. Cancer. 2001 (92) : 3012-3029.

[20] Choussy O, Lerosey Y, Marie JP, Dhermain F, Seng SH, Francois A, AndrieuGuitancourt J, Dehesdin D. Adenocarcinoma of the ethmoid sinuses : results of a retrospective study in Rouen. Ann Otolaryngol Chir Cervicofac. 2001 (118) : 156-164.

[21] Stoll D, Bébéar JP, Truilhé $Y$, Darrouzet V, David N. Ethmoid adenocarcinomas : retrospective study of 76 patients. Rev Laryngol Otol Rhinol. 2001 (122) : 21-29.

[22] Franchi A, Santucci M, Wenig B. Adenocarcinoma. In : Barnes L, Eveson JW, Reichart P, Sidransky D. Pathology and genetics head and neck tumours. World Health Organisation classification of tumours. 3th edition. Lyon. IARC 2005.

[23] Waldron JN, O'Sullivan B, Warde P, Gullane P, Lui FF, Payne D, Cummings B. Ethmoid sinus cancer: twenty-nine cases managed with primary radiation therapy. Int J Radiat Oncol Biol Phys. 1998 (41) : 361-369.

[24] Schwaab, G., M. Julieron, and F. Janot, Epidemiology of cancers of the nasal cavities and paranasal sinuses. Neurochirurgie. 1997 (43) : 61-63.

[25] Luce D, Bégin D, Demers PA, Gérin M, Orlowski E, Kogevinas M, Belli S, Bugel I, Bolm-Audorff U, Brinton LA, Comba P, Hardell L, Hayes RB, Magnani C, Merler E, Preston-Martin S, Vaughan TL, Zheng W, Boffetta P. Sinonasal cancer and occupational exposures: a pooled analysis of 12 case-control studies. Cancer Causes Control.2002 (13) : 147-157.

[26] Debry C, Corniaux C, Budor P, Tumeurs malignes de l'ethmoïde. Signes révélateurs. JFORL. 1989 (38): 3-9. 
[27] Kraus DH, Sterman BM, Levine HL, Wood BG, Tucker HM, Lavertu P. Factors influencing survival in ethmoid sinus cancer. Arch Otolaryngol Head Neck Surg. 1992 (118) : 367-372.

[28] Lund VJ and Zanation A. European position paper on endoscopic management of tumours of the nose, paranasal sinuses and skull base. Rhinol Suppl. 2010 (22) : $1-143$.

[29] Civit T, Pinelli C, Freppel S, Baylac F. Orbital tumors arising from paranasal sinuses. Neurochirurgie. 2010 (56) : 174-182.

[30] Suárez C, Llorente JL, Fernández de León R, Cabanillas R, Suárez V, López A. Anterior craniofacial resection: oncologic outcome and complications in a series of 111 cases. Acta Otorrinolaringol Esp. 2004 (55) : 27-33.

[31] Lund VJ, Howard DJ, Llyod GA, Cheesman AD. Magnetic resonance imaging of paranasal sinus tumors for craniofacial resection. Head Neck. 1989 (11) : 279-283.

[32] Elkeslassy, A, Meder JF, Laffite F, Rezeai K, Fredy D. Imaging of non-osseous malignant tumors of the anterior skull base. Preoperative evaluation. Neurochirurgie, 1997 (43): 68-75.

[33] Kondo M, Horiuchi M, Shiga H, Inuyama Y, Dokiya T, Takata Y, Yamashita S, Ido $\mathrm{K}$, Ando $\mathrm{Y}$, Iwata $\mathrm{Y}$, Hashimoto S. Computed tomography of malignant tumors of the nasal cavity and paranasal sinuses. Cancer, 1982 (50): 226-231.

[34] Sobin LH, Wittekind CH. International Union Against Cancer. Paranasal sinus. In : TNM classification of malignant tumours, $7^{\text {th }}$ ed. New York. John Wiley and Sons Inc. 2010.

[35] Sobin $\mathrm{LH}$, Wittekind $\mathrm{CH}$. International Union Against Cancer. Paranasal sinus. In : TNM classification of malignant tumours, $5^{\text {th }}$ ed. New York. John Wiley and Sons Inc. 1997.

[36] Mills S, Gaffey M, Frierson H. Atlas of tumor pathology. Tumor of the upper aerodigestive tract and ear. $3^{\text {th }}$ ed. Armed Force International Pathology Tumors of the Upper Aerodigestive Tract and Ear. 2000 : 107-116.

[37] Macbeth R. Malignant disease of the paranasal sinuses. J Laryngol Otol. 1965 (79) : 592-612.

[38] Acheson ED, Hadfield EH, Macbeth R. Carcinoma of the nasal cavity and accessory sinuses in woodworkers. Lancet. 1967 (7485): 311-312.

[39] Llorente JL, Aldama P, Alvarez-Marcos C, Escuredo J, Alonso-Guervos M, Fresno F, Suarez C, Hermsen M. Nasosinusal Adenocarcinoma : Molecular and Genetic Analysis by MLPA. Acta Otorrinolaringol Esp. 2008 (59) : 151-158.

[40] Llorente JL, Escuredo J, Alvarez-Marcos C, Suarez C, Hermsen M. Genetic and clinical aspects of wood dust related intestinal-type sinonasal adenocarcinoma : a review. Eur Arch Otorhinolaryngol. 2009 (266) :1-7. 
[41] IARC Monographs on the evaluation of carcinogenic risks to humans. Volume 25 : wood, leather and some associated industries. Lyon : IARC. 1981.

[42] IARC Monographs on the evaluation of carcinogenic risks to humans. Spplement No.7 : overall evaluations of carcinogenicity : an updating of IARC Monographs Volumes 1 to 42. Lyon : IARC.1987.

[43] Merler E, Baldasseroni A, Laria R, Faravelli P, Agostini R, Pisa R, Berrino F. On the causal association between exposure to leather dust and nasal cancer : further evidence from a case-control study. Br J Ind Med. 1986 (43) : 91-95.

[44] Mikoczy Z, Hagmar L. Cancer incidence in the Swedish leather tanning industry : updated findings 1958-99. Occup Environ Med. 2005 (62) : 461-464.

[45] Bonneterre V, Deschamps E, Persoons R, Bernardet C, Liaudy S, Maitre A, de Gaudemaris R. Sinonasal cancer and exposure to leather dust. Occup Med (Lond). 2007 (57) : 438-443.

[46] Iaia TE, Farina G, Ferraro MT, Miceli GB, Mugnaini E, Papaleo B, Battista G. A series of cases of sinonasal cancer among shoemakers and tannery workers. Epidemiol Prev. 2004 (28) : 169-173.

[47] Korpassy B, Mosonyi M. The carcinogenic activity of tannic acid; liver tumours induced in rats by prolonged subcutaneous administration of tannic acid solutions. Br J Cancer. 1950 (4) : 411-420.

[48] Barton RT. Nickel carcinogenesis of the respiratory tract. J Otolaryngol 1977 (5) :412-422.

[49] Luce D, Gerin M, Leclerc A, Morcet JF, Brugere J, Goldberg M. Sinonasal cancer and occupational exposure to formaldehyde and other substances. Int $\mathrm{J}$ Cancer 1993 (53) : 224-231.

[50] Franchi A, Miligi A, Palomba A, Giovanneti L, Santucci M. Sinonasal carcinomas : recent advances in molecular and phenotypic characterization and their clinical implications. Crit Rev Oncol Hematol. 2011 (79) : 265-277.

[51] Poizat F, Maran-Gonzalez, Raynaud P, Baldet P, Garrel R, Crampette L, Costes V. Adenocarcinomas of nasal cavities and paranasal sinuses : diagnostic pitfalls in sinonasal glandular lesions. Ann Pathol. 2009 (29) : 286295.

[52] Kleinsasser O, Schroeder HG. Adenocarcinomas of the inner nose after exposure to wood dust: morphological findings and relationships between histology and clinical behaviour in 79 cases. Arch Otorhinolaryngol 1988 (245) : $1-15$.

[53] Barnes L. Intestinal-type adenocarcinoma of the nasal cavity and paranasal sinuses. Am J Surg Pathol 1986 (10) : 192-202.

[54] Batsakis JG, Holtz F, Sueper RH. Adenocarcinoma of nasal and paranasal cavities. Arch Otorhinolaryngol 1963 (77) : 625-33. 
[55] Gamez-Araujo JJ, Ayala AG, Guillamondegui O. Mucinous adenocarcinomas of the nose and paranasal sinuses. Cancer 1975 (36) : 1100-1105.

[56] Klintenberg C, Olofosson J, Hellquist H, Sökjer H. Adenocarcinoma of the ethmoid sinuses : a review of 28 cases with special reference to wood dust exposure. Cancer 1984 (54) : 482-488.

[57] Franquemont DW, Fechner RE, Mills SE. Histologic classification of sinonasal intestinal type adenocarcinomas. Am J Surg Pathol 1991 (15) : 368-375.

[58] Franchi A, Gallo O, Santucci M. Clinical relevance of the histological classification of sinonasal intestinal-type adenocarcinomas. Hum Path 1999 (30) : 1140-1145.

[59] Batsakis JG, Mackay B, Ordonez NG. Enteric type adenocarcinoma of the nasal cavity. An electron microscopic and immunocytochemical study. Cancer. 1984 (54) : 855-860.

[60] Mac Kinney CD, Mills SE, Franquemont DW. Sinonasal intestinal-type adenocarcinoma: immunohistochemical profil and comparaison with colonic adenocarcinoma. Mod Pathol 1995 (8) : 421-426.

[61] Krane JF, O'Connel JT, Plich BZ, et al. Cytokeratin and mucin expression in intestinal-type adenocarcinoma. Mod Pathol 2000 (13) : 139A.

[62] Bashir AA, Robinson RA, Benda JA, Smith RB. Sinonasal adenocarcinoma: immunohistochemical marking and expression of oncoproteins. Head and Neck 2003 (25) : 763-771.

[63] Choi HR, Sturgis E, Rashid A, Demonte F, Luna M, Batsakis J, El Naggar AK. Sinonasal adenocarcinoma: evidence of histologic divergence of enteric and nonenteric phenotypes. Hum Path 2003 (34) : 1101-1107.

[64] Abecassis J, Viana G, Pissarra C, Pereira T, Fonseca I, Soares J. Adenocarcinomas of the nasal cavity and paranasal sinuses: a clinicopathological and immunohistochemical study of 14 cases. Histopathology 2004 (45) : 254-259.

[65] Kennedy M, Jordan RC, Berean KW, Perez-Ordonez B. Expression pattern of CK7, CK20, CDX2 and villin in intestinal-type sinonasal adenocarcinoma. J Clin Pathol 2004 (57) :932-937.

[66] Franchi A, Massi D, Palomba A, Biancalani M, Santucci M. CDX-2, cytokeratin 7 and cytokeratin 20 immunohistochemical expression in the diagnosis of primary adenocarcinomas of the sinonasal tract. Virchows Arch 2004 (445) : 63-67. 
[67] Cathro HP, Mills SE. Immunophenotypic differences between intestinal-type and low-grade papillary sinonasal adenocarcinoma, An immunohistochemical study of 22 cases utilizing CDX2 and MUC2. Am J Surg Pathol 2004 (28) : 1026-1032.

[68] Castillo C, Buob D, Mortuaire G, Chevalier D, Aubert S, Copin MC, Leroy X. Signet-ring cell adenocarcinoma of sinonasal tract. An immunohistochemical study of the mucins profile. Arch Pathol Lab Med. 2007 (131) : 961-964.

[69] El Ayoubi A, Poizat F, Garrel R, Costes V, Guerrier B, Essakalli, Kzadri M, Crampette L. Sinonasal adenocarcinomas reviewed. Prognostic value of WHO 2005 histological classification. Ann Otolaryngol Chir Cervicofac. 2009 (126) : 175-181.

[70] Järvi A. Heterotopic tumors with an intestinal mucous membrane structure in the nasal cavity. Acta Otolaryngol. 1945 (33) : 471-485.

[71] Mills SE, Fechner RE, Cantrell RW. Aggressive sinonasal lesion resembling normal intestinal mucosae. Am J Surg Pathol. 1982 (6) : 803-809.

[72] Wilhelmsson B, Hellquist $\mathrm{H}$, Olofsson J, Klintenberg C. Nasal cuboidal metaplasia with displasia. Precursor to adenocarcinoma in wood-dust- exposed workers? Acta Otolaryngol. 1985 (99) : 641-648.

[73] Wilhelmsson B, Lundh B. Nasal epithelium in woodworkers in the furniture industry. A histological and cytological study. Acta Otolaryngol. 1984 (98) : 321-324.

[74] Boysen M, Voss R, Solberg LA. The nasal mucosae in softwood exposed furniture workers. Acta Otolaryngol. 1986 (101) : 501-508.

[75] Palomba A, laia TE, Biancalani M, Conti S, Battista G, Papaleo B, Franchi A. A morphologic and immunohistochemical study of nasal mucosae in leatherworkers. Am J Rhinol. 2008 (22) : 356-360.

[76] Vivanco B, Llorente JL, Escuredo J, Alvarez-Marcos C, Fresno MF, Hermsen M. Benign lesions in mucosae adjacent to intestinal type sinonasal adenocarcinoma. Patholog Res Int. 2011 (20) : 1-8.

[77] Hermsen MA, Lliorente JL, Perez-Escudero J, Lopez F, Ylstra B, AlvaresMarcos C. Genome-wide analysis of genetic changes in intestinal-type adenocarcinoma. Head Neck 2009 (31) : 290-297.

[78] Holmila R, Cyr D, Luce D, Heikkilä $P$, Dictor $M$, Steiniche T, Stjernvall T, Bornholdt J, Wallin H, Wolff $H$, Husgafvel-Pursiainen K. COX-2 and p53 in human sinonasal cancer : COX-2 expression is associated with adenocarcinoma histology and wood-dust exposure. Int J Cancer. 2008 (122) : 2154-2159. 
[79] Holmila R, Bornholdt J, Heikkilä P, Suitiala T, Févotte J, Cyr D, Hansen J, Snellman SM, Dictor M, Steiniche T, Schlünssen V, Schneider T, Pukkala E, Savolainen K, Wolff H, Wallin H, Luce D, Husgafvel-Pursiainen K. Mutations in TP53 tumor suppressor gene in wood duqt-related sinonasal cancer. Int $\mathrm{J}$ Cancer. 2010 (127) : 578-588.

[80] Holmila R, Bornholdt J, Suitiala T, Cyr D, Dictor M, Steiniche T, Wolff H, Wallin $H$, Luce D, Husgafvel-Pursiainen K. Profile of TP53 gene mutations in sinonasal cancer. Mutat Res. 2010 (686) : 9-14.

[81] Valente G, Ferrari L, Kerim S, Gervasio CF, Ricci E, Migliaretti G, Pira E, Bussi M. Evidence of p53 immunohistochemical overexpression in ethmoidal mucosae of woodworkers. Cancer Detect Prev. 2004 (28) : 99-106.

[82] Palomba A, laia T, Biancalani M, Franchi A. p53 expression in sinonasal mucosae of leatherworkers. Virchows Arch. 2007 : 451-462.

[83] Wu TT, Barnes L, Bakker A, Swalsky PA, Finkelstein SD. Kras-2 and p53 genotyping of intestinal-type adenocarcinoma of the nasal cavity and the paranasal sinuses. Mod Pathol. 1996 (9) : 199-204.

[84] Saber AT, Nielsen LR, Dictor M, Hagmar L, Mikoczy Z, Wallin H. K-ras mutations in sinonasal adenocarcinomas in patients occupationally exposed to wood or leather dust. Cancer letters. 1998 (126) : 59-65.

[85] Perez P, Domingez O, Gonzalez S, Gonzalez S, Trivino A, Suarez C. Ras gene mutations in ethmoid sinus adenocarcinoma: prognostic implications. Cancer. 1999 (86) : 225-264.

[86] Yom SS, Rashid A, Rosenthal DI, Elliot DD, Hanna EY, Weber RS, El-Naggar AK. Genetic analysis of sinonasal adenocarcinoma phenotype: distinct alterations of histologic significance. Modern Path. 2005 (18) : 315-319.

[87] Perez-Ordonez B, Huynh N, Berean KW, Jordan RC. Expression of mismatch repair proteins, $B$ Catenin, and $E$ cadherin in intestinal-type sinonasal adenocarcinoma. J Clin Path. 2004 (57) : 1080-1083.

[88] Frattini M, Perrone F, Suardi S, Balestra D, Caramuta S, Colombo F, Licitra L, Cantu G, Pierotti MA, Pilotti S. Phenotype-genotype correlation: challenge of intestinal-type adenocarcinoma of the nasal cavity and paranasal sinuses. Head Neck. 2006 (28) : 909-915.

[89] Bornholdt J, Hansen J, Steiniche T, Dictor M, Antonsen A, Wolff H,Schlünssen V, Holmila R, Luce D, Vogel U, Husgavel-Pursiainen $K$, Wallin H. K-ras mutations in sinonasal cancers in relation to wood dust exposure. BMC cancer. $2008(20) ; 53: 1-11$.

[90] Martinez JG, Perez-Escudero J, Lopez F, Suarez C, Alvares- Marcos C, Llorente JL. Microsatellite instability analysis of sinonasal adenocarcinomas. Otolaryngol Head Neck Sur. 2009 (140) : 55-60. 
[91] Franchi A, Palomba A, Fondi C, Miligi L, Paglierani M, Pepi M, Santucci M. Immunohistochemical investigation of tumorigenic pathways in sinonasal intestinal-type adenocarcinoma. A tissue microarray analysis of 62 cases. Histopathology. 2011(59) : 98-105.

[92] Alvarez I, Suarez C, Rodrigo JP, Nunez F, Caminero MJ. Prognostic factors of paranasal sinus cancer. Am J Otolaryngol. 1995 (16) : 109-114.

[93] Orvidas LJ, Lewis JE, Weaver AL, Bagniewski SM, Olsen KD. Adenocarcinoma of the nose and paranasal sinuses: a retrospective study of diagnosis, histologic characteristics, outcome in 24 patients. Head and Neck. 2005 (27) : 370-375.

[94] Heffner D, Hyams V, Hauck K, Lingeman C. Low-grade adenocarcinoma of the nasal cavity and paranasal sinuses. Cancer 1982 (50) : 312-322.

[95] Kleinsasser O. Terminal tubulus adenocarcinoma of the nasal seromucous glands. A specific entity. Arch Otorhinolaryngol. 1985 (241) : 183-193.

[96] Neto A, Pineda-Daboin K, Luna M. Sinonasal tract seromucous adenocarcinomas: a report of 12 cases. Ann Diagn Pathol. 2003 (7) : 154-159.

[97] Skalova A, Cardesa A, Pfaltz M, Ryska A, Simpson R, Michak M. Sinonasal tubulopapillary low-grade adenocarcinoma. Histological, immunohistological and ultrastructural features of poorly recognised entity. Virchows Arch. 2003 (443) : 152-158.

[98] Luna M. Sinonasal tubulopapillary low-grade adenocarcinoma. A specific diagnosis or just another seromucous adenocarcinoma? Adv Anat Pathol. 2005 (12) : 109-115.

[99] Jo V, Mills S, Cathro H, Carlson D, Stelow E. Low-grade sinonasal adenocarcinomas. The association with and distinction from respiratory epithelial adenomatoid hamartomas and other glandular lesions. Am J Surg Pathol. 2009 (33) : 401-408.

[100] Stelow E, Jo V, Mills S, Carlson D. A histologic and immunohistochemical study describing the diversity of tumors classified as sinonasal high grade non intestinal adenocarcinomas. Am J Surg Pathol. 2011(35) : 971-980.

[101] Franchi A, Palomba A, Massi D, Biancalani M, Sardi I, Gallo O, Santucci M. Low grade salivary type tubulopapillary adenocarcinoma of the sinonasal tract. Histopathology. 2006 (48) : 881-884.

[102] Ozolec J, Hunt JL. Tumor suppressor gene alterations in respiratory epithelial adenomatoid hamartoma (REAH): comparison to sinonasal adenocarcinoma and inflamed sinonasal mucosa. Am J Surg Pathol. 2006 (30) :1576-1580. 
[103] Ozolek J, Barnes L, Hunt J. Basal/myoepithelial cells in chronic sinusitis, respiratory epithelial adenomatoid hamartoma, inverted papilloma, and intestinal-type and non intestinal-type sinonasal adenocarcinoma. An immunohistochemical study. Arch Pathol Lab Med. 2007 (131) : 530-537.

[104] Perez-Ordonez B. Hamartomas, papillomas and adenocarcinomas of the sinonasal tract and nasopharynx. J Clin Pathol. 2009 (62) : 1085-1095.

[105] Saongoi AR, Berry G. Respiratory epithelial adenomatoid hamartoma : diagnostic pitfalls with emphasis on differential diagnosis. Adv Anat Pathol. 2007 (14) : 11-16.

[106] Weinreb I, Gnepp D, Laver N, Hoschar A, Hunt J, Seethala R, Barnes EL, Chetty R, Perez-Ordonez B. Seromucinous hamartomas : a clinicopathological study of sinonasal glandular lesion lacking myoepithelial cells. Histopathology. 2009 (54) : 205-213.

[107] Kim JY, Yoon JK, Citardi MJ, Batra PS, Roh HJ. The prevalence of human papilloma virus infection in sinonasal inverted papilloma specimens classified by histological grade. Am J Rhinol. 2007 (21) : 664-669.

[108] Neto AG, Pineda-Daboin K, Spencer ML, Luna MA. Sinonasal acinic cell carcinoma: a clinicopathologic study of four cases. Head Neck 2005 (27):603607.

[109] Berstein JM, Montgomery W, Balogh K. Metastatic tumor to the maxilla and paranasal sinuses. Laryngoscope. 1966 (76) : 621-650.

[110] Ruppert AM, Wislez M, Poulot V, Lacave R, Antoine M, Cadranel J. A simple view on lung cancer biology : the EGFR pathway. Rev Mal Respir. 2011 (28) : 565-577.

[111] Lamoril J, Ameziane N, Deybach JC, Bouizegarene P, Bogard M. KRAS and colorectal cancer : an important step to the personalized medicine. IBS. 2009 (24) : 196-209.

[112] Zandi R, Larsen AB, Andersen P, Stockhausen MT, Poulsen HS. Mechanisms for oncogenic activation of the epidermal growth factor receptor. Cell Signal. 2007 (19) : 2013-2023.

[113] Cortot AB. EGFR inhibitors in non-small cell lung cancer: more than yesterday (but less than tomorrow). Rev Pneumol Clin. 2010 (66) : 367-374.

[114] Sharma SV, Bell DW, Settleman J, Haber DA. Epidermal growth factor receptor mutations in lung cancer. Nat Rev Cancer. 2007 (7) : 169-181.

[115] Plesec TP, Hunt JL. KRAS mutation testing in colorectal cancer. Adv Anat Pathol. 2009 (16) : 196-203. 
[116] Suh E, Traber PG. An intestine-specific homeobox gene regulates proliferation and differentiation. Mol Cell Biol 1996 (16) : 619-625.

[117] Moskaluk CA, Zhang H, Powell SM, Cerilli LA, Hampton GM, Frierson HF Jr. $\mathrm{Cdx} 2$ protein expression in normal and malignant human tissues: an immunohistochemical survey using tissue microarrays. Mod Pathol 2003;16:913-9.

[118] Moll R, FrankeWW, Schiller DL, Geiger B, Krepler R. The catalog of human cytokeratins: Patterns of expression in normal epithelia, tumors, and cultured cells. Cell 1982 (31):11 - 24.

[119] Hernandez B, Frierson H, Moskaluk C, Li Y, Clegg L, Cote T, McCusker M, Hankey B, Edwards B, Goodman M. CK20 and CK7 protein expression in colorectal cancer : demonstration of the utility of a population-based tissue microarray. Hum Pathol 2005 (36) : 275-281.

[120] Tripodi D, Ferron C, Malard O, Beauvillain de Montreuil C, Planche L, SebilleRivain V, Roedlich C, Quemener S, Renaudin K, Longuenesse C, Verger C, Meflah K, Gratas C, Geraut C. Relevance of both individual risk factors and occupational exposure in cancer survival studies : the example of intestinal type sinonasal adenocarcinoma. Laryngoscope. 2011 (121) : 2011-2018.

[121] Perez-Escuredo J, Martinez J, Garcia-Inclan C, Vivanco B, Costales M, Alvarez-Marcas C, Llorente JL, Hermsen M. Establishment and genetic characterization of an immortal tumor cell line derived from intestinal-type sinonasal adenocarcinoma. Cell Onc. 2011 (34) : 23-31.

[122] Franchi A, Fondi C, Paglierani M, Pepi M, Gallo O, Santucci M. Epidermal growth factor receptor expression and gene copy number in sinonasal intestinal type adenocarcinoma. Oral Oncol. 2009 (45) : 835-838.

[123] Sabourin JC, Lamy A, Le Pessot F, Sesboüé R, Frébourg T. Pathologie moléculaire tumorale : à propos du génotypage de KRAS dans les adénocarcinomes colorectaux. Revue francophone des laboratoires. 2010 (418) : 43-46.

[124] Ariza M, Llorente JL, Alvarez-Marcas C, Baragano L, Salas A, RodriguezPrado N, Hermsen M, Suarez C, Sampedro A. Comparative genomic hybridization in primary sinonasal adenocarcinomas. Cancer. 2004 (100) : 335341. 
INTRODUCTION : Les adénocarcinomes primitifs des sinus et des fosses nasales sont des tumeurs rares, affectant tout particulièrement les travailleurs du bois. Généralement de révélation tardive, leur pronostic est souvent défavorable. La classification OMS 2005 en distingue deux types : " intestinal ou ITAC » et " non intestinal ». La parenté morphologique des ITAC avec les adénocarcinomes colorectaux fait évoquer la possibilité de mécanismes de carcinogenèse proches et des sensibilités thérapeutiques similaires, notamment aux nouvelles thérapies ciblées « anti-EGFR ».

OBJECTIFS : Caractériser le phénotype de ces tumeurs rares et évaluer l'expression de la protéine EGFR par des techniques immunohistochimiques et analyser leur génotype en recherchant la présence d'altérations somatiques des gènes EGFR et KRAS.

MATERIELS ET METHODES : Trente-huit cas d'adénocarcinomes nasosinusiens ont été recensés au CHU de Rouen entre 1986 et 2010. Pour chacun d'entre eux, les données épidémiologiques, les modalités de traitement et le suivi ont été étudiés. De même, une relecture anatomopathologique et une caractérisation phénotypique ont été effectuées, à l'aide de colorations standards et de techniques immunohistochimiques (cytokératines 7 et 20, CDX2 et EGFR). Le génotypage d'EGFR et de KRAS a été réalisé à la fois par SNaPshot multiplex® sur les produits d'amplifications de l'exon 2 (codons 12 et 13) de KRAS et des exons 18, 20 et 21 de l'EGFR mais aussi par analyse des fragments d'amplification des exons 19 et 20 de l'EGFR.

RESULTATS : Le sexe ratio était de 11,7H/1F, l'âge moyen au diagnostic de 64 ans. Dans la plupart des cas, une longue durée d'exposition au bois avait été retrouvée. Le principal signe fonctionnel était une obstruction nasale et la majorité des patients avaient bénéficié d'un traitement curatif chirurgical, souvent accompagné d'une radiothérapie. La classification TNM 2010 présentait un impact pronostic significatif, puisqu'un patient présentant une tumeur classée initialement « T3 ou T4 » avait quasiment 5 fois plus de risque de décéder de sa pathologie $(p=0,02)$. La majorité des sujets étaient atteints d'un adénocarcinome de type « intestinal » (CK20+,CDX2+,CK7+/-), 83\% d'entre eux exprimaient la protéine EGFR. Treize pour cent de mutations ponctuelles KRAS (60\% de type c.35G>A;p.G12D) et aucune mutation EGFR ont été identifiées. Néanmoins, il n'a pas été retrouvée de corrélation significative entre le statut mutationnel et les données épidémiologiques, cliniques ou histologiques. Notre travail confirme que le phénotype et le génotype des ITAC sont proches de ceux des adénocarcinomes colorectaux, renforçant ainsi les hypothèses anciennes de l'oncogenèse de ces tumeurs. Cette parenté suggère également de proposer pour les cas non chirurgicaux, une approche thérapeutique identique à celle utilisée pour le traitement des carcinomes colorectaux métastatiques ; ceci devra être validé par un essai clinique.

CONCLUSION : Chaque cas d'adénocarcinome nasosinusien devrait bénéficier d'une étude immunohistochimique incluant au minimum la cytokératine 20 et/ou le CDX2. Une positivité de ces immunomarquages orienterait plutôt vers une tumeur de type " intestinal ». Dans le cas des lésions évoluées, inopérables, une recherche en biologie moléculaire de mutations du gène KRAS devrait être effectuée, afin de proposer aux patients ne présentant pas de mutation de ce gène, une thérapie ciblée par des anticorps monoclonaux " antiEGFR », qui ont prouvé leur efficacité contre les adénocarcinomes colorectaux métastatiques. 
